REPRESENTATION THEORY

An Electronic Journal of the American Mathematical Society

Volume 10, Pages 314-352 (August 17, 2006)

S 1088-4165(06)00314-1

\title{
CHARACTER SHEAVES ON DISCONNECTED GROUPS, VIII
}

\author{
G. LUSZTIG
}

\begin{abstract}
In this paper we continue the study of character sheaves on a reductive group. To each subset of the set of simple reflections in the Weyl group we associate an algebra of the same kind as an Iwahori Hecke algebra with unequal parameters in terms of parabolic character sheaves. We also prove a Mackey type formula for character sheaves. We define a duality operation for character sheaves. We also prove a quasi-rationality property for character sheaves.
\end{abstract}

\section{INTRODUCTION}

Throughout this paper, $G$ denotes a fixed, not necessarily connected, reductive algebraic group over an algebraically closed field $\mathbf{k}$. This paper is a part of a series L9] which attempts to develop a theory of character sheaves on $G$.

In Section 36 we associate to any subset $J$ of the set of simple reflections an algebra $\underline{\mathfrak{K}}^{J}$ over $\mathbf{Q}(v)$ (with $v$ an indeterminate) defined using certain character sheaves on $Z_{J, G^{0}}$. When $J=\emptyset$, this is the standard Iwahori-Hecke algebra attached to $\mathbf{W}$. For general $J$ we show that this algebra shares several basic features with an Iwahori-Hecke algebra with unequal parameters. This opens the possibility of studying Iwahori-Hecke algebras with unequal parameters in the framework of the theory of perverse sheaves.

In Section 37 we prove a Mackey type formula for character sheaves on $Z_{J, D}$ where $J \subset \mathbf{I}$ and $D$ is a connected component of $G$. This is essentially an identity involving certain induction and restriction functors analogous to one in the representation theory of reductive groups over a finite field.

In Section 38 we study a duality operation for character sheaves on a connected component $D$ of $G$ generalizing the case $D=G^{0}$ considered in [L3, III, 15]. This duality operator is analogous to the known duality operator for representations of a reductive group over a finite field; see 38.12.

In Section 39 we prove a quasi-rationality property for representations of certain extensions of an (irreducible) Weyl group. This generalizes [L3, III, (12.9.3)] and is a step in the proof of a key property of character sheaves (see 39.8).

Notation. We write $\mathfrak{s}, \underline{\mathfrak{s}}$ instead of $\mathfrak{s}(\mathbf{T}), \underline{\mathfrak{s}}(\mathbf{T})$ (see 28.1, 28.3).

Errata to Part $V$. In 25.2 , line 1 , replace $\tilde{\mathcal{E}}^{\prime}$ by $\pi_{1}^{\prime} \tilde{\mathcal{E}}^{\prime}$.

In 27.1 replace the diagram $N_{D} P / U_{P} \stackrel{\stackrel{a}{=}}{=} V_{1} \stackrel{a}{\rightarrow} V_{2} \stackrel{a^{\prime}}{\longrightarrow} D$ by $N_{D} P / U_{P} \stackrel{\underline{a}}{\underline{a}} V_{1} \stackrel{a^{\prime}}{\longrightarrow}$ $V_{2} \stackrel{a^{\prime \prime}}{\longrightarrow} D$.

Received by the editors November 9, 2006 and, in revised form, January 21, 2006.

2000 Mathematics Subject Classification. Primary 20G99.

Supported in part by the National Science Foundation.

(C)2006 American Mathematical Society
Reverts to public domain 28 years from publication 314 
In the commutative diagram on p. 372 replace $\hat{Z}_{1} \stackrel{a}{\rightarrow} \hat{Z}_{2}, Z_{1} \stackrel{b}{\rightarrow} Z_{2}, V_{1} \stackrel{c}{\rightarrow} V_{2} \stackrel{a^{\prime}}{\longrightarrow}$ $D$ by $\hat{Z}_{1} \stackrel{a^{\prime}}{\longrightarrow} \hat{Z}_{2}, Z_{1} \stackrel{b^{\prime}}{\longrightarrow} Z_{2}, V_{1} \stackrel{c^{\prime}}{\longrightarrow} V_{2} \stackrel{a^{\prime \prime}}{\longrightarrow} D$ respectively.

Errata to Part VI.

In 28.5 replace $t_{0}: \operatorname{Ad}\left(d^{-1} \dot{w}^{-1}\right)\left(t_{0}\right) t t_{0}^{-1}$ by $t_{0}: t \mapsto \operatorname{Ad}\left(d^{-1} \dot{w}^{-1}\right)\left(t_{0}\right) t t_{0}^{-1}$.

In 28.19 (a) replace $\hat{Z}_{\emptyset, \epsilon_{D}(J), D^{-1}}^{\mathcal{L}^{\prime}}$ by $\hat{Z}_{\epsilon_{D}(J), D^{-1}}^{\mathcal{L}^{\prime}}$ (twice).

In 31.4 , line 4 replace $\mathfrak{a}_{D}^{i}$ by $\mathfrak{a}_{D}$.

Errata to Part VII.

In 32.5 replace $>b_{2}$ by $b_{2}$.

In 32.15 replace $C_{u}$ by $C^{u}$.

In $32.18\left(\right.$ a) replace $T_{\epsilon^{\prime}\left(a_{r+r^{\prime}}^{-1}\right.}$ by $T_{\epsilon^{\prime}\left(a_{r+r^{\prime}}^{-1}\right)}$.

In 32.23 replace the first $=$ by $)=$.

After the statement of Corollary 32.23 insert: Take $D^{\prime}=D^{-1}$ so that $\Delta=G^{0}$.

In the third line of Corollary 32.24 replace $\lambda^{\prime}$ by $\lambda^{\prime \prime}$.

In the fourth line of Corollary 32.24 replace $\lambda^{\prime \prime}$ by $\mathcal{L}^{\prime \prime}$.

In 32.26 , line 13 , replace $t T_{x}$ by $\tilde{T}_{x}$.

In 35.6, line 8, after $h_{i-1}^{-1} h_{i} \in U^{*} n_{i} U^{*}$, add: $(i \in[1, r])$.

\section{Contents}

36. The algebra $\underline{\mathfrak{K}}^{J}$.

37. A Mackey type formula.

38. Duality.

39. Quasi-rationality.

\section{The ALGEBRA $\underline{\mathfrak{K}}^{J}$}

36.1. We fix a connected component $D$ of $G$. We write $\epsilon$ instead of $\epsilon_{D}: \mathbf{W} \rightarrow \mathbf{W}$ (see 26.2). Occassionally we shall consider one (or two) other component(s), say $D^{\prime}$ (or $D^{\prime}, D^{\prime \prime}$ ); we write $\epsilon^{\prime}$ instead of $\epsilon_{D^{\prime}}$.

For $J \subset \mathbf{I}$ we identify $Z_{J, D}$ (see 26.2) with

$$
\left\{\left(P, g U_{P}\right) ; P \in \mathcal{P}_{J}, g U_{P} \in D / U_{P}\right\}
$$

by $\left(P, P^{\prime}, g U_{P}\right) \mapsto\left(P, g U_{P}\right)$. To any $\left(P, g U_{P}\right) \in Z_{J, D}$ we associate an element $w_{P, g U_{P}} \in \mathbf{W}$ by the following requirements. Let $z=\operatorname{pos}\left(g P g^{-1}, P\right) \in{ }^{\epsilon(J)} \mathbf{W}^{J}$; then

(i) $w_{P, g U_{P}}=w_{P_{1}, g U_{P_{1}}}$ where $P_{1}=\left(g^{-1} P g \cap P\right) U_{P} \in \mathcal{P}_{J \cap \epsilon^{-1}(\operatorname{Ad}(z) J)}$,

(ii) $w_{P, g U_{P}}=z$ if $\epsilon^{-1}(\operatorname{Ad}(z) J)=J$.

Note that (i), (ii) define uniquely $w_{P, g U_{P}}$ by induction on $|J|$ : if $|J|=0$, then $w_{P, g U_{P}}$ is given by (ii); if $|J| \geq 1$ and $\epsilon^{-1}(\operatorname{Ad}(z) J)=J$, then $w_{P, g U_{P}}$ is again given by (ii); if $|J| \geq 1$ and $\epsilon^{-1}(\operatorname{Ad}(z) J) \neq J$, then $\left|J \cap \epsilon^{-1}(\operatorname{Ad}(z) J)\right|<|J|$ and $w_{P, g U_{P}}$ is determined by (i) since $w_{P_{1}, g U_{P_{1}}}$ is known from the induction hypothesis.

From definitions we see that the map $Z_{J, D} \rightarrow \mathbf{W},\left(P, g U_{P}\right) \mapsto w_{P, g U_{P}}$ is the composition of $\beta^{\prime}: Z_{J, D} \rightarrow \mathcal{T}(J, \epsilon)$ in 26.2 (see also [L10, 3.11]) with the bijection $\mathcal{T}(J, \epsilon) \rightarrow{ }^{\epsilon(J)} \mathbf{W}$ given by [L10, 2.4, 2.5] and with the inclusion ${ }^{\epsilon(J)} \mathbf{W} \rightarrow \mathbf{W}$. In particular, we have $w_{P, g U_{P}} \in{ }^{\epsilon(J)} \mathbf{W}$ and $\operatorname{pos}\left(g P g^{-1}, P\right)=\min \left(\mathbf{W}_{\epsilon(J)} w_{P, g U_{P}} \mathbf{W}_{J}\right)$ for any $\left(P, g U_{P}\right) \in Z_{J, D}$. (For any $\mathbf{W}_{\epsilon(J)}, \mathbf{W}_{J}$ double coset $\Omega$ in $\mathbf{W}$ we denote by $\min (\Omega)$ the unique element of minimal length in $\Omega$.) 
For any $J \subset \mathbf{I}, w \in{ }^{\epsilon(J)} \mathbf{W}$, we set

$$
{ }^{w} Z_{J, D}=\left\{\left(P, g U_{P}\right) \in Z_{J, D} ; w_{P, g U_{P}}=w\right\} .
$$

Then $Z_{J, D}=\bigcup_{w \in \epsilon(J)} \mathbf{W}{ }^{w} Z_{J, D}$ is a partition; it is the same as the partition $Z_{J, D}=$ $\bigcup_{\mathbf{t} \in \mathcal{T}(J, \epsilon)} \mathbf{t}_{J, D}$ in 26.2 (see also [L10, 3.11]). In particular, each ${ }^{w} Z_{J, D}$ is a locally closed, smooth subvariety of $Z_{J, D}$ stable under the $G^{0}$-action $h:\left(P, g U_{P}\right) \mapsto$ $\left(h P h^{-1}, h g h^{-1} U_{h P h^{-1}}\right)$.

From definitions, for $J \subset \mathbf{I}, w \in{ }^{\epsilon(J)} \mathbf{W}$ we have a map

$$
\vartheta_{J, w}:{ }^{w} Z_{J, D} \rightarrow{ }^{w} Z_{J_{1}, D},\left(P, g U_{P}\right) \mapsto\left(P_{1}, g U_{P_{1}}\right)
$$

with $J_{1}=J \cap \epsilon^{-1}(\operatorname{Ad}(w) J),. w .=\min \left(\mathbf{W}_{\epsilon(J)} w \mathbf{W}_{J}\right), P_{1}=\left(g^{-1} P g \cap P\right) U_{P}$. This map may be identified with a map $\vartheta$ as in [L10, 3.11]; in particular, it is an affine space bundle (see [L10, 3.12(b)]).

36.2. If $J \subset \mathbf{I}$ and $w \in{ }^{\epsilon(J)} \mathbf{W}$ satisfies $\epsilon^{-1}(\operatorname{Ad}(w) J)=J$ (hence $w \in{ }^{\epsilon(J)} \mathbf{W}^{J}$ ), we have ${ }^{w} Z_{J, D}=\left\{\left(P, g U_{P}\right) \in Z_{J, D}, \operatorname{pos}\left(g P g^{-1}, P\right)=w\right\}$. In this case we pick $P \in \mathcal{P}_{J}, P^{\prime} \in \mathcal{P}_{\epsilon(J)}$ such that $\operatorname{pos}\left(P^{\prime}, P\right)=w$ and a common Levi $L$ of $P^{\prime}, P$. Let $\mathbf{d}=\left\{g \in D ; g L g^{-1}=L, g P g^{-1}=P^{\prime}\right\}$, a connected component of the reductive group $N_{G} L$ with identity component $L$. We have a diagram

$$
\mathbf{d} \stackrel{p r_{2}}{\longleftarrow} G^{0} /\left(U_{P} \cap U_{P^{\prime}}\right) \times \mathbf{d} \stackrel{j}{\rightarrow}{ }^{w} Z_{J, D}
$$

where $j\left(h\left(U_{P} \cap U_{P^{\prime}}\right), g\right)=\left(h P h^{-1}, h g h^{-1} U_{h P h}\right)$ is a principal $L$-bundle for an $L$ action on $G^{0} /\left(U_{P} \cap U_{P^{\prime}}\right) \times \mathbf{d}$ compatible under $p r_{2}$ with the $L$-action on $\mathbf{d}$ (by conjugation in $N_{G} L$ ). If $X$ is a character sheaf on $\mathbf{d}$, then $\operatorname{pr}_{2}^{\star} X$ is therefore an $L$-equivariant simple perverse sheaf on $G^{0} /\left(U_{P} \cap U_{P^{\prime}}\right) \times \mathbf{d}$ which must be of the form $j^{\star} X^{\prime}$ for a well-defined simple perverse sheaf $X^{\prime}$ on ${ }^{w} Z_{J, D}$.

The collection of simple perverse sheaves on ${ }^{w} Z_{J, D}$ of the form $X^{\prime}$ with $X$ as above is denoted by ${ }^{w} \hat{Z}_{J, D}$. This collection is independent of the choice of $P, P^{\prime}, L$. Note that $X \mapsto X^{\prime}$ defines a bijection between the set of isomorphism classes of character sheaves on $\mathbf{d}$ and the set of isomorphism classes of objects in ${ }^{w} \hat{Z}_{J, D}$.

36.3. More generally, for $J \subset \mathbf{I}$ and $w \in{ }^{\epsilon(J)} \mathbf{W}$, we define by induction on $|J|$ a collection of simple $G^{0}$-equivariant perverse sheaves ${ }^{w} \hat{Z}_{J, D}$ on ${ }^{w} Z_{J, D}$.

If $|J|=0$, then ${ }^{w} \hat{Z}_{J, D}$ is defined as in 36.2. If $|J| \geq 1$ and $\epsilon^{-1}(\operatorname{Ad}(w) J)=J$, then ${ }^{w} \hat{Z}_{J, D}$ is again defined as in 36.2. If $|J| \geq 1$ and $\epsilon^{-1}(\operatorname{Ad}(w) J) \neq J$, then $\epsilon^{-1}(\operatorname{Ad}(w) J) \neq$.$J where w .=\min \left(\mathbf{W}_{\epsilon(J)} w \mathbf{W}_{J}\right)$. Thus, if $J_{1}=J \cap \epsilon^{-1}(\operatorname{Ad}(w) J$.$) ,$ then $\left|J_{1}\right|<|J|$ and the class of perverse sheaves ${ }^{w} \hat{Z}_{J_{1}, D}$ on ${ }^{w} Z_{J_{1}, D}$ is defined from the induction hypothesis. By definition, ${ }^{w} \hat{Z}_{J, D}$ consists of the simple perverse sheaves on ${ }^{w} Z_{J, D}$ of the form $\vartheta_{J, w}^{\star}(X)$ for some $X \in{ }^{w} Z_{J_{1}, D}$ (with $\vartheta_{J, w}$ as in 36.1). This completes the inductive definition of ${ }^{w} \hat{Z}_{J, D}$. The objects of ${ }^{w} \hat{Z}_{J, D}$ are said to be character sheaves on ${ }^{w} Z_{J, D}$. Let $\mathcal{I}_{J, w, D}$ be a set of representatives for the isomorphism classes of character sheaves on ${ }^{w} Z_{J, D}$.

For $J \subset \mathbf{I}$ and $w \in{ }^{\epsilon(J)} \mathbf{W}$, let $\mathcal{D}^{c s}\left({ }^{w} Z_{J, D}\right)$ be the subcategory of $\mathcal{D}\left({ }^{w} Z_{J, D}\right)$ whose objects are those $K \in \mathcal{D}\left({ }^{w} Z_{J, D}\right)$ such that for any $j$, any simple subquotient of ${ }^{p} H^{j} K$ is in ${ }^{w} \hat{Z}_{J, D}$. Let $i_{J, w}:{ }^{w} Z_{J, D} \rightarrow Z_{J, D}$ be the inclusion.

(a) If $K \in \mathcal{D}^{c s}\left(Z_{J, D}\right)$, then $i_{J, w}^{*}(K) \in \mathcal{D}^{c s}\left({ }^{w} Z_{J, D}\right)$.

It is enough to prove (a) for $K \in \hat{Z}_{J, D}$. In this case (a) follows from [L10, 4.12]. 
For $J \subset \mathbf{I}, w \in{ }^{\epsilon(J)} \mathbf{W}$ and $K \in{ }^{w} \hat{Z}_{J, D}$, let $K^{\sharp}$ be the unique simple perverse sheaf on $Z_{J, D}$ such that $i_{J, w}^{*}\left(K^{\sharp}\right)=K$ and $\operatorname{supp}\left(K^{\sharp}\right)$ is the closure in $Z_{J, D}$ of $\operatorname{supp}(K)$.

(b) We have $K^{\sharp} \in \hat{Z}_{J, D}$.

This is proved in [L10, 4.17] based on the following statement:

(c) Let $Y$ be a locally closed subvariety of an algebraic variety $Y^{\prime}$ and let $i: Y \rightarrow$ $Y^{\prime}$ be the inclusion. Let $C \in \mathcal{D}(Y)$ and let $A$ be a simple perverse sheaf on $Y$ such that $A \dashv C$. Let $A^{\sharp}$ be the unique simple perverse sheaf on $Y$ such that $i^{*}\left(A^{\sharp}\right)=A$ and $\operatorname{supp}\left(A^{\sharp}\right)$ is the closure in $Y^{\prime}$ of $\operatorname{supp}(A)$. Then $A^{\sharp} \dashv i_{!} C$.

(In loc.cit. this is applied with $Y={ }^{w} Z_{J, D}, Y^{\prime}=Z_{J, D}, A=K$ and an $C \in$ $\mathcal{D}^{c s}\left(Z_{J, D}\right)$.) Since the proof of (c) is omitted in loc.cit. we give a proof here. Let $Y_{1}$ be the closure of $Y$ in $Y^{\prime}$. Let $Y \stackrel{i_{1}}{\longrightarrow} Y_{1} \stackrel{i_{2}}{\longrightarrow} Y^{\prime}$ be the inclusions. Clearly, if (c) holds for $i_{1}$ and $i_{2}$ instead of $i$, then it holds for $i$. Thus we may assume that $i=i_{1}$ or $i=i_{2}$, that is, that $Y$ is open or closed in $Y^{\prime}$. Assume first that $Y$ is

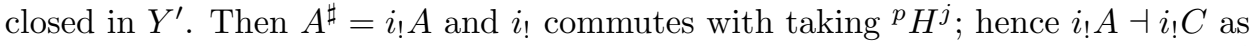
desired. Next, assume that $Y$ is open in $Y^{\prime}$. Then $i^{*}$ commutes with taking ${ }^{p} H^{j}$ and $i^{*} i_{l} C=C$. Hence for any $j$ we have ${ }^{p} H^{j}(C)=i^{*}\left({ }^{p} H^{j}\left(i_{!} C\right)\right)$ and for some $j$ we have $A \dashv i^{*}\left({ }^{p} H^{j}\left(i_{!} C\right)\right)$. Let $0=F_{0} \subset F_{1} \subset \ldots \subset F_{m}={ }^{p} H^{j}\left(i_{!} C\right)$ be a sequence of perverse subsheaves of ${ }^{p} H^{j}\left(i_{!} C\right)$ such that $F_{k} / F_{k-1}$ is simple for $k \in[1, m]$. Then $0=i^{*} F_{0} \subset i^{*} F_{1} \subset \ldots \subset i^{*} F_{m}=i^{*}\left({ }^{p} H^{j}\left(i_{!} C\right)\right)$ is a sequence of perverse subsheaves of $i^{*}\left({ }^{p} H^{j}\left(i_{!} C\right)\right)$ such that $i^{*} F_{k} / i^{*} F_{k-1}=i^{*}\left(F_{k} / F_{k-1}\right)$ is simple or 0 for $k \in[1, m]$. Hence $A \cong i^{*} F_{k} / i^{*} F_{k-1}=i^{*}\left(F_{k} / F_{k-1}\right)$ for some $k \in[1, m]$. Then $A^{\prime}=F_{k} / F_{k-1}$ is a simple perverse sheaf on $Y^{\prime}$ such that $A^{\prime} \dashv i_{1} C, i^{*} A^{\prime} \cong A$. We must have $A^{\prime} \cong A^{\sharp}$ and $(\mathrm{c})$ is proved.

(d) Let $K^{\prime} \in \hat{Z}_{J, D}$. There exists a unique $w \in{ }^{\epsilon(J)} \mathbf{W}$ and a unique $K \in{ }^{w} \hat{Z}_{J, D}$ (up to isomorphism) such that $K^{\prime} \cong K^{\sharp}$.

This is proved in [L10,4.13]; it is an immediate consequence of (a).

Let $\mathcal{I}_{J, D}=\bigsqcup_{w \in \epsilon(J)} \mathbf{W}\left\{K^{\sharp} ; K \in \mathcal{I}_{J, w, D}\right\}$.

From (b), (d) we see that

(e) $\mathcal{I}_{J, D}$ is a set of representatives for the isomorphism classes of character sheaves on $Z_{J, D}$.

From (a), (b) we deduce:

(f) If $w \in{ }^{\epsilon(J)} \mathbf{W}$ and $K \in \mathcal{D}^{c s}\left({ }^{w} Z_{J, D}\right)$, then $\left(i_{J, w}\right) ! K \in \mathcal{D}^{c s}\left(Z_{J, D}\right)$.

36.4. For $J \subset J^{\prime} \subset \mathbf{I}$ and $P \in \mathcal{P}_{J}$ let $Q_{J^{\prime}, P}$ be the unique parabolic in $\mathcal{P}_{J^{\prime}}$ that contains $P$. We have a diagram

$$
Z_{J, D} \stackrel{\mathfrak{c}}{\leftarrow} Z_{J, J^{\prime}, D} \stackrel{\mathfrak{o}}{\rightarrow} Z_{J^{\prime}, D}
$$

where

$$
\begin{gathered}
Z_{J, J^{\prime}, D}=\left\{\left(P, g U_{Q}\right) ; P \in \mathcal{P}_{J}, Q=Q_{J^{\prime}, P}, g U_{Q} \in D / U_{Q}\right\} \\
\mathfrak{c}\left(P, g U_{Q}\right)=\left(P, g U_{P}\right), \mathfrak{d}\left(P, g U_{Q}\right)=\left(Q, g U_{Q}\right) .
\end{gathered}
$$

Define functors

$$
\mathfrak{f}_{J, J^{\prime}}: \mathcal{D}\left(Z_{J, D}\right) \rightarrow \mathcal{D}\left(Z_{J^{\prime}, D}\right), \mathfrak{e}_{J, J^{\prime}}: \mathcal{D}\left(Z_{J^{\prime}, D}\right) \rightarrow \mathcal{D}\left(Z_{J, D}\right)
$$

by $\mathfrak{f}_{J, J^{\prime}} A=\mathfrak{d}_{!} \mathfrak{c}^{*} A, \mathfrak{e}_{J, J^{\prime}} A^{\prime}=\mathfrak{c} ! \mathfrak{d}^{*} A^{\prime}$. Now $\mathfrak{d}$ is proper and $\mathfrak{c}$ is an affine space bundle with fibres of dimension $a=\operatorname{dim} \mathcal{P}_{J}-\operatorname{dim} \mathcal{P}_{J^{\prime}}$. Hence $\mathfrak{f}_{J, J^{\prime}}$ commutes with Verdier 
duality up to a shift and a twist:

(a)

$$
\mathfrak{D f}_{J, J^{\prime}}=\mathfrak{f}_{J, J^{\prime}}[[a]] \mathfrak{D}: \mathcal{D}\left(Z_{J, D}\right) \rightarrow \mathcal{D}\left(Z_{J^{\prime}, D}\right) .
$$

For $J \subset J^{\prime} \subset J^{\prime \prime} \subset \mathbf{I}$ we have (see [L10, 6.2]):

$$
\mathfrak{f}_{J, J^{\prime \prime}}=\mathfrak{f}_{J^{\prime}, J^{\prime \prime}} \mathfrak{f}_{J, J^{\prime}}, \mathfrak{e}_{J, J^{\prime \prime}}=\mathfrak{e}_{J, J^{\prime}} \mathfrak{e}_{J^{\prime}, J^{\prime \prime}} .
$$

Clearly, $\mathfrak{f}_{J, J}=1, \mathfrak{e}_{J, J}=1$.

For $J \subset J^{\prime} \subset \mathbf{I}$ we show that the convolution bifunctor

$$
\mathcal{D}\left(Z_{J, D}\right) \times \mathcal{D}\left(Z_{\epsilon(J), D^{\prime}}\right) \rightarrow \mathcal{D}\left(Z_{J, D^{\prime} D}\right), A, B \mapsto A * B,
$$

(see 32.5) is compatible with the functors $\mathfrak{e}_{J, J^{\prime}}$ in the following sense: for $A \in$ $\mathcal{D}\left(Z_{J, D}\right), A^{\prime} \in \mathcal{D}\left(Z_{\epsilon(J), D^{\prime}}\right)$, we have

$$
\left(\mathfrak{e}_{J, J^{\prime}} A\right) *\left(\mathfrak{e}_{J, J^{\prime}} A^{\prime}\right)=\mathfrak{e}_{J, J^{\prime}}\left(A * A^{\prime}\right) .
$$

We have a commutative diagram

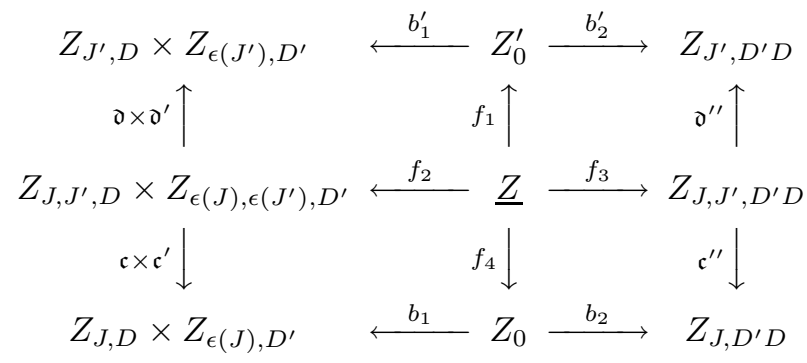

Here $\mathfrak{c}, \mathfrak{d}$ are as above,

$$
Z_{\epsilon\left(J^{\prime}\right), D^{\prime}} \stackrel{\mathfrak{c}^{\prime}}{\longleftarrow} Z_{\epsilon(J), \epsilon\left(J^{\prime}\right), D^{\prime}} \stackrel{\mathfrak{d}^{\prime}}{\longrightarrow} Z_{\epsilon\left(J^{\prime}\right), D^{\prime}}, Z_{J, D^{\prime} D} \stackrel{\mathfrak{c}^{\prime \prime}}{\longleftarrow} Z_{J, J^{\prime}, D^{\prime} D} \stackrel{\stackrel{\mathfrak{d}^{\prime \prime}}{\longrightarrow}}{\longrightarrow} Z_{J^{\prime}, D^{\prime} D}
$$

are the analogous maps when $\left(J, J^{\prime}, D\right)$ is replaced by $\left(\epsilon(J), \epsilon\left(J^{\prime}\right), D^{\prime}\right)$ or by $\left(J, J^{\prime}, D^{\prime} D\right), b_{1}, b_{2}$ are as in $32.5, b_{1}^{\prime}, b_{2}^{\prime}$ are the analogous maps with $J$ replaced by $J^{\prime}$,

$$
\begin{array}{r}
\underline{Z}=\left\{\left(X, P, g U_{P}, g^{\prime} U_{P^{\prime}}\right) ; X \in \mathcal{P}_{J}, P=Q_{J^{\prime}, X}, g U_{P} \in D / U_{P},\right. \\
\left.P^{\prime}=g P g^{-1}, g^{\prime} U_{P^{\prime}} \in D^{\prime} / U_{P^{\prime}}\right\}, \\
f_{1}\left(X, g U_{P}, g^{\prime} U_{P^{\prime}}\right)=\left(P, g P g^{-1}, g^{\prime} g P g^{-1} g^{\prime-1}, g U_{P}, g^{\prime} U_{g P g^{-1}}\right), \\
f_{2}\left(X, g U_{P}, g^{\prime} U_{P^{\prime}}\right)=\left(\left(X, g U_{P}\right),\left(g X g^{-1}, g^{\prime} U_{g P g^{-1}}\right)\right), \\
f_{3}\left(X, g U_{P}, g^{\prime} U_{P^{\prime}}\right)=\left(X, g^{\prime} g U_{P}\right), \quad \\
f_{4}\left(X, g U_{P}, g^{\prime} U_{P^{\prime}}\right)=\left(X, g X g^{-1}, g^{\prime} g X g^{-1} g^{\prime-1}, g U_{X}, g^{\prime} U_{g X g^{-1}}\right) .
\end{array}
$$

It is enough to show that

$$
b_{2 !} b_{1}^{*}\left(\mathfrak{c} \times \mathfrak{c}^{\prime}\right) !\left(\mathfrak{d} \times \mathfrak{d}^{\prime}\right)^{*}\left(A \otimes A^{\prime}\right)=\mathfrak{c}_{!}^{\prime \prime} \mathfrak{d}^{\prime \prime *} b_{2 !}^{\prime} b_{1}^{\prime *}\left(A \otimes A^{\prime}\right) .
$$

Since the upper right and lower left squares are cartesian, we have

$$
b_{1}^{*}\left(\mathfrak{c} \times \mathfrak{c}^{\prime}\right) !=f_{4 !} f_{2}^{*}, \mathfrak{d}^{\prime \prime *} b_{2 !}^{\prime}=f_{3 !} f_{1}^{*} .
$$

Hence it is enough to show that

$$
b_{2 !} f_{4 !} f_{2}^{*}\left(\mathfrak{d} \times \mathfrak{d}^{\prime}\right)^{*}\left(A \otimes A^{\prime}\right)=\mathfrak{c}_{!}^{\prime \prime} f_{3 !} f_{1}^{*} b_{1}^{\prime *}\left(A \otimes A^{\prime}\right) .
$$

This follows from the commutativity of the diagram above: we have $b_{2 !} f_{4 !}=\mathfrak{c}_{!}^{\prime \prime} f_{3}$ ! and $f_{2}^{*}\left(\mathfrak{d} \times \mathfrak{d}^{\prime}\right)^{*}=f_{1}^{*} b_{1}^{\prime *}$. This proves $(\mathrm{c})$. 
If $A \in \mathcal{D}\left(Z_{J, D}\right), B \in \mathcal{D}\left(Z_{\epsilon(J), D^{\prime}}\right), C \in \mathcal{D}\left(Z_{\epsilon^{\prime} \epsilon(J), D^{\prime \prime}}\right)$, then, from definitions, we have the associativity property

$$
(A * B) * C=A *(B * C) .
$$

Consider the isomorphism $\partial: Z_{J, D} \stackrel{\sim}{\longrightarrow} Z_{\epsilon(J), D^{-1}}$ as in 28.19. Note that the composition $Z_{J, D} \stackrel{\partial}{\rightarrow} Z_{\epsilon(J), D^{-1}} \stackrel{\partial}{\rightarrow} Z_{J, D}$ is the identity map. From definitions, we have for $A, B$ as above,

$$
\partial_{!}(A * B)=\left(\partial_{!} B\right) *\left(\partial_{!} A\right) .
$$

For $J \subset \mathbf{I}$ we define a functor $\tau: \mathcal{D}\left(Z_{J, G^{0}}\right) \rightarrow \mathcal{D}$ (point) by $\tau(C)=p_{!}^{\prime} i^{*}(C)$ where $i: \mathcal{P}_{J} \rightarrow Z_{J, G^{0}}$ is the imbedding $P \mapsto\left(P, U_{P}\right)$ and $p^{\prime}: \mathcal{P}_{J} \rightarrow$ point is the obvious map. We define a bifunctor $(:): \mathcal{D}\left(Z_{J, D}\right) \times \mathcal{D}\left(Z_{J, D}\right) \rightarrow \mathcal{D}$ (point) by $(A: B)=$ $p ! i^{\prime *}(A \otimes B)$ where $i^{\prime}: Z_{J, D} \rightarrow Z_{J, D} \times Z_{J, D}$ is the diagonal and $p: Z_{J, D} \rightarrow$ point is the obvious map. As in 32.23(b) we have

$$
(A: B)=\tau\left(A *\left(\partial_{!} B\right)\right) .
$$

From definitions we have

$$
(A: B)=\left(\partial_{!} A: \partial_{!} B\right)
$$

If $A \in \mathcal{D}\left(Z_{J, D}\right), B \in \mathcal{D}\left(Z_{\epsilon(J), D^{\prime}}\right), C \in \mathcal{D}\left(Z_{\epsilon^{\prime} \epsilon(J), D^{-1} D^{\prime-1}}\right)$, then from (d) we have $\tau((A * B) * C)=\tau(A *(B * C))$. Using (f) we rewrite this as $\left(A * B: \partial_{!} C\right)=$ $\left(A: \partial_{\mathrm{l}}(B * C)\right)$ or, using (g), as

$$
\left(A * B: \partial_{!} C\right)=\left(\partial_{!} A: B * C\right) .
$$

For $J \subset J^{\prime}, \mathfrak{c}, \mathfrak{d}$ as above and $A \in \mathcal{D}\left(Z_{J, D}\right), B \in \mathcal{D}\left(Z_{J^{\prime}, D}\right)$ we show that

$$
\left(B: f_{J, J^{\prime}} A\right)=\left(e_{J, J^{\prime}} B: A\right) \in \mathcal{D}(\text { point }) .
$$

Let $p_{1}, p_{2}, p_{3}$ be the obvious maps from $Z_{J^{\prime}, D}, Z_{J, D}, Z_{J, J^{\prime}, D}$ to the point. We must show that $p_{1 !}\left(B \otimes \mathfrak{d}_{!} \mathfrak{c}^{*} A\right)=p_{2 !}\left(\mathfrak{c} ! \mathbb{d}^{*} B \otimes A\right)$. Since $p_{1 !} \mathfrak{d}_{!}=p_{3 !}=p_{2 !} \mathfrak{c}$, both sides are equal to $p_{3 !}\left(\mathfrak{d}^{*} B \otimes \mathfrak{c}^{*} A\right)$. This proves (i).

36.5. For $J \subset J^{\prime} \subset \mathbf{I}$ and $w \in{ }^{\epsilon(J)} \mathbf{W}, w^{\prime} \in{ }^{\epsilon\left(J^{\prime}\right)} \mathbf{W}$ we have a diagram

$$
{ }^{w} Z_{J, D} \stackrel{\mathfrak{c}_{w, w^{\prime}}}{\longleftarrow} w^{w} w^{\prime} Z_{J, J^{\prime}, D} \stackrel{\mathfrak{o}_{w, w^{\prime}}}{\longrightarrow} w^{\prime} Z_{J^{\prime}, D}
$$

where

$$
{ }^{w, w^{\prime}} Z_{J, J^{\prime}, D}=\left\{\left(P, g U_{Q}\right) \in Z_{J, J^{\prime}, D} ;\left(P, g U_{P}\right) \in{ }^{w} Z_{J, D},\left(Q, g U_{Q}\right) \in{ }^{w^{\prime}} Z_{J^{\prime}, D}\right\}
$$

and $\mathfrak{c}_{w, w^{\prime}}, \mathfrak{d}_{w, w^{\prime}}$ are the restrictions of $\mathfrak{c}, \mathfrak{d}$ in 36.4. Consider the functor

$$
\mathfrak{f}_{J, w, J^{\prime}, w^{\prime}}: \mathcal{D}\left({ }^{w} Z_{J, D}\right) \rightarrow \mathcal{D}\left({ }^{w^{\prime}} Z_{J^{\prime}, D}\right), A \mapsto \mathfrak{d}_{w, w^{\prime} !} \mathfrak{c}_{w, w^{\prime}}^{*} A .
$$

From definitions we see that, for $A \in \mathcal{D}\left({ }^{w} Z_{J, D}\right)$, we have

$$
\mathfrak{f}_{J, w, J^{\prime}, w^{\prime}}(A)=i_{J^{\prime}, w^{\prime}}^{*} \mathfrak{f}_{J, J^{\prime}}\left(i_{J, w !} A\right) .
$$


36.6. Let $J \subset \mathbf{I}$ and let $w \in{ }^{\epsilon(J)} \mathbf{W}$. We have a sequence of affine space bundles

$$
{ }^{w} Z_{J, D} \stackrel{\vartheta_{J, w}}{\longrightarrow}{ }^{w} Z_{J_{1}, D} \stackrel{\vartheta_{J_{1}, w}}{\longrightarrow}{ }^{w} Z_{J_{2}, D} \stackrel{\vartheta_{J_{2}, w}}{\longrightarrow} \ldots
$$

where $\vartheta_{J, w}$ is as in $36.1, \vartheta_{J_{1}, w}$ is the analogous map with $J$ replaced by $J_{1}$ (we set $\left.\left(J_{1}\right)_{1}=J_{2}\right), \vartheta_{J_{2}, w}$ is the analogous map with $J$ replaced by $J_{2}$ (we set $\left(J_{2}\right)_{1}=J_{3}$ ), etc. We have $J \supset J_{1} \supset J_{2} \supset \ldots$ Let $J_{\infty}=J_{r}$ for large $r$. Since $\vartheta_{J_{r}, w}=1$ for large $r$, the composition of the maps (a) is a well-defined map $\underline{\vartheta}:{ }^{w} Z_{J, D} \rightarrow{ }^{w} Z_{J_{\infty}, D}$. For any $\left(P, g U_{P}\right) \in{ }^{w} Z_{J, D}$ we have

$$
\vartheta_{J, w}\left(P, g U_{P}\right)=\left(P_{1}, g U_{P}\right), \vartheta_{J_{1}, w}\left(P_{1}, g U_{P_{1}}\right)=\left(P_{2}, g U_{P_{2}}\right), \ldots
$$

where $P \supset P_{1} \supset P_{2} \supset \ldots$ Let $P_{\infty}=P_{r}$ for large $r$. We show:

(b) The map $\alpha:{ }^{w, w} Z_{J_{\infty}, J, D} \rightarrow{ }^{w} Z_{J, D},\left(R, g U_{P}\right) \mapsto\left(P, g U_{P}\right)$, is an isomorphism.

We show only that $\alpha$ is a bijection. Let $\left(P, g U_{P}\right) \in{ }^{w} Z_{J, D}$. By [L10, 4.14(b)] there exists $(B, h) \in \mathcal{B} \times g U_{P}$ such that $B \subset P$ and $\operatorname{pos}\left(h B h^{-1}, B\right)=w$. We have also $\operatorname{pos}\left(g B g^{-1}, B\right)=w$. Let $R=Q_{J_{\infty}, B}$. By [L10, 4.14(a)] we have $\left(R, g U_{R}\right) \in$ ${ }^{w} Z_{J_{\infty}, D}$. We have $\left(R, g U_{P}\right) \in{ }^{w, w} Z_{J_{\infty}, J, D}$ and $\alpha\left(R, g U_{P}\right)=\left(P, g U_{P}\right)$. Thus $\alpha$ is surjective.

We show that $\alpha$ is injective. Let $\left(R, g U_{P}\right) \in{ }^{w, w} Z_{J_{\infty}, J, D}$. Define $P_{1}, P_{2}, \ldots, P_{\infty}$ in terms of $\left(P, g U_{P}\right)$ as above. It is enough to show that $R=P_{\infty}$. Let $P^{\prime}=$ $g P g^{-1}, R^{\prime}=g R g^{-1}$. Since $\left(R, g U_{R}\right) \in{ }^{w} Z_{J_{\infty}, D}$ and $\epsilon^{-1}\left(\operatorname{Ad}(w) J_{\infty}\right)=J_{\infty}$, we see that $R, R^{\prime}$ have a common Levi and $\operatorname{pos}\left(R^{\prime}, R\right)=w$. Hence if $B^{\prime}$ is any Borel of $R^{\prime}$, there exists a Borel $B$ of $R$ such that $\operatorname{pos}\left(B^{\prime}, B\right)=w$. If $w$. $=\operatorname{pos}\left(P^{\prime}, P\right)$, we have $w=w \cdot x$ where $x \in \mathbf{W}_{J}$ (see [L10, 2.1(b)]) and $l(w)+.l(x)=l(w)$. Hence we can find a Borel $B_{1}$ of $G^{0}$ such that $\operatorname{pos}\left(B^{\prime}, B_{1}\right)=w$., $\operatorname{pos}\left(B_{1}, B\right)=x$. Since $B \subset R \subset P$, we have $B_{1} \subset P$. Since $\operatorname{pos}\left(B^{\prime}, B_{1}\right)=\operatorname{pos}\left(P^{\prime}, P\right)=w$., we have $B^{\prime} \subset\left(P^{\prime} \cap P\right) U_{P^{\prime}}$ (see [L10, 2.7]). Since this holds for any Borel $B^{\prime}$ of $R^{\prime}$ and $R^{\prime}$ is the union of its Borels, it follows that $R^{\prime} \subset\left(P^{\prime} \cap P\right) U_{P^{\prime}}$. Hence $R=g^{-1} R^{\prime} g \subset\left(g^{-1} P g \cap P\right) U_{P}=P_{1}$. Hence $\left(R, g U_{P_{1}}\right) \in{ }^{w, w} Z_{J_{\infty}, J_{1}, D}$ is well defined. Repeating the previous argument for $\left(R, g U_{P_{1}}\right)$ instead of $\left(R, g U_{P}\right)$ we see that $R \subset P_{2}$. Continuing in this way we obtain $R \subset P_{r}$ for any $r \geq 0$. In particular, $R \subset P_{\infty}$. Since $R, P_{\infty}$ are parabolics of the same type, we see that $R=P_{\infty}$. This proves (b).

Next we show that

(c) if $y \in{ }^{\epsilon(J)} \mathbf{W}, y \neq w$, then ${ }^{w, y} Z_{J_{\infty}, J, D}=\emptyset$.

Assume that $\left(R, g U_{P}\right) \in{ }^{w, y} Z_{J_{\infty}, J, D}$. We have $\left(R, g U_{R}\right) \in{ }^{w} Z_{J_{\infty}, D}$ and by L10, 4.14(b)] there exists $(B, h) \in \mathcal{B} \times g U_{R}$ such that $B \subset R$ and $\operatorname{pos}\left(h B h^{-1}, B\right)=w$. We have also $\operatorname{pos}\left(g B g^{-1}, B\right)=w$. Since $B \subset P$ and $w \in{ }^{\epsilon(J)} \mathbf{W}$, we see, using [10, 4.14(a)], that $\left(P, g U_{P}\right) \in{ }^{w} Z_{J, D}$. Since $\left(P, g U_{P}\right) \in{ }^{y} Z_{J, D}$ and ${ }^{y} Z_{J, D},{ }^{w} Z_{J, D}$ are disjoint for $y \neq w$, we have a contradiction. This proves (c).

Let $A \in{ }^{w} \hat{Z}_{J, D}$. From definitions we have $A=\underline{\vartheta}^{\star}\left(A^{\prime}\right)$ with $A^{\prime} \in{ }^{w} \hat{Z}_{J_{\infty}, D}$. We show:

(d) $\mathfrak{f}_{J_{\infty}, w, J, w}\left(A^{\prime}\right) \cong A[-\delta]$ for some integer $\delta$.

(e) $\mathfrak{f}_{J_{\infty}, w, J, y}\left(A^{\prime}\right)=0$ for any $y \in{ }^{\epsilon(J)} \mathbf{W}, y \neq w$.

Now (e) follows immediately from (c). To prove (d) we recall that $\mathfrak{f}_{J_{\infty}, w, J, w}\left(A^{\prime}\right)=$ $\alpha_{!} \tilde{a}^{*}\left(A^{\prime}\right)$ where $\tilde{a}:{ }^{w, w} Z_{J_{\infty}, J, D} \rightarrow{ }^{w} Z_{J_{\infty}, D}$ is given by $\left(R, g U_{P}\right) \mapsto\left(R, g U_{R}\right)$. Define 
$e:{ }^{w} Z_{J, D} \rightarrow{ }^{w, w} Z_{J_{\infty}, J, D}$ by $e\left(P, g U_{P}\right)=\left(P_{\infty}, g U_{P}\right)$ with $P_{\infty}$ as above. Clearly, $\tilde{a} e=\underline{\vartheta}, \alpha e=1$. Since $\alpha$ is an isomorphism, we see that

$$
\alpha_{!} \tilde{a}^{*}\left(A^{\prime}\right)=e^{*} \tilde{a}^{*}\left(A^{\prime}\right)=\underline{\vartheta}^{*}\left(A^{\prime}\right)=\underline{\vartheta}^{\star}\left(A^{\prime}\right)[-\delta]=A[-\delta]
$$

where $\delta$ is the dimension of any fibre of $\underline{\vartheta}$. This proves (d).

36.7. Let $L \subset J \subset \mathbf{I}$ and let $w \in{ }^{\epsilon(J)} \mathbf{W}, y \in{ }^{\epsilon(L)} \mathbf{W}$. Assume that $\epsilon^{-1}(\operatorname{Ad}(w) J)=$ $J, \epsilon^{-1}(\operatorname{Ad}(y) L)=L$. From definitions we have

$$
\begin{aligned}
{ }^{y, w} Z_{L, J, D}= & \left\{\left(R, g U_{P}\right) ; R \in \mathcal{P}_{L}, P=Q_{J, R}, g U_{P} \in D / U_{P},\right. \\
& \left.\operatorname{pos}\left(g R g^{-1}, R\right)=y, \operatorname{pos}\left(g P g^{-1}, P\right)=w\right\} .
\end{aligned}
$$

We show:

(a) ${ }^{y, w} Z_{L, J, D}=\emptyset$ unless $y \in \mathbf{W}_{\epsilon(J)} w=w \mathbf{W}_{J}$, that is, $w=\min \left(\mathbf{W}_{\epsilon(J)} y \mathbf{W}_{J}\right)$.

Assume that $\left(R, g U_{P}\right) \in{ }^{y, w} Z_{L, J, D}$. Let $B$ be a Borel of $g R g^{-1}$. Then $B \subset g P g^{-1}$. In our case $g P g^{-1}, P$ have a common Levi; hence there exists a Borel $B^{\prime}$ of $P$ such that $\operatorname{pos}\left(B, B^{\prime}\right)=\operatorname{pos}\left(g P g^{-1}, P\right)=w$. Similarly, since $g R g^{-1}, R$ have a common Levi, there exists a Borel $B^{\prime \prime}$ of $R$ such that $\operatorname{pos}\left(B, B^{\prime \prime}\right)=\operatorname{pos}\left(g R g^{-1}, R\right)=y$. Let $z=\operatorname{pos}\left(B^{\prime}, B^{\prime \prime}\right)$. Since $B^{\prime} \subset P, B^{\prime \prime} \subset P$, we have $z \in \mathbf{W}_{J}$. Since $w \in \mathbf{W}^{J}$ we have $l(w z)=l(w)+l(z)$ hence from $\operatorname{pos}\left(B, B^{\prime}\right)=w, \operatorname{pos}\left(B^{\prime}, B^{\prime \prime}\right)=z$ we deduce $\operatorname{pos}\left(B, B^{\prime \prime}\right)=w z$. Thus $y=w z$ and (a) follows.

Now for $J \subset \mathbf{I}, w \in{ }^{\epsilon(J)} \mathbf{W}, w^{\prime} \in \epsilon^{\epsilon^{\prime} \epsilon(J)} \mathbf{W}, y \in \epsilon^{\epsilon^{\prime} \epsilon(J)} \mathbf{W}$, the diagram

$$
Z_{J, D} \times Z_{\epsilon(J), D^{\prime}} \stackrel{b_{1}}{\longleftarrow} Z_{0} \stackrel{b_{2}}{\longrightarrow} Z_{J, D^{\prime} D}
$$

(see 32.5$)$ restricts to a diagram

$$
{ }^{w} Z_{J, D} \times{ }^{w^{\prime}} Z_{\epsilon(J), D^{\prime}} \stackrel{b_{w, w^{\prime}, 1}}{\longleftarrow} w, w^{\prime}, y Z_{0} \stackrel{b_{w, w^{\prime}, 2}}{\longleftrightarrow} Z_{J, D^{\prime} D}
$$

where ${ }^{w, w^{\prime}, y} Z_{0}=b_{1}^{-1}\left({ }^{w} Z_{J, D} \times{ }^{w^{\prime}} Z_{\epsilon(J), D^{\prime}}\right) \cap b_{2}^{-1}\left({ }^{y} Z_{J, D^{\prime} D}\right)$. Define a bifunctor $\mathcal{D}\left({ }^{w} Z_{J, D}\right) \times \mathcal{D}\left(w^{w^{\prime}} Z_{\epsilon(J), D^{\prime}}\right) \rightarrow \mathcal{D}\left({ }^{y} Z_{J, D^{\prime} D}\right)$ by

$$
A, B \mapsto A *_{y} B=b_{w, w^{\prime}, 2 !} b_{w, w^{\prime}, 1}^{*}(A \otimes B) .
$$

Let $K \in \mathcal{D}\left({ }^{w} Z_{J, D}\right), K^{\prime} \in \mathcal{D}\left({ }^{w^{\prime}} Z_{\epsilon(J), D^{\prime}}\right)$. From definitions we see that

$$
i_{J, y}^{*}\left(\left(i_{J, w !} K\right) *\left(i_{\epsilon(J), w^{\prime} !} K^{\prime}\right)\right)=K *_{y} K^{\prime} .
$$

36.8. In the remainder of this section we assume that $\mathbf{k}$ is an algebraic closure of a finite field. As in 31.2 let $\mathcal{A}=\mathbf{Z}\left[v, v^{-1}\right], v$ an indeterminate.

Let $V$ be an algebraic variety with a given family of simple perverse sheaves (called "character sheaves") with the following property: any character sheaf on $V$ comes from a mixed complex on $V$ relative to a rational structure of $V$ over a finite subfield of $\mathbf{k}$. Let $\mathcal{D}^{c s}(V)$ be the subcategory of $\mathcal{D}(V)$ whose objects are complexes $K$ such that for any $j$, any composition factor of ${ }^{p} H^{j}(K)$ is a "character sheaf". Let $\mathfrak{K}(V)$ be the free $\mathcal{A}$-module with basis given by the isomorphism class of "character sheaves" on $V$. Let $K$ be an object of $\mathcal{D}^{c s}(V)$ with a given mixed structure relative to a rational structure of $V$ over a finite subfield of $\mathbf{k}$. We set

$$
\operatorname{gr}(K)=\sum_{A} \sum_{j, h \in \mathbf{Z}}(-1)^{j}\left(\text { multiplicity of } A \text { in }{ }^{p} H^{j}(K)_{h}\right) v^{h} A \in \mathfrak{K}(V),
$$

where $A$ runs over a set of representatives for the isomorphism classes of "character sheaves" on $V$ and the subscript $h$ denotes the subquotient of pure weight $h$ of a mixed perverse sheaf. 
Now let $V^{\prime}$ be another algebraic variety with a given family of simple perverse sheaves (called "character sheaves") like that of $V$. Then $\mathcal{D}^{c s}\left(V^{\prime}\right)$ is defined. Assume that we are given a functor $\Theta: \mathcal{D}(V) \rightarrow \mathcal{D}\left(V^{\prime}\right)$ which restricts to a functor $\mathcal{D}^{c s}(V) \rightarrow \mathcal{D}^{c s}\left(V^{\prime}\right)$. We also assume that $\Theta$ is a composition of functors of the form $a_{!}, a^{*}$ induced by various maps $a$ between algebraic varieties. In particular, $\Theta$ preserves the triangulated structures and makes sense also on the mixed level. We define an $\mathcal{A}$-linear map $\operatorname{gr}(\Theta): \mathfrak{K}(V) \rightarrow \mathfrak{K}\left(V^{\prime}\right)$ by the following requirement: If $A$ is a "character sheaf" on $V$ regarded as a mixed complex of pure weight 0 , then $\operatorname{gr}(\Theta)(A)=\operatorname{gr}(\Theta(A))$ where $\Theta(A)$ is regarded as a mixed complex on $V^{\prime}$ (with mixed structure defined by that of $A$ ). Note that $\operatorname{gr}(\Theta)(A)$ does not depend on the choice of mixed structure on $A$. If $\Theta^{\prime}: \mathcal{D}\left(V^{\prime}\right) \rightarrow \mathcal{D}\left(V^{\prime \prime}\right)$ is another functor like $\Theta$, then so is $\Theta^{\prime} \Theta$ and we have $\operatorname{gr}\left(\Theta^{\prime} \Theta\right)=\operatorname{gr}\left(\Theta^{\prime}\right) \operatorname{gr}(\Theta)$.

The previous discussion applies, in particular, to $V=Z_{J, D}$ or ${ }^{w} Z_{J, D}$ where $J \subset \mathbf{I}$ and $w \in{ }^{\epsilon(J)} \mathbf{W}$ and to the functors

$$
i_{J, w}^{*}: \mathcal{D}\left(Z_{J, D}\right) \rightarrow \mathcal{D}\left({ }^{w} Z_{J, D}\right), i_{J, w !}: \mathcal{D}\left({ }^{w} Z_{J, D}\right) \rightarrow \mathcal{D}\left(Z_{J, D}\right)
$$

(see 36.3(a), 36.3(f)); the character sheaves on ${ }^{w} Z_{J, D}$ are by definition the simple perverse sheaves isomorphic to ones in ${ }^{w} \hat{Z}_{J, D}$; see 36.3 . Hence $\mathfrak{K}\left(Z_{J, D}\right), \mathfrak{K}\left({ }^{w} Z_{J, D}\right)$ are defined and the $\mathcal{A}$-linear maps

$$
\operatorname{gr}\left(i_{J, w}^{*}\right): \mathfrak{K}\left(Z_{J, D}\right) \rightarrow \mathfrak{K}\left({ }^{w} Z_{J, D}\right), \operatorname{gr}\left(i_{J, w !}\right): \mathfrak{K}\left({ }^{w} Z_{J, D}\right) \rightarrow \mathfrak{K}\left(Z_{J, D}\right)
$$

are defined. Since $i_{J, w}^{*} i_{J, w !}=1$, we have $\operatorname{gr}\left(i_{J, w}^{*}\right) \operatorname{gr}\left(i_{J, w !}\right)=1$.

Let $\mathfrak{K}^{\prime}\left(Z_{J, D}\right)=\bigoplus_{w \in \epsilon(J)} \mathbf{W} \mathfrak{K}\left({ }^{w} Z_{J, D}\right)$. Define an $\mathcal{A}$-linear map $\phi: \mathfrak{K}\left(Z_{J, D}\right) \rightarrow$ $\mathfrak{K}^{\prime}\left(Z_{J, D}\right)$ by $\phi=\bigoplus_{w \in \epsilon(J)} \mathbf{W} g r\left(i_{J, w}^{*}\right)$.

From 36.3(e) we see that the matrix of $\phi$ is indexed by $\mathcal{I}_{J, D} \times \mathcal{I}_{J, D}$ and from $36.3(\mathrm{~b})$,(d) we see that this matrix is square and upper triangular (with 1 on diagonal) with respect to a suitable order on $\mathcal{I}_{J, D}$. In particular,

(a) $\phi: \mathfrak{K}\left(Z_{J, D}\right) \rightarrow \mathfrak{K}^{\prime}\left(Z_{J, D}\right)$ is an isomorphism.

Note that the inverse isomorphism restricted to $\mathfrak{K}\left({ }^{w} Z_{J, D}\right)$ is just $\operatorname{gr}\left(i_{J, w !}\right)$. Clearly,

(b) $\mathfrak{K}\left(Z_{J, D}\right)=\bigoplus_{w \in \epsilon(J)} \mathbf{W}^{w} \mathfrak{K}\left(Z_{J, D}\right)$, where ${ }^{w} \mathfrak{K}\left(Z_{J, D}\right)=\phi^{-1}\left(\mathfrak{K}\left({ }^{w} Z_{J, D}\right)\right)$.

We have a partition $\mathcal{I}_{J, D}=\bigsqcup_{\mathfrak{k} \in \mathbf{W} \backslash \mathfrak{s}} \mathcal{I}_{J, D}^{\mathfrak{k}}$ where $\mathbf{W} \backslash \mathfrak{\underline { \mathfrak { s } }}$ is the set of $\mathbf{W}$-orbits on $\underline{\mathfrak{s}}$ and $\mathcal{I}_{J, D}^{\mathfrak{k}}$ consists of those $A \in \mathcal{I}_{J, D}$ such that $A \in \hat{Z}_{J, D}^{\mathcal{L}}$ for some $\mathcal{L} \in \mathfrak{s}$ whose isomorphism class is in $\mathfrak{k}$. We have

(c) $\mathfrak{K}\left(Z_{J, D}\right)=\bigoplus_{\mathfrak{k} \in \mathbf{W} \backslash \underline{\mathfrak{s}}} \mathfrak{K}^{\mathfrak{k}}\left(Z_{J, D}\right)$

where $\mathfrak{K}^{\mathfrak{k}}\left(Z_{J, D}\right)$ is the $\mathcal{A}$-submodule of $\mathfrak{K}\left(Z_{J, D}\right)$ generated by $\mathcal{I}_{J, D}^{\mathfrak{k}}$. From definitions we see that each $\mathfrak{K}\left({ }^{w} Z_{J, D}\right)$ and each ${ }^{w} \mathfrak{K}\left(Z_{J, D}\right)$ has a natural direct sum decomposition indexed by $\mathbf{W} \backslash \mathfrak{s}$ analogous to (c). It follows that the decompositions (b), (c) are compatible in the sense that

(d) $\mathfrak{K}\left(Z_{J, D}\right)=\bigoplus_{w, \mathfrak{k}}\left({ }^{w} \mathfrak{K}\left(Z_{J, D}\right) \cap \mathfrak{K}^{\mathfrak{k}}\left(Z_{J, D}\right)\right)$.

36.9. Let $J \subset J^{\prime} \subset \mathbf{I}$. As in $[\mathrm{L} 10,6.4,6.7(\mathrm{~b})]$ we see that $\mathfrak{f}_{J, J^{\prime}}, \mathfrak{e}_{J, J^{\prime}}$ restrict to functors $\mathcal{D}^{c s}\left(Z_{J, D}\right) \rightarrow \mathcal{D}^{c s}\left(Z_{J^{\prime}, D}\right), \mathcal{D}^{c s}\left(Z_{J^{\prime}, D}\right) \rightarrow \mathcal{D}^{c s}\left(Z_{J, D}\right)$. Hence the $\mathcal{A}$-linear maps $\operatorname{gr}\left(\mathfrak{f}_{J, J^{\prime}}\right): \mathfrak{K}\left(Z_{J, D}\right) \rightarrow \mathfrak{K}\left(Z_{J^{\prime}, D}\right), \operatorname{gr}\left(\mathfrak{e}_{J, J^{\prime}}\right): \mathfrak{K}\left(Z_{J^{\prime}, D}\right) \rightarrow \mathfrak{K}\left(Z_{J, D}\right)$ are defined as in 36.8; we denote them again by $\mathfrak{f}_{J, J^{\prime}}, \mathfrak{e}_{J, J^{\prime}}$. The identities 36.4 (b) continue to hold for these linear maps. We define a group homomorphism $\mathfrak{D}: \mathfrak{K}\left(Z_{J, D}\right) \rightarrow \mathfrak{K}\left(Z_{J, D}\right)$ by 
$\mathfrak{D}\left(v^{m} A\right)=v^{-m} A^{*}$ where $A^{*} \in \mathcal{I}_{J, D}$ is isomorphic to the Verdier dual of $A \in \mathcal{I}_{J, D}$. From 36.4(a) we deduce

$$
\mathfrak{D F}_{J, J^{\prime}}=v^{-2 a} \mathfrak{f}_{J, J^{\prime}} \mathfrak{D}: \mathfrak{K}\left(Z_{J, D}\right) \rightarrow \mathfrak{K}\left(Z_{J^{\prime}, D}\right)
$$

with $a$ as in 36.4(a).

If $J \subset J^{\prime}, \mathfrak{c}, \mathfrak{d}, a$ are as in 36.4 , then for $A \in \mathcal{I}_{J, D}$ we have

$$
\begin{aligned}
\mathfrak{f}_{J, J^{\prime}} A & =\sum_{A_{1} \in \mathcal{I}_{J^{\prime}, D}} x_{A, A_{1}} A_{1}, \\
x_{A, A_{1}} & =\sum_{j}(-v)^{j}\left(\text { multiplicity of } A_{1} \text { in }{ }^{p} H^{j-a}\left(\mathfrak{d}_{!} \mathfrak{c}^{*} A[a]\right)\right) \in \mathcal{A} .
\end{aligned}
$$

(Indeed, if we regard $A$ as a pure perverse sheaf of weight 0 , then $\mathfrak{c}^{*} A[a]$ is a pure perverse sheaf of weight $a$ (since $\mathfrak{c}$ is an affine space bundle with fibres of dimension $a)$; by [De, 6.2.6] applied to the proper morphism $\mathfrak{d}, \mathfrak{d} \mathfrak{c}^{*} A[a]$ is a pure complex of weight $a$. From [BBD, 5.4.1], we see that ${ }^{p} H^{j}\left(\mathfrak{d} ! \mathfrak{c}^{*} A\right)={ }^{p} H^{j-a}\left(\mathfrak{d} ! \mathfrak{c}^{*} A[a]\right)$ is pure of weight $(j-a)+a=j$.

Let $\xi \mapsto \bar{\xi}$ be the group homomorphism $\mathcal{A} \rightarrow \mathcal{A}$ given by $v^{m} \mapsto v^{-m}$ for $m \in \mathbf{Z}$. Since $\mathfrak{c}^{*} A[a]$ is a pure perverse sheaf, we have (using the relative hard Lefschetz theorem [BBD, 5.4.10]):

$$
\begin{aligned}
& \bar{x}_{A, A_{1}}=\sum_{j}(-v)^{-j}\left(\text { multiplicity of } A_{1} \text { in }{ }^{p} H^{j-a}\left(\mathfrak{d}_{!} \mathfrak{c}^{*} A[a]\right)\right) \\
& =\sum_{j}(-v)^{-j}\left(\text { multiplicity of } A_{1} \text { in }{ }^{p} H^{-j+a}\left(\mathfrak{d} ! \mathfrak{c}^{*} A[a]\right)\right) \\
& =\sum_{j^{\prime}}(-v)^{j^{\prime}-2 a}\left(\text { multiplicity of } A_{1} \text { in }{ }^{p} H^{j^{\prime}-a}\left(\mathfrak{d}_{!} \mathfrak{c}^{*} A[a]\right)\right)=v^{-2 a} x_{A, A_{1}} .
\end{aligned}
$$

Define a group homomorphism $\beta_{J}: \mathfrak{K}\left(Z_{J, D}\right) \rightarrow \mathfrak{K}\left(Z_{J, D}\right)$ by $\beta_{J}\left(v^{m} A\right)=v^{-m} A$ for $A \in \mathcal{I}_{J, D}, m \in \mathbf{Z}$. We see that $\beta_{J^{\prime}}\left(\mathfrak{f}_{J, J^{\prime}} A\right)=v^{-2 a} \mathfrak{f}_{J, J^{\prime}} A$ for any $A \in \mathcal{I}_{J, D}$. Hence for any $\xi \in \mathfrak{K}\left(Z_{J, D}\right)$ we have

$$
\beta_{J^{\prime}}\left(\mathfrak{f}_{J, J^{\prime}} \xi\right)=v^{-2 a} \mathfrak{f}_{J, J^{\prime}}\left(\beta_{J}(\xi)\right) .
$$

Define $\tilde{\mathfrak{D}}: \mathfrak{K}\left(Z_{J, D}\right) \rightarrow \mathfrak{K}\left(Z_{J, D}\right)$ by $\tilde{\mathfrak{D}}(\xi)=\mathfrak{D} \beta_{J}(\xi)=\beta_{J} \mathfrak{D}(\xi)$. Clearly, $\tilde{\mathfrak{D}}$ is $\mathcal{A}$ linear. Note that

(d) the maps $\mathfrak{f}_{J, J^{\prime}}, \mathfrak{e}_{J, J^{\prime}}, \beta_{J}$ are compatible with the decompositions of type 36.8(c) while $\mathfrak{D}, \tilde{\mathfrak{D}}$ map the summand corresponding to $\mathfrak{k}$ in $36.8(\mathrm{c})$ onto the summand corresponding to $\check{\mathfrak{k}}$. (Here $\check{\mathfrak{k}}$ is the image of $\mathfrak{k}$ under $\mathcal{L} \mapsto \check{\mathcal{L}}$; see 28.18.)

Let $J, D, D^{\prime}, b_{1}, b_{2}, \epsilon$ be as in 32.5 . By $32.21, b_{2 !} b_{1}^{*}: \mathcal{D}\left(Z_{J, D} \times Z_{\epsilon(J), D^{\prime}}\right) \rightarrow \mathcal{D}\left(Z_{J, D^{\prime} D}\right)$ restricts to a functor $\mathcal{D}^{c s}\left(Z_{J, D} \times Z_{\epsilon(J), D^{\prime}}\right) \rightarrow \mathcal{D}^{c s}\left(Z_{J, D^{\prime} D}\right)$, where the character sheaves on $Z_{J, D} \times Z_{\epsilon(J), D^{\prime}}$ are by definition complexes of the form $A \otimes A^{\prime}$ with $A \in \hat{Z}_{J, D}, A^{\prime} \in \hat{Z}_{\epsilon(J), D^{\prime}}$. Hence the $\mathcal{A}$-linear map $\operatorname{gr}\left(b_{2 !} b_{1}^{*}\right): \mathfrak{K}\left(Z_{J, D} \times Z_{\epsilon(J), D^{\prime}}\right) \rightarrow$ $\mathfrak{K}\left(Z_{J, D^{\prime} D}\right)$ or equivalently $\mathfrak{K}\left(Z_{J, D}\right) \otimes_{\mathcal{A}} \mathfrak{K}\left(Z_{\epsilon(J), D^{\prime}}\right) \rightarrow \mathfrak{K}\left(Z_{J, D^{\prime} D}\right)$ is well defined. (We have canonically $\mathfrak{K}\left(Z_{J, D} \times Z_{\epsilon(J), D^{\prime}}\right)=\mathfrak{K}\left(Z_{J, D}\right) \otimes_{\mathcal{A}} \mathfrak{K}\left(Z_{\epsilon(J), D^{\prime}}\right)$.) We write $\xi * \xi^{\prime}$ instead of $\operatorname{gr}\left(b_{2 !} b_{1}^{*}\right)\left(\xi \otimes \xi^{\prime}\right)$ where $\xi \in \mathfrak{K}\left(Z_{J, D}\right), \xi^{\prime} \in \mathfrak{K}\left(Z_{\epsilon(J), D^{\prime}}\right)$. For $\mathfrak{k}, \mathfrak{k}^{\prime} \in \mathbf{W} \backslash \mathfrak{s}$ and $\xi \in \mathfrak{K}^{\mathfrak{k}}\left(Z_{J, D}\right), \xi^{\prime} \in \mathfrak{K}^{\mathfrak{k}^{\prime}}\left(Z_{\epsilon(J), D^{\prime}}\right)$, we have $\xi * \xi^{\prime} \in \mathfrak{K}^{\mathfrak{k}}\left(Z_{J, D^{\prime} D}\right)$ if $\mathfrak{k}^{\prime}=\underline{D}(\mathfrak{k})$ and $\xi * \xi^{\prime}=0$ if $\mathfrak{k}^{\prime} \neq \underline{D}(\mathfrak{k})$ (see 32.6(a),(b)).

From 28.19 we see that $\partial_{!}: \mathcal{D}\left(Z_{J, D}\right) \rightarrow \mathcal{D}\left(Z_{\epsilon(J), D^{-1}}\right)$ restricts to an equivalence of categories $\mathcal{D}^{c s}\left(Z_{J, D}\right) \rightarrow \mathcal{D}^{c s}\left(Z_{\epsilon(J), D^{-1}}\right)$. Hence the $\mathcal{A}$-linear isomorphism 
$\operatorname{gr}\left(\partial_{!}\right): \mathfrak{K}\left(Z_{J, D}\right) \rightarrow \mathfrak{K}\left(Z_{\epsilon(J), D^{-1}}\right)$ is well defined; we denote it again by $\partial$. Now the composition $\mathfrak{K}\left(Z_{J, D}\right) \stackrel{\partial}{\rightarrow} \mathfrak{K}\left(Z_{\epsilon(J), D^{-1}}\right) \stackrel{\partial}{\rightarrow} \mathfrak{K}\left(Z_{J, D}\right)$ is the identity map.

In the setup of $36.5, \mathfrak{f}_{J, w, J^{\prime}, w^{\prime}}$ restricts to a functor $\mathcal{D}^{c s}\left({ }^{w} Z_{J, D}\right) \rightarrow \mathcal{D}^{c s}\left(w^{\prime} Z_{J^{\prime}, D}\right)$. (We use the analogous statement for $\mathfrak{f}_{J, J^{\prime}}$, as above, together with 36.5(a), 36.3(a), $36.3(\mathrm{f})$.) Hence the $\mathcal{A}$-linear map $\operatorname{gr}\left(\mathfrak{f}_{J, w, J^{\prime}, w^{\prime}}\right): \mathfrak{K}\left({ }^{w} Z_{J, D}\right) \rightarrow \mathfrak{K}\left(w^{\prime} Z_{J^{\prime}, D}\right)$ is well defined. We denote it again by $\mathfrak{f}_{J, w, J^{\prime}, w^{\prime}}$. Let $\mathfrak{f}_{J, J^{\prime}}^{\prime}: \mathfrak{K}^{\prime}\left(Z_{J, D}\right) \rightarrow \mathfrak{K}^{\prime}\left(Z_{J^{\prime}, D}\right)$ be the unique $\mathcal{A}$-linear map such that $p r_{w^{\prime}} \mathfrak{f}_{J, J^{\prime}}^{\prime} p r_{w}=\mathfrak{f}_{J, w, J^{\prime}, w^{\prime}}$ for any $w, w^{\prime}$ as above. (Here $p r_{w}$ is the projection of $\mathfrak{K}^{\prime}\left(Z_{J^{\prime}, D}\right)$ onto the direct summand $\mathfrak{K}\left({ }^{w} Z_{J, D}\right)$.) From 36.5 (a) we see that for $x \in \mathfrak{K}\left({ }^{w} Z_{J^{\prime}, D}\right)$ we have

$$
\mathfrak{f}_{J, J^{\prime}}^{\prime}=\phi \mathfrak{f}_{J, J^{\prime}} \phi^{-1} \text {. }
$$

We also have

$$
\partial \mathfrak{f}_{J, J^{\prime}}=\mathfrak{f}_{\epsilon(J), \epsilon\left(J^{\prime}\right)} \partial, \quad \partial \mathfrak{e}_{J, J^{\prime}}=\mathfrak{e}_{\epsilon(J), \epsilon\left(J^{\prime}\right)} \partial .
$$

Now the functor $\tau: \mathcal{D}\left(Z_{J, G^{0}}\right) \rightarrow \mathcal{D}$ (point) (see 36.4) restricts to a functor $\mathcal{D}^{c s}\left(Z_{J, G^{0}}\right)$ $\rightarrow \mathcal{D}^{c s}$ (point) where the point is regarded as having exactly one character sheaf, $\overline{\mathbf{Q}}_{l}$. Hence the $\mathcal{A}$-linear map $\operatorname{gr}(\tau): \mathfrak{K}\left(Z_{J, G^{0}}\right) \rightarrow \mathfrak{K}($ point $)=\mathcal{A}$ is well defined; we denote it again by $\tau$.

Let $p, i^{\prime}$ be as in 36.4. Then $p_{!} i^{\prime *}: \mathcal{D}\left(Z_{J, D} \times Z_{J, D}\right) \rightarrow \mathcal{D}$ (point) restricts to a functor $\mathcal{D}^{c s}\left(Z_{J, D} \times Z_{J, D}\right) \rightarrow \mathcal{D}^{c s}$ (point) where the character sheaves on $Z_{J, D} \times Z_{J, D}$ are by definition complexes of the form $A \otimes A^{\prime}$ with $A, A^{\prime} \in \hat{Z}_{J, D}$. Hence the $\mathcal{A}$ linear map $\operatorname{gr}\left(p_{i} i^{\prime *}\right): \mathfrak{K}\left(Z_{J, D} \times Z_{J, D}\right) \rightarrow \mathfrak{K}$ (point) $=\mathcal{A}$ or equivalently $\mathfrak{K}\left(Z_{J, D}\right) \otimes_{\mathcal{A}}$ $\mathfrak{K}\left(Z_{J, D}\right) \rightarrow \mathcal{A}$ is well defined. (We have canonically $\mathfrak{K}\left(Z_{J, D} \times Z_{J, D}\right)=\mathfrak{K}\left(Z_{J, D}\right) \otimes_{\mathcal{A}}$ $\mathfrak{K}\left(Z_{J, D}\right)$.) We write $\left(\xi: \xi^{\prime}\right)$ instead of $g r\left(p_{!} i^{\prime *}\right)\left(\xi \otimes \xi^{\prime}\right)$ where $\xi, \xi^{\prime} \in \mathfrak{K}\left(Z_{J, D}\right)$. From BBD, 5.1.14] we see that for $A, B \in \mathcal{I}_{J, D}$ we have $(A: B) \in \mathbf{Z}\left[v^{-1}\right]$ and from [L3, II,7.4] and its proof we see that the constant term of $(A: B)$ is $\delta_{A, B^{*}}$. Thus,

$$
(A: B) \in \delta_{A, B^{*}}+v^{-1} \mathbf{Z}\left[v^{-1}\right] \text {. }
$$

We show that

(h) if $\xi \in \mathfrak{K}\left(Z_{J, D}\right),(\xi: \tilde{\mathfrak{D}}(\xi))=0$, then $\xi=0$.

Assume that $\xi \neq 0$. We have $\xi=\sum_{A \in \mathcal{I}_{\text {J } D}, m \in \mathbf{Z}} g_{A, m} v^{m} A$ where $g_{A, m} \in \mathbf{Z}$ is zero for all but finitely many $A, m$. We can find $e \in \mathbf{Z}$ such that $g_{A, e} \neq 0$ for some $A \in \mathcal{I}_{J, D}$ and $g_{A, m}=0$ for all $m>e$ and all $A \in \mathcal{I}_{J, D}$. Then

$$
(\xi: \tilde{\mathfrak{D}}(\xi))=\sum_{A_{1}, A, m_{1}, m} g_{A_{1}, m_{1}} g_{A, m} v^{m_{1}+m}\left(A_{1}: A^{*}\right) .
$$

By (g) this equals $\sum_{A} g_{A, e}^{2} v^{2 e}+$ an element in $v^{2 e-1} \mathbf{Z}\left[v^{-1}\right]$. This is nonzero since $\sum_{A} g_{A, e}^{2} \in \mathbf{Z}_{>0}$. This proves (h).

We show that

(i) if $\mathfrak{k}, \mathfrak{k}^{\prime} \in \mathbf{W} \backslash \underline{\mathfrak{s}}$ and $\mathfrak{k}^{\prime} \neq \check{\mathfrak{k}}$, then $\left(\mathfrak{K}^{\mathfrak{k}}\left(Z_{J, D}\right): \mathfrak{K}^{\mathfrak{k}^{\prime}}\left(Z_{J, D}\right)\right)=0$.

Let $A \in \hat{Z}_{J, D}^{\mathcal{L}}, B \in \hat{Z}_{J, D}^{\mathcal{L}_{1}}$ where $\mathcal{L}, \mathcal{L}_{1} \in \mathfrak{s}$. It is enough to show that

if $H_{c}^{j}\left(Z_{J, D}, A \otimes B\right) \neq 0$ for some $j$, then the isomorphism class of $\mathcal{L}_{1}$ is in the $\mathbf{W}$-orbit of the isomorphism class of $\check{\mathcal{L}}$.

From our assumption we have $A * \partial_{!}(B) \neq 0$. We have $A \dashv \bar{K}_{J, D}^{\bar{s}, \mathcal{L}}$ as in 28.13(v) and similarly $B \dashv \bar{K}_{J, D}^{\bar{s}^{\prime}, \mathcal{L}_{1}}$. Hence $\partial_{!} B \dashv \bar{K}_{\epsilon(J), D^{-1}}^{\bar{s}^{\prime}, \mathcal{L}^{\prime \prime}}$ where $\mathcal{L}^{\prime \prime}=\left(\underline{D}^{-1}\right)^{*} \check{\mathcal{L}}$, see 28.19. We 
then have $\bar{K}_{J, D}^{\bar{s}, \mathcal{L}} * \bar{K}_{\epsilon(J), D^{-1}}^{\bar{s}^{\prime}, \mathcal{L}^{\prime \prime}} \neq 0$. Using $32.6\left(\right.$ a) we see that $\mathcal{L}, \mathcal{L}_{1}$ have the required property. This proves (i).

We show that $(\mathrm{j})$ if $w, w^{\prime} \in \mathbf{W}$ and $w \neq w^{\prime}$, then $\left({ }^{w} \mathfrak{K}\left(Z_{J, D}\right):{ }^{w^{\prime}} \mathfrak{K}\left(Z_{J, D}\right)\right)=0$.

It is enough to show that if $A \in \mathcal{D}^{c s}\left({ }^{w} Z_{J, D}\right), A^{\prime} \in \mathcal{D}^{c s}\left({ }^{\prime} Z_{J, D}\right)$, then

$$
p ! i^{*}\left(i_{J, w !} A \otimes i_{J, w^{\prime} !} A^{\prime}\right)=0
$$

with $p, i^{\prime}$ as in 36.4. It is enough to show that $\left(i_{J, w} \times i_{J, w^{\prime}}\right) ! i^{\prime *}\left(A \otimes A^{\prime}\right)=0$. This follows from the fact that ${ }^{w} Z_{J, D} \cap w^{\prime} Z_{J, D}=\emptyset$.

We show that

(k) if $w \in{ }^{\epsilon(J)} \mathbf{W}, w \neq 1$, then $\tau\left({ }^{w} \mathfrak{K}\left(Z_{J, G^{0}}\right)\right)=0$.

Since ${ }^{w} Z_{J, G^{0}},{ }^{1} Z_{J, G^{0}}$ are disjoint, we have $i_{J, 1}^{*} i_{J, w !}=0$, hence $i^{*} i_{J, w !}=0$ with $i$ as in 36.4 ; ( $\mathrm{k}$ ) follows.

From the definitions we have (l) $\partial\left({ }^{w} \mathfrak{K}\left(Z_{J, D}\right)\right)={ }^{w^{-1}} \mathfrak{K}\left(Z_{\epsilon(J), D^{-1}}\right)$ for any $w \in{ }^{\epsilon(J)} \mathbf{W}$ such that $\epsilon^{-1}(\operatorname{Ad}(w) J)=J$. (For such $w$ we have $w^{-1} \in{ }^{J} \mathbf{W}$.)

36.10. Let

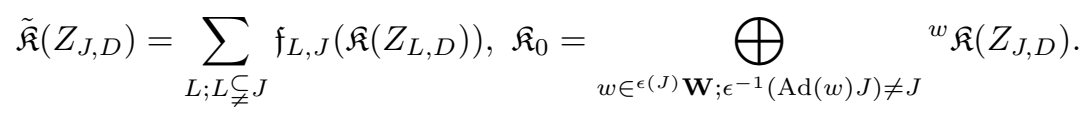

We show that

$$
\tilde{\mathfrak{K}}\left(Z_{J, D}\right)=\mathfrak{K}_{0} \oplus \bigoplus_{\substack{w \in \epsilon^{(J)} \mathbf{W} \\ \epsilon^{-1}(\operatorname{Ad}(w) J)=J}}\left(\tilde{\mathfrak{K}}\left(Z_{J, D}\right) \cap{ }^{w} \mathfrak{K}\left(Z_{J, D}\right)\right) .
$$

Setting

$$
\tilde{\mathfrak{K}}^{\prime}\left(Z_{J, D}\right)=\sum_{L ; L \varsubsetneqq J} \mathfrak{f}_{L, J}^{\prime}\left(\mathfrak{K}^{\prime}\left(Z_{L, D}\right)\right), \mathfrak{K}_{0}^{\prime}=\bigoplus_{w \in(J)} \bigoplus_{\mathbf{W} ; \epsilon^{-1}(\operatorname{Ad}(w) J) \neq J} \mathfrak{K}\left({ }^{w} Z_{J, D}\right)
$$

and using $36.9(\mathrm{e})$, we see that it is enough to show that

$$
\tilde{\mathfrak{K}}^{\prime}\left(Z_{J, D}\right)=\mathfrak{K}_{0} \oplus \bigoplus_{\substack{w \in \epsilon(J) \\ \epsilon^{-1}(\operatorname{Ad}(w) J)=J}}\left(\tilde{\mathfrak{K}}^{\prime}\left(Z_{J, D}\right) \cap \mathfrak{K}\left({ }^{w} Z_{J, D}\right)\right) .
$$

From 36.6(d), 36.6(e) we see that, if $w \in{ }^{\epsilon(J)} \mathbf{W}, \epsilon^{-1}(\operatorname{Ad}(w) J) \neq J$, then $A=$ $\mathfrak{f}_{J_{\infty}, J}^{\prime} A^{\prime}$ for some $A^{\prime} \in \mathfrak{K}\left({ }^{y} Z_{L^{\prime}, D}\right)$ and $J_{\infty} \neq J$. Hence

$$
\mathfrak{K}_{0}^{\prime} \subset \tilde{\mathfrak{K}}^{\prime}\left(Z_{J, D}\right) .
$$

Using again 36.6(d), 36.6(e) and also 36.4(b) (or rather its analogue for $\mathfrak{f}_{J, J^{\prime}}^{\prime}$ ) and (c), we see that

$$
\tilde{\mathfrak{K}}^{\prime}\left(Z_{J, D}\right)=\sum_{\substack{L ; L \subsetneq J \\ y \in \epsilon(\mathcal{}) \mathbf{W} \\ \epsilon^{-1}(\operatorname{Ad}(y) L)=L}} f_{L, J}^{\prime}\left(\mathfrak{K}\left({ }^{y} Z_{L, D}\right)\right)=\mathfrak{K}_{0}^{\prime}+\sum_{\substack{L ; L \subsetneq J \\ y \in \epsilon(\mathcal{}) \mathbf{W} \\ \epsilon^{-1}(\operatorname{Ad}(y) L)=L}} \mathfrak{f}_{L, J}^{\prime}\left(\mathfrak{K}\left({ }^{y} Z_{L, D}\right)\right) .
$$

Thus, (b) holds and (a) holds.

Let

$$
\overline{\mathfrak{K}}\left(Z_{J, D}\right)=\mathfrak{K}\left(Z_{J, D}\right) / \tilde{\mathfrak{K}}\left(Z_{J, D}\right) .
$$


For any $w \in{ }^{\epsilon(J)} \mathbf{W}$ such that $\epsilon^{-1}(\operatorname{Ad}(w) J)=J$, let ${ }^{w} \overline{\mathfrak{K}}\left(Z_{J, D}\right)$ be the image of $w_{\mathfrak{K}(}\left(Z_{J, D}\right)$ under the obvious map $\mathfrak{K}\left(Z_{J, D}\right) \rightarrow \overline{\mathfrak{K}}\left(Z_{J, D}\right)$. From (a) we see that

$$
\overline{\mathfrak{K}}\left(Z_{J, D}\right)=\bigoplus_{\substack{w \in(J) \\ w \\ \epsilon^{-1}(\operatorname{Ad}(w) J)=J}} w \overline{\mathfrak{K}}\left(Z_{J, D}\right) .
$$

For any $\mathfrak{k} \in \mathbf{W} \backslash \underline{\mathfrak{s}}$ let $\overline{\mathfrak{K}}^{\mathfrak{k}}\left(Z_{J, D}\right)$ be the image of $\mathfrak{K}^{\mathfrak{k}}\left(Z_{J, D}\right)$ under the obvious map $\mathfrak{K}\left(Z_{J, D}\right) \rightarrow \overline{\mathfrak{K}}\left(Z_{J, D}\right)$. From $36.9(\mathrm{~d})$ we see that $\tilde{\mathfrak{K}}\left(Z_{J, D}\right)$ is compatible with the decomposition $36.8(\mathrm{c})$. It follows that

$$
\overline{\mathfrak{K}}\left(Z_{J, D}\right)=\bigoplus_{\mathfrak{k} \in \mathbf{W} \backslash \underline{\mathfrak{s}}} \overline{\mathfrak{K}}^{\mathfrak{k}}\left(Z_{J, D}\right)
$$

Using 36.8(d) we see that that the decompositions (d),(e) are compatible in the sense that

$$
\overline{\mathfrak{K}}\left(Z_{J, D}\right)=\bigoplus_{w, \mathfrak{k}}\left({ }^{w} \overline{\mathfrak{K}}\left(Z_{J, D}\right) \cap \overline{\mathfrak{K}}^{\mathfrak{k}}\left(Z_{J, D}\right)\right) .
$$

Using 36.9(a), 36.9(c), we see that

$(\mathrm{g}) \mathfrak{D}, \beta_{J}, \tilde{\mathfrak{D}}: \mathfrak{K}\left(Z_{J, D}\right) \rightarrow \mathfrak{K}\left(Z_{J, D}\right)$ map $\tilde{\mathfrak{K}}\left(Z_{J, D}\right)$ into itself hence induces group homomorphisms $\overline{\mathfrak{K}}\left(Z_{J, D}\right) \rightarrow \overline{\mathfrak{K}}\left(Z_{J, D}\right)$ denoted again by $\mathfrak{D}, \beta_{J}, \tilde{\mathfrak{D}}$.

36.11. For $J^{\prime} \subset J \subset \mathbf{I}$ and $x \in \mathfrak{K}\left(Z_{J^{\prime}, D}\right), x^{\prime} \in \mathfrak{K}\left(Z_{\epsilon(J), D^{\prime}}\right)$, we show that

$$
\left(\mathfrak{f}_{J^{\prime}, J} x\right) * x^{\prime}=\mathfrak{f}_{J^{\prime}, J}\left(x * \mathfrak{e}_{\epsilon\left(J^{\prime}\right), \epsilon(J)} x^{\prime}\right) .
$$

Using successively 36.4(h), 36.9(f), 36.4(i), 36.4(c), 36.9(f), 36.4(h), 36.4(i), we see that, for any $x^{\prime \prime} \in \mathfrak{K}\left(Z_{J, D^{\prime} D}\right)$, we have

$$
\begin{aligned}
& \left(\left(\mathfrak{f}_{J^{\prime}, J} x\right) * x^{\prime}: x^{\prime \prime}\right)=\left(\left(\partial \mathfrak{f}_{J^{\prime}, J} x\right): x^{\prime} * \partial x^{\prime \prime}\right) \\
& =\left(\mathfrak{f}_{\epsilon\left(J^{\prime}\right), \epsilon(J)} \partial(x): x^{\prime} *\left(\partial x^{\prime \prime}\right)\right)=\left(\partial x: \mathfrak{e}_{\epsilon\left(J^{\prime}\right), \epsilon(J)}\left(x^{\prime} *\left(\partial x^{\prime \prime}\right)\right)\right. \\
& \left.=\left(\partial x: \mathfrak{e}_{\epsilon\left(J^{\prime}\right), \epsilon(J)}\left(x^{\prime}\right) * \mathfrak{e}_{\epsilon\left(J^{\prime}\right), \epsilon(J)}\left(\partial x^{\prime \prime}\right)\right)\right)=\left(\partial x: \mathfrak{e}_{\epsilon\left(J^{\prime}\right), \epsilon(J)} x^{\prime} * \partial \mathfrak{e}_{J^{\prime}, J} x^{\prime \prime}\right) \\
& =\left(x * \mathfrak{e}_{\epsilon\left(J^{\prime}\right), \epsilon(J)} x^{\prime}: \mathfrak{e}_{J^{\prime}, J} x^{\prime \prime}\right)=\left(\mathfrak{f}_{J^{\prime}, J}\left(x * \mathfrak{e}_{\epsilon\left(J^{\prime}\right), \epsilon(J)} x^{\prime}\right): x^{\prime \prime}\right) .
\end{aligned}
$$

Thus, if $\xi=\left(\mathfrak{f}_{J^{\prime}, J} x\right) * x^{\prime}-\mathfrak{f}_{J^{\prime}, J}\left(x * \mathfrak{e}_{\epsilon\left(J^{\prime}\right), \epsilon(J)} x^{\prime}\right)$, then $\left(\xi: x^{\prime \prime}\right)=0$ for any $x^{\prime \prime} \in$ $\mathfrak{K}\left(Z_{J, D^{\prime} D}\right)$. In particular, $(\xi: \tilde{\mathfrak{D}}(\xi))=0$. Using $36.9(\mathrm{~h})$ we see that $\xi=0$. This proves (a).

A similar argument shows that, if $x \in \mathfrak{K}\left(Z_{J, D}\right), x^{\prime} \in \mathfrak{K}\left(Z_{\epsilon\left(J^{\prime}\right), D^{\prime}}\right)$, then

$$
x *\left(\mathfrak{f}_{\epsilon\left(J^{\prime}\right), \epsilon(J)} x^{\prime}\right)=\mathfrak{f}_{J^{\prime}, J}\left(\mathfrak{e}_{J^{\prime}, J} x * x^{\prime}\right) .
$$

From (a),(b) we see that

$$
\tilde{\mathfrak{K}}\left(Z_{J, D}\right) * \mathfrak{K}\left(Z_{\epsilon(J), D^{\prime}}\right) \subset \tilde{\mathfrak{K}}\left(Z_{J, D^{\prime} D}\right), \mathfrak{K}\left(Z_{J, D}\right) * \tilde{\mathfrak{K}}\left(Z_{\epsilon(J), D^{\prime}}\right) \subset \tilde{\mathfrak{K}}\left(Z_{J, D^{\prime} D}\right) .
$$

It follows that $\mathfrak{K}\left(Z_{J, D}\right) \times \mathfrak{K}\left(Z_{\epsilon(J), D^{\prime}}\right) \rightarrow \mathfrak{K}\left(Z_{J, D^{\prime} D}\right), A, B \mapsto A * B$, induces an $\mathcal{A}$ bilinear pairing $\overline{\mathfrak{K}}\left(Z_{J, D}\right) \times \overline{\mathfrak{K}}\left(Z_{\epsilon(J), D^{\prime}}\right) \rightarrow \overline{\mathfrak{K}}\left(Z_{J, D^{\prime} D}\right)$. We denote it again by $A, B \mapsto$ $A * B$. The following result relates this bilinear pairing to the decompositions of type $36.10(\mathrm{~d})$.

For $w \in{ }^{\epsilon(J)} \mathbf{W}, w^{\prime} \in \epsilon^{\epsilon^{\prime} \epsilon(J)} \mathbf{W}$ such that $\epsilon^{-1}(\operatorname{Ad}(w) J)=J, \epsilon^{-1}\left(\operatorname{Ad}\left(w^{\prime}\right) \epsilon(J)\right)=$ $\epsilon(J)$, let $X_{w, w^{\prime}}$ be the set of all $y \in \epsilon^{\prime} \epsilon(J) \mathbf{W}$ such that $\epsilon^{-1} \epsilon^{\prime-1}(\operatorname{Ad}(y) J)=J$ and such that for some $Q \in \mathcal{P}_{J}, Q^{\prime} \in \mathcal{P}_{\epsilon(J)}, Q^{\prime \prime} \in \mathcal{P}_{\epsilon^{\prime} \epsilon(J)}$ we have

$$
\operatorname{pos}\left(Q^{\prime}, Q\right)=w, \operatorname{pos}\left(Q^{\prime \prime}, Q^{\prime}\right)=w^{\prime}, \operatorname{pos}\left(Q^{\prime \prime}, Q\right)=y .
$$


Then

$$
{ }^{w} \overline{\mathfrak{K}}\left(Z_{J, D}\right) *{ }^{w^{\prime}} \overline{\mathfrak{K}}\left(Z_{\epsilon(J), D^{\prime}}\right) \subset \bigoplus_{y \in X_{w, w^{\prime}}}{ }^{y} \overline{\mathfrak{K}}\left(Z_{J, D^{\prime} D}\right) .
$$

Indeed, using $36.7(\mathrm{a})$ we see that it is enough to show that

if ${ }^{w, w^{\prime}, y} Z_{0} \neq \emptyset$ (notation of 36.7), then $y \in X_{w, w^{\prime}}$.

This is immediate from definitions.

36.12. As a special case of the pairing $A, B \mapsto A * B$ in 36.11 we have an $\mathcal{A}$-bilinear pairing $\overline{\mathfrak{K}}\left(Z_{J, G^{0}}\right) \times \overline{\mathfrak{K}}\left(Z_{J, G^{0}}\right) \rightarrow \overline{\mathfrak{K}}\left(Z_{J, G^{0}}\right)$. This defines an associative algebra structure on $\overline{\mathfrak{K}}\left(Z_{J, G^{0}}\right)$ (not necessarily with 1 ).

Also as special cases of the pairing $A, B \mapsto A * B$ in 36.11 we have $\mathcal{A}$-bilinear pairings

$$
\overline{\mathfrak{K}}\left(Z_{J, G^{0}}\right) \times \overline{\mathfrak{K}}\left(Z_{J, D}\right) \rightarrow \overline{\mathfrak{K}}\left(Z_{J, D}\right), \quad \overline{\mathfrak{K}}\left(Z_{J, D}\right) \times \overline{\mathfrak{K}}\left(Z_{\epsilon(J), G^{0}}\right) \rightarrow \overline{\mathfrak{K}}\left(Z_{J, D}\right),
$$

which make $\overline{\mathfrak{K}}\left(Z_{J, D}\right)$ into a (not necessarily unital) $\left(\overline{\mathfrak{K}}\left(Z_{J, G^{0}}\right), \overline{\mathfrak{K}}\left(Z_{\epsilon(J), G^{0}}\right)\right)$-bimodule.

Let $\odot$ be the $\mathbf{W}$-orbit $\left\{\overline{\mathbf{Q}}_{l}\right\}$ in $\underline{\text { s. By }} 36.10(\mathrm{f})$ we have

$$
\overline{\mathfrak{K}}^{\bigcirc}\left(Z_{J, G^{0}}\right)=\bigoplus_{\substack{w \in \epsilon(J) \\ \epsilon^{-1}(\operatorname{Ad}(w) J)=J}}\left({ }^{w} \overline{\mathfrak{K}}\left(Z_{J, G^{0}}\right) \cap \overline{\mathfrak{K}}^{\bigodot}\left(Z_{J, G^{0}}\right)\right) .
$$

From $36.10(\mathrm{~g})$ we see that $\mathfrak{D}, \beta_{J}, \tilde{\mathfrak{D}}$ may be regarded as group homomorphisms $\overline{\mathfrak{K}}^{\bigcirc}\left(Z_{J, G^{0}}\right) \rightarrow \overline{\mathfrak{K}}^{\bigcirc}\left(Z_{J, G^{0}}\right)$.

36.13. For any $\mathcal{A}$-module $V$ we set $\underline{V}=\mathbf{Q}(v) \otimes_{\mathcal{A}} V$. If $V, V^{\prime}$ are $\mathcal{A}$-modules and $f: V \rightarrow V^{\prime}$ is $\mathcal{A}$-linear we denote again by $f$ the $\mathbf{Q}(v)$-linear map $\underline{V} \rightarrow \underline{V^{\prime}}$ such that $1 \otimes x \mapsto 1 \otimes f(x)$ for $x \in V$.

In particular, the $\mathbf{Q}(v)$-vector spaces $\underline{\mathfrak{K}}\left(Z_{J, D}\right), \underline{\tilde{\mathfrak{K}}}\left(Z_{J, D}\right)$ and $\underline{\overline{\mathfrak{K}}}\left(Z_{J, D}\right)$ are defined.

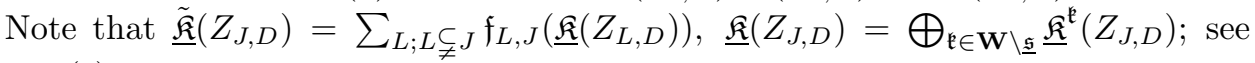
$36.8(\mathrm{c})$.

The symmetric bilinear form $(:): \mathfrak{K}\left(Z_{J, D}\right) \times \mathfrak{K}\left(Z_{J, D}\right) \rightarrow \mathcal{A}$ extends uniquely to a symmetric $\mathbf{Q}(v)$-bilinear form $\underline{\mathfrak{K}}\left(Z_{J, D}\right) \times \underline{\mathfrak{K}}\left(Z_{J, D}\right) \rightarrow \mathbf{Q}(v)$ denoted again by (:).

A vector subspace $E$ of $\underline{\mathfrak{K}}\left(Z_{J, D}\right)$ is said to be homogeneous if $E=\sum_{\mathfrak{k} \in \mathbf{W} \backslash \underline{\mathfrak{s}}} E^{\mathfrak{k}}$ where $E^{\mathfrak{k}}=E \cap \underline{\mathfrak{K}}^{\mathfrak{k}}\left(Z_{J, D}\right)$. For such $E$ we set $E^{\perp}=\left\{x \in \underline{\mathfrak{K}}\left(Z_{J, D}\right) ;(x: E)=0\right\}$. Then $E^{\perp}$ is homogeneous. We show that if, in addition, $E$ is stable under $\tilde{\mathfrak{D}}$, then: (a) $\underline{\mathfrak{K}}\left(Z_{J, D}\right)=E \oplus E^{\perp}$.

We first show that $E \cap E^{\perp}=0$. Assume that $x \in E \cap E^{\perp}$. Then $\tilde{f D}(x) \in E$ and $(x: \tilde{\mathfrak{D}}(x))=0$. We can find $\lambda \in \mathcal{A}-\{0\}$ such that $\lambda x \in \mathfrak{K}\left(Z_{J, D}\right)$. Then $(\lambda x, \tilde{\mathfrak{D}}(\lambda x))=0$. By 36.9 (h) we have $\lambda x=0$. Hence $x=0$ so that $E \cap E^{\perp}=0$. It remains to show that $\operatorname{dim}\left(E^{\perp}\right)^{\mathfrak{k}}+\operatorname{dim} E^{\mathfrak{k}}=\operatorname{dim} \underline{\mathfrak{K}}^{\mathfrak{k}}\left(Z_{J, D}\right)$ for any $\mathfrak{k}$. This is a consequence of the following statement:

(b) For any $\mathfrak{k}$, the form (:) on the finite dimensional vector space $\underline{\mathfrak{K}}^{\mathfrak{k}}\left(Z_{J, D}\right)+$ $\underline{\mathfrak{K}}^{\breve{\mathfrak{e}}}\left(Z_{J, D}\right)$ is nonsingular.

More generally, we show that for any finite dimensional subspace $E^{\prime}$ of $\underline{\mathfrak{K}}\left(Z_{J, D}\right)$ which is stable under $\tilde{\mathfrak{D}}$, the form (:) on $E^{\prime}$ is nonsingular. Let $x \in E^{\prime}$ be such that $\left(x: x^{\prime}\right)=0$ for any $x^{\prime} \in E^{\prime}$. In particular, we have $(x, \tilde{\mathfrak{D}}(x))=0$. As above, this implies that $x=0$. This proves (b), hence also (a). 
We set

$$
\underline{\mathfrak{K}}\left(Z_{J, D}\right)^{J}=\left\{\xi \in \underline{\mathfrak{K}}\left(Z_{J, D}\right) ; \mathfrak{e}_{H, J} \xi=0 \quad \forall H \varsubsetneqq J\right\} .
$$

We show:

(c)

$$
\underline{\tilde{\mathfrak{K}}}\left(Z_{J, D}\right)^{\perp}=\underline{\mathfrak{K}}\left(Z_{J, D}\right)^{J} .
$$

We have

$$
\begin{aligned}
& \underline{\tilde{\mathfrak{K}}}\left(Z_{J, D}\right)=\left\{x \in \underline{\mathfrak{K}}\left(Z_{J, D}\right) ;\left(x: \mathfrak{f}_{H, J} \underline{\mathfrak{K}}\left(Z_{H, D}\right)\right)=0 \quad \forall H \varsubsetneqq J\right\} \\
& =\left\{\xi \in \underline{\mathfrak{K}}\left(Z_{J, D}\right) ;\left(\mathfrak{e}_{H, J} x: \underline{\mathfrak{K}}\left(Z_{H, D}\right)\right)=0 \quad \forall H \varsubsetneqq J\right\} \\
& =\left\{\xi \in \underline{\mathfrak{K}}\left(Z_{J, D}\right) ; \mathfrak{e}_{H, J} x=0 \quad \forall H \varsubsetneqq J\right\},
\end{aligned}
$$

as required. (We have used that $\underline{\mathfrak{K}}\left(Z_{H, D}\right)^{\perp}=0$ which follows from (a), applied to $H$ instead of $J$.) This proves (c).

We show that

$$
\underline{\mathfrak{K}}\left(Z_{J, D}\right)=\underline{\tilde{\mathfrak{K}}}\left(Z_{J, D}\right) \oplus \underline{\mathfrak{K}}\left(Z_{J, D}\right)^{J} .
$$

This follows from (a),(c) using the fact that $\underline{\tilde{\mathfrak{K}}}\left(Z_{J, D}\right)$ is stable under $\tilde{\mathfrak{D}}$; see $36.10(\mathrm{~g})$.

From (d) we see that the second projection $\underline{\mathfrak{K}}\left(Z_{J, D}\right) \rightarrow \underline{\mathfrak{K}}\left(Z_{J, D}\right)^{J}$ induces an isomorphism $\underline{\mathfrak{K}}\left(Z_{J, D}\right) / \underline{\tilde{\mathfrak{K}}}\left(Z_{J, D}\right) \stackrel{\sim}{\longrightarrow} \underline{\mathfrak{K}}\left(Z_{J, D}\right)^{J}$, that is, an isomorphism

$$
\underline{\overline{\mathfrak{K}}}\left(Z_{J, D}\right) \stackrel{\sim}{\longrightarrow} \underline{\mathfrak{K}}\left(Z_{J, D}\right)^{J} .
$$

36.14. The $\mathcal{A}$-algebra structure on $\mathfrak{K}\left(Z_{J, G^{0}}\right)$ (resp. $\overline{\mathfrak{K}}\left(Z_{J, G^{0}}\right)$ ) given by $*$ extends to a $\mathbf{Q}(v)$-algebra structure on $\underline{\mathfrak{K}}\left(Z_{J, G^{0}}\right)$ (resp. $\underline{\overline{\mathfrak{K}}}\left(Z_{J, G^{0}}\right)$ ) denoted again by $*$. Moreover, $\underline{\tilde{\mathfrak{\kappa}}}\left(Z_{J, G^{0}}\right)$ is a two-sided ideal of $\underline{\mathfrak{K}}\left(Z_{J, G^{0}}\right)$ (see 36.11$)$. Note that $\underline{\mathfrak{K}}\left(Z_{J, D}\right)^{J}$ is also a two-sided ideal of $\underline{\mathfrak{K}}\left(Z_{J, G^{0}}\right)$, since $\mathfrak{e}_{H, J}: \underline{\mathfrak{K}}\left(Z_{J, G^{0}}\right) \rightarrow \underline{\mathfrak{K}}\left(Z_{H, G^{0}}\right)$ is an algebra homomorphism for any $H \subset J$ (see 36.4(c)). Since the two summands in the right-hand side of $36.13(\mathrm{~d})$ are two-sided ideals, they annihilate each other under the product $*$. We see also that the isomorphism 36.13(e) respects the algebra structures.

From 36.9(f) we see that the two summands in the right-hand side of $36.13(\mathrm{~d})$ are stable under $\partial: \underline{\mathfrak{K}}\left(Z_{J, G^{0}}\right) \rightarrow \underline{\mathfrak{K}}\left(Z_{J, G^{0}}\right)$ and from $36.4(\mathrm{~h})$ we have

$$
(x * y: \partial(z))=(\partial(x): y * z)
$$

for $x, y, z \in \underline{\mathfrak{K}}\left(Z_{J, G^{0}}\right)^{J}$.

Let $\mathcal{W}:=\left\{w \in{ }^{J} \mathbf{W} ; \operatorname{Ad}(w) J=J\right\}$. We show that

$$
\underline{\mathfrak{K}}\left(Z_{J, G^{0}}\right)^{J}=\bigoplus_{w \in \mathcal{W}}{ }^{w} \underline{\mathfrak{K}}\left(Z_{J, G^{0}}\right)^{J}
$$

where ${ }^{w} \underline{\mathfrak{K}}\left(Z_{J, G^{0}}\right)^{J}={ }^{w} \underline{\mathfrak{K}}\left(Z_{J, G^{0}}\right) \cap \underline{\mathfrak{K}}\left(Z_{J, G^{0}}\right)^{J}$. Let $x \in \underline{\mathfrak{K}}\left(Z_{J, G^{0}}\right)^{J}$. By $36.8(\mathrm{~b})$ we can write uniquely $x=\sum_{w \in J^{J} \mathbf{W}} x_{w}$ where $x_{w} \in{ }^{w} \underline{\mathfrak{K}}\left(Z_{J, G^{0}}\right)$. It is enough to show that $x_{w} \in \underline{\mathfrak{K}}\left(Z_{J, G^{0}}\right)^{J}$ (that is, $\left(y: x_{w}\right)=0$ for any $\left.y \in \underline{\tilde{\mathfrak{K}}}\left(Z_{J, G^{0}}\right)\right)$ for all $w$ and $x_{w}=0$ (that is, $\left(y^{\prime}: x_{w}\right)=0$ for any $\left.y^{\prime} \in \underline{\mathfrak{K}}\left(Z_{J, G^{0}}\right)\right)$ if $\operatorname{Ad}(w) J \neq J$. For $x_{w}, y$ as above we have $y=\sum_{w^{\prime} \in J^{J} \mathbf{W}} y_{w^{\prime}}$ with $y_{w^{\prime}} \in \bar{w}^{w^{\prime}} \underline{\underline{K}}\left(Z_{J, G^{0}}\right) \cap \underline{\tilde{\mathfrak{K}}}\left(Z_{J, G^{0}}\right)$; see $36.10(\mathrm{a})$. Using 36.9(j) twice, we have $\left(y: x_{w}\right)=\left(y_{w}: x_{w}\right)=\left(y_{w}: x\right)=0$, as required. Now assume that $w \in{ }^{J} \mathbf{W}, \operatorname{Ad}(w) J \neq J$ and $y^{\prime} \in \underline{\mathfrak{K}}\left(Z_{J, G^{0}}\right)$. By $36.8(\mathrm{~b})$ we have $y^{\prime}=\sum_{w^{\prime} \in{ }^{J} \mathbf{W}} y_{w^{\prime}}^{\prime}$ with $y_{w^{\prime}}^{\prime} \in w^{w^{\prime}} \underline{\underline{K}}\left(Z_{J, G^{0}}\right)$; moreover, by $36.10\left(\right.$ a) we have $y_{w}^{\prime} \in \underline{\tilde{\mathfrak{K}}}\left(Z_{J, G^{0}}\right)$. Using 36.9(j) twice, we have $\left(y^{\prime}: x_{w}\right)=\left(y_{w}^{\prime}: x_{w}\right)=\left(y_{w}^{\prime}: x\right)=0$, as required. This proves (b).

From definitions we see that 
(c) the decomposition (b) corresponds under 36.13(e) to the decomposition $\underline{\overline{\mathfrak{K}}}\left(Z_{J, G^{0}}\right)=\bigoplus_{w \in \mathcal{W}}{ }^{w} \underline{\overline{\mathfrak{K}}}\left(Z_{J, G^{0}}\right)($ see $36.10(d))$.

Under the isomorphism $36.13(\mathrm{e})$, the involution $\tilde{\mathfrak{D}}: \underline{\overline{\mathfrak{K}}}\left(Z_{J, G^{0}}\right) \rightarrow \underline{\overline{\mathfrak{K}}}\left(Z_{J, G^{0}}\right)$ corresponds to an involution $\tilde{\mathfrak{D}}^{\prime}: \underline{\mathfrak{K}}\left(Z_{J, G^{0}}\right)^{J} \rightarrow \underline{\mathfrak{K}}\left(Z_{J, G^{0}}\right)^{J}$; this is related to the involution $\tilde{\mathfrak{D}}: \underline{\mathfrak{K}}\left(Z_{J, G^{0}}\right) \rightarrow \underline{\mathfrak{K}}\left(Z_{J, G^{0}}\right)$ by $\tilde{\mathfrak{D}}^{\prime}(x)=\tilde{\tilde{\mathfrak{D}}}(x) \bmod \underline{\tilde{\mathfrak{K}}}\left(Z_{J, G^{0}}\right)$ for $x \in \underline{\mathfrak{K}}\left(Z_{J, G^{0}}\right)^{J}$. Hence for $x, x^{\prime} \in \underline{\mathfrak{K}}\left(Z_{J, G^{0}}\right)^{J}$ we have $\left(\tilde{\mathfrak{D}}^{\prime}(x): x^{\prime}\right)=\left(\tilde{\mathfrak{D}}(x): x^{\prime}\right)$. Using this and $36.9(\mathrm{~h})$ we see that

(d) if $x \in \underline{\mathfrak{K}}\left(Z_{J, G^{0}}\right)^{J}$ satisfies $(x: \tilde{\mathfrak{D}}(x))=0$, then $x=0$.

Note that $\underline{\mathfrak{K}}^{\bigcirc}\left(Z_{J, G^{0}}\right)$ is a subalgebra of $\underline{\mathfrak{K}}\left(Z_{J, G^{0}}\right)$. Hence

$$
\underline{\mathfrak{K}}^{J}:=\underline{\mathfrak{K}}^{\complement}\left(Z_{J, G^{0}}\right) \cap \underline{\mathfrak{K}}\left(Z_{J, G^{0}}\right)^{J}
$$

is a subalgebra of $\underline{\mathfrak{K}}\left(Z_{J, G^{0}}\right)^{J}$. From (b) and $36.8(\mathrm{~d})$ we see that

$$
\underline{\mathfrak{K}}^{J}=\bigoplus_{w \in \mathcal{W}}{ }^{w} \underline{\mathfrak{K}}^{J}
$$

where ${ }^{w} \underline{\mathfrak{K}}^{J}={ }^{w} \underline{\mathfrak{K}}^{\ominus}\left(Z_{J, G^{0}}\right) \cap \underline{\mathfrak{K}}^{J}$. Now $\partial: \underline{\mathfrak{K}}\left(Z_{J, G^{0}}\right)^{J} \rightarrow \underline{\mathfrak{K}}\left(Z_{J, G^{0}}\right)^{J}$ leaves $\underline{\mathfrak{K}}^{J}$ stable. Moreover, for $w \in \mathcal{W}$ we have

$$
\partial\left({ }^{w} \underline{\mathfrak{K}}^{J}\right)={ }^{w^{-1}} \underline{\mathfrak{K}}^{J} .
$$

(see 36.9(l)).

Note that $\mathcal{W}$ is the same as the set of all $w \in \mathbf{W}$ such that the corresponding permutation of the set of roots leaves stable the set of simple roots corresponding to elements of $J$. Hence $\mathcal{W}$ is a subgroup of $\mathbf{W}$.

36.15. By 36.4(f) we have $\left(x: x^{\prime}\right)=\left(x^{\prime}: x\right)=\tau\left(x * \partial\left(x^{\prime}\right)\right)$ for $x, x^{\prime} \in \underline{\mathfrak{K}}^{J}$. By $36.13(\mathrm{~b})$, the bilinear form (:) is nondegenerate on (the finite dimensional vector space) $\underline{\mathfrak{K}}^{J}$. Hence there is a unique vector $x_{0} \in \underline{\mathfrak{K}}^{J}$ such that $\left(x_{0}: x\right)=\tau(x)$ for all $x \in \underline{\mathfrak{K}}^{J}$. Hence for $x, x^{\prime} \in \underline{\mathfrak{K}}^{J}$ we have $\left(x_{0}: x * \partial\left(x^{\prime}\right)\right)=\tau\left(x * \partial\left(x^{\prime}\right)\right)=\left(x: x^{\prime}\right)$. Using 36.14 (a) we rewrite this as $\left(\partial\left(x_{0}\right) * x: x^{\prime}\right)=\left(x: x^{\prime}\right)$. (We use also that $\partial^{2}=1$ on $\underline{\mathfrak{K}}^{J}$.) Using the nondegeneracy of (:) on $\underline{\mathfrak{K}}^{J}$ we deduce $\partial\left(x_{0}\right) * x=x$ for all $x \in \underline{\mathfrak{K}}^{J}$. For $x, x^{\prime} \in \underline{\Omega}^{J}$ we have also $\left(\partial(x) * x^{\prime}: x_{0}\right)=\tau\left(\partial(x) * x^{\prime}\right)=\left(\partial(x): \partial\left(x^{\prime}\right)\right)=\left(x: x^{\prime}\right)$ (we use 36.4(g)). Using 36.14(a) we rewrite this as $\left(x: x^{\prime} * \partial\left(x_{0}\right)\right)=\left(x: x^{\prime}\right)$. Using the nondegeneracy of $(:)$ on $\underline{\mathfrak{K}}^{J}$ we deduce $x^{\prime} * \partial\left(x_{0}\right)=x^{\prime}$ for all $x^{\prime} \in \underline{\mathfrak{K}}^{J}$. We see that the algebra $\underline{\mathfrak{K}}^{J}$ has a unit element, namely $\mathbf{1}=\partial\left(x_{0}\right)$. By $36.14(\mathrm{e})$ we have $x_{0}=\sum_{w \in \mathcal{W}} x_{0}^{w}$ where $x_{0}^{w} \in{ }^{w} \underline{\underline{K}}^{J}$. For any $x \in \underline{\underline{K}}^{J}$ we have similarly $x=\sum_{w \in \mathcal{W}} x^{w}$ where $x^{w} \in{ }^{w} \underline{\mathfrak{K}}^{J}$. From $36.9(\mathrm{k})$ we see that $\tau(x)=\tau\left(x^{1}\right)$. Hence $\left(x_{0}: x^{1}\right)=$ $\tau\left(x^{1}\right)=\tau(x)=\left(x_{0}: x\right)$. Using 36.9(j) we see that $\left(x_{0}: x^{1}\right)=\left(x_{0}^{1}: x^{1}\right)=\left(x_{0}^{1}: x\right)$ hence $\left(x_{0}^{1}: x\right)=\left(x_{0}: x\right)$. Using the nondegeneracy of $(:)$ on $\underline{\underline{K}}^{J}$ we deduce $x_{0}=x_{0}^{1}$, that is, $x_{0} \in{ }^{1} \underline{\mathfrak{K}}^{J}$. Using $36.14(\mathrm{f})$ we deduce that $1 \in{ }^{1} \underline{\underline{K}}^{J}$.

36.16. We preserve the setup of $36.14,36.15$. In this subsection we assume that $G=G^{0}$ is a symplectic group $S p_{2 n}(\mathbf{k})(n \geq 1)$ and we describe in this case the structure of the algebra $\mathfrak{K}^{J}$. (The proofs, which depend on results in this and future sections, will be given elsewhere.) We have $\mathbf{I}=\left\{s_{1}, s_{2}, \ldots, s_{n}\right\}$ where $s_{i} s_{i+1}$ has order 3 if $i=1,2, \ldots, n-2$ and order 4 if $i=n-1$; we have $s_{i} s_{j}=s_{j} s_{i}$ if $|i-j| \geq 2$.

(i) We have $\underline{\mathfrak{K}}^{J}=0$ unless 
(*) $J=\left\{s_{k+1}, s_{k+2}, \ldots, s_{n}\right\}$ with $0 \leq k \leq n$ such that $n-k=a^{2}+a$ for some $a \in \mathbf{N}$.

Now assume that $(*)$ holds. Then $\mathcal{W}$ is a Weyl group of type $B_{k}$ with standard generators $\sigma_{1}, \sigma_{2}, \ldots, \sigma_{k}$ where $\sigma_{i}=s_{i}$ for $1 \leq i<k$ and $\sigma_{k}$ is the unique element in the subgroup of $\mathbf{W}$ generated by $s_{k}, s_{k+1}, \ldots, s_{n}$ such that $\sigma_{k} \in \mathcal{W}-\{1\}$ (if $k \geq 1)$. Let $\tilde{l}: \mathcal{W} \rightarrow \mathbf{N}$ be the length function of the Weyl group $\mathcal{W}$.

(ii) For any $w \in \mathcal{W}$ we have $\operatorname{dim}\left({ }^{w} \underline{\mathfrak{K}}^{J}\right)=1$.

(iii) For any $i \in[1, k]$ there is a unique element $x \in{ }^{\sigma_{i}} \underline{\mathfrak{K}}^{J}-\{0\}$ such that $(x+\mathbf{1}) *(x-c \mathbf{1})=0$ for some $c \in v \mathbf{Z}[v]$; in fact, we have $c=v^{2}$ if $1 \leq i<k$ and $c=v^{4 a+2}$ if $i=k$. We set $x=t_{i}$.

(iv) For any $w \in \mathcal{W}$ there is a unique element $t_{w} \in{ }^{w} \underline{\underline{K}}^{J}-\{0\}$ such that the following hold: $t_{\sigma_{i}}=t_{i}$ for $i \in[1, k] ; t_{w} * t_{w^{\prime}}=t_{w^{\prime} w}$ if $w, w^{\prime} \in \mathcal{W}, \tilde{l}\left(w^{\prime} w\right)=$ $\tilde{l}(w)+\tilde{l}\left(w^{\prime}\right)$.

We see that $\underline{\mathfrak{K}}^{J}$ is an Iwahori-Hecke algebra with not necessarily equal parameters. Similar results hold for other classical groups.

\section{A MACKEy TyPe FORMUla}

37.1. We fix a connected component $D$ of $G$. With notation in 26.1 , if $J, J^{\prime} \subset$ $\mathbf{I}, P \in \mathcal{P}_{J}, Q \in \mathcal{P}_{J^{\prime}}, u=\operatorname{pos}(P, Q) \in \mathbf{W}$, then $u \in{ }^{J} \mathbf{W}^{J^{\prime}}$. Setting $P^{Q}=(P \cap Q) U_{P}$, we have $P^{Q} \in \mathcal{P}_{J \cap \operatorname{Ad}(u) J^{\prime}}$.

For $K, K^{\prime} \subset \mathbf{I}$ and $u \in{ }^{K} \mathbf{W}^{K^{\prime}}$ let

$$
\begin{aligned}
\Upsilon_{u}= & \left\{\left(X, Y, g\left(U_{X} \cap U_{Y}\right)\right) ; X \in \mathcal{P}_{K \cap \operatorname{Ad}(u) K^{\prime}}, Y \in \mathcal{P}_{K^{\prime} \cap \operatorname{Ad}\left(u^{-1}\right) K},\right. \\
& \left.g\left(U_{X} \cap U_{Y}\right) \in D /\left(U_{X} \cap U_{Y}\right), \operatorname{pos}(X, Y)=u\right\} .
\end{aligned}
$$

We have a diagram

$$
Z_{K \cap \operatorname{Ad}(u) K^{\prime}, D} \stackrel{\mathfrak{j}}{\leftarrow} \Upsilon_{u} \stackrel{\mathfrak{h}}{\rightarrow} Z_{K^{\prime} \cap \operatorname{Ad}\left(u^{-1}\right) K, D}
$$

where $\mathfrak{j}\left(X, Y, g\left(U_{X} \cap U_{Y}\right)\right)=\left(X, g U_{X}\right), \mathfrak{h}\left(X, Y, g\left(U_{X} \cap U_{Y}\right)\right)=\left(Y, g U_{Y}\right)$. Set

$$
\Phi_{u}=\mathfrak{h}_{\mathbf{j}^{*}}: \mathcal{D}\left(Z_{K \cap \operatorname{Ad}(u) K^{\prime}, D}\right) \rightarrow \mathcal{D}\left(Z_{K^{\prime} \cap \operatorname{Ad}\left(u^{-1}\right) K, D}\right) .
$$

Proposition 37.2. Let $K, K^{\prime}, J \subset \mathbf{I}$ be such that $K \subset J, K^{\prime} \subset J$. Let $A^{\prime} \in$ $\mathcal{D}\left(Z_{K, D}\right)$. We set $\mathfrak{B}=\mathfrak{e}_{K^{\prime}, J} \mathfrak{f}_{K, J} A^{\prime} \in \mathcal{D}\left(Z_{K^{\prime}, D}\right)$. For any $u \in{ }^{K} \mathbf{W}^{K^{\prime}} \cap \mathbf{W}_{J}$ we set $\mathfrak{B}_{u}=\mathfrak{f}_{K^{\prime} \cap \operatorname{Ad}\left(u^{-1}\right) K, K^{\prime}} \Phi_{u} \mathfrak{e}_{K \cap \operatorname{Ad}(u) K^{\prime}, K} A^{\prime} \in \mathcal{D}\left(Z_{K^{\prime}, D}\right)$ and $m_{u}=\operatorname{dim}\left(U_{P} \cap U_{R}\right) / U_{Q}$ where $P \in \mathcal{P}_{K}, R \in \mathcal{P}_{K^{\prime}}, \operatorname{pos}(P, R)=u$ and $Q=Q_{J, P}=Q_{J, R} \in \mathcal{P}_{J}$ (notation of 36.4). We have

$$
\mathfrak{B} \approx\left\{\mathfrak{B}_{u}\left[\left[-m_{u}\right]\right] ; u \in{ }^{K} \mathbf{W}^{K^{\prime}} \cap \mathbf{W}_{J}\right\},
$$

with $\approx$ as in 32.15.

We have a commutative diagram with a cartesian square

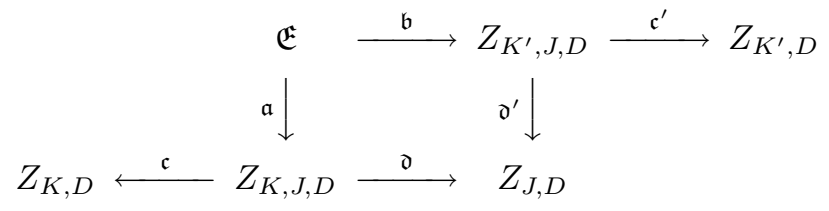

Here

$\mathfrak{E}=\left\{\left(P, R, g U_{Q}\right) ; P \in \mathcal{P}_{K}, R \in \mathcal{P}_{K^{\prime}}, g U_{Q} \in D / U_{Q}, Q=Q_{J, P}=Q_{J, R}\right\}$,

$\mathfrak{c}\left(P, g U_{Q}\right)=\left(P, g U_{P}\right), \mathfrak{o}\left(P, g U_{Q}\right)=\left(Q, g U_{Q}\right)$ with $Q=Q_{J, P}$, 
$\mathfrak{c}^{\prime}\left(R, g U_{Q}\right)=\left(R, g U_{R}\right), \mathfrak{d}^{\prime}\left(R, g U_{Q}\right)=\left(Q, g U_{Q}\right)$ with $Q=Q_{J, R}$,

$\mathfrak{a}\left(P, R, g U_{Q}\right)=\left(P, g U_{Q}\right), \mathfrak{b}\left(P, R, g U_{Q}\right)=\left(R, g U_{Q}\right)$.

We have

$$
\mathfrak{B}=\mathfrak{c}_{!}^{\prime} \mathfrak{d}^{\prime *} \mathfrak{d}_{!} \mathfrak{c}^{*} A^{\prime}=\mathfrak{c}_{!}^{\prime} \mathfrak{b}_{!} \mathfrak{a}^{*} \mathfrak{c}^{*} A^{\prime}=\left(\mathfrak{c}^{\prime} \mathfrak{b}\right)_{!}(\mathfrak{c a})^{*} A^{\prime}=\mathfrak{q} ! \mathfrak{p}^{*} A^{\prime}
$$

where $\mathfrak{q}=\mathfrak{c}^{\prime} \mathfrak{b}: \mathfrak{E} \rightarrow Z_{K^{\prime}, D}, \mathfrak{p}=\mathfrak{c a}: \mathfrak{E} \rightarrow Z_{K, D}$ are given by $\mathfrak{q}\left(P, R, g U_{Q}\right)=$ $\left(R, g U_{R}\right), \mathfrak{p}\left(P, R, g U_{Q}\right)=\left(P, g U_{P}\right)$. We have a partition

$$
\mathfrak{E}=\bigsqcup_{u \in{ }^{K} \mathbf{W}^{K^{\prime}} \cap \mathbf{W}_{J}} \mathfrak{E}_{u}
$$

where $\mathfrak{E}_{u}=\left\{\left(P, R, g U_{Q}\right) \in \mathfrak{E} ; \operatorname{pos}(P, R)=u\right\}$ is locally closed in $\mathfrak{E}$. Let $\mathfrak{p}_{u}=\left.\mathfrak{p}\right|_{\mathfrak{E}_{u}}$ : $\mathfrak{E}_{u} \rightarrow Z_{K, D}, \mathfrak{q}_{u}=\left.\mathfrak{q}\right|_{\mathfrak{E}_{u}}: \mathfrak{E}_{u} \rightarrow Z_{K^{\prime}}$. By 32.15 , we have

$$
\mathfrak{q} ! \mathfrak{p}^{*} A^{\prime} \approx\left\{\mathfrak{q}_{u} ! \mathfrak{p}_{u}^{*} A^{\prime} ; u \in{ }^{K} \mathbf{W}^{K^{\prime}} \cap \mathbf{W}_{J}\right\} .
$$

It remains to show that, for $u$ as above, we have

$$
\mathfrak{q}_{u} \mathfrak{p}_{u}^{*} A^{\prime}=\mathfrak{B}_{u}\left[\left[-m_{u}\right]\right]
$$

We have a commutative diagram

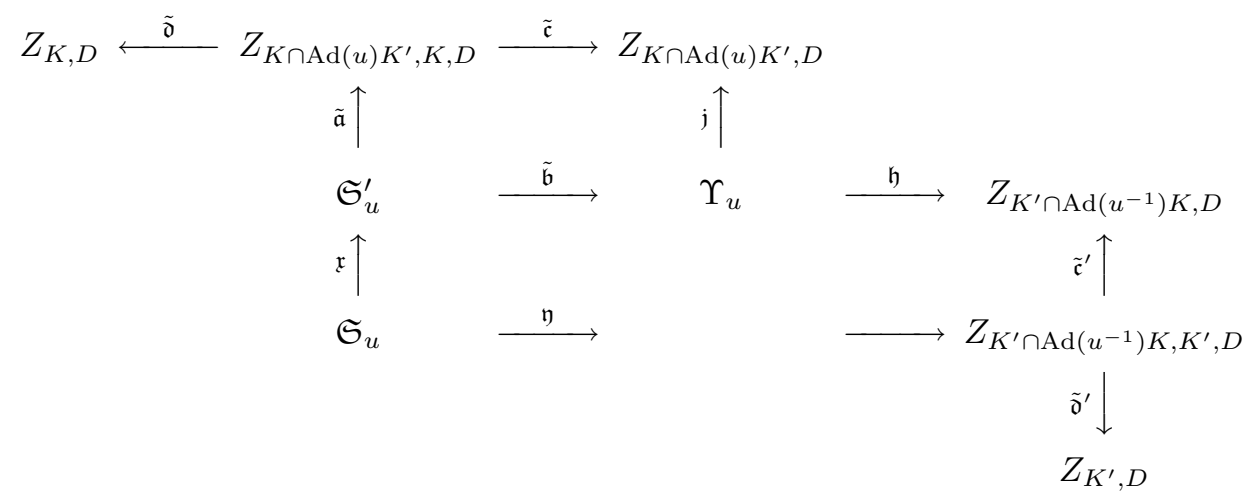

Here

$\tilde{\mathfrak{c}}\left(X, g U_{P}\right)=\left(X, g U_{X}\right), \tilde{\mathfrak{d}}\left(X, g U_{P}\right)=\left(P, g U_{P}\right)$,

$\tilde{\mathfrak{c}}^{\prime}\left(Y, g U_{R}\right)=\left(Y, g U_{Y}\right), \tilde{\mathfrak{d}}^{\prime}\left(Y, g U_{R}\right)=\left(R, g U_{R}\right)$,

$\mathfrak{S}_{u}^{\prime}, \tilde{\mathfrak{a}}, \tilde{\mathfrak{b}}$ are defined so that the square $(\tilde{\mathfrak{a}}, \tilde{\mathfrak{b}}, \tilde{\mathfrak{c}}, \mathfrak{j})$ is cartesian;

$\mathfrak{S}_{u}, \mathfrak{x}, \mathfrak{y}$ are defined so that the square $\left(\mathfrak{x}, \mathfrak{y}, \mathfrak{h} \tilde{\mathfrak{b}}, \tilde{\mathfrak{c}}^{\prime}\right)$ is cartesian.

Then

$$
\begin{aligned}
\mathfrak{S}_{u}= & \left\{\left(X, Y, g\left(U_{X} \cap U_{Y}\right), g^{\prime} U_{P}, g^{\prime \prime} U_{R}\right) ; X \in \mathcal{P}_{K \cap \operatorname{Ad}(u) K^{\prime}}, Y \in \mathcal{P}_{K^{\prime} \cap \operatorname{Ad}\left(u^{-1}\right) K},\right. \\
& g\left(U_{X} \cap U_{Y}\right) \in D /\left(U_{X} \cap U_{Y}\right), g^{\prime} U_{P} \in D / U_{P}, g^{\prime \prime} U_{R} \in D / U_{R}, \operatorname{pos}(X, Y)=u, \\
& \left.P=Q_{K, X}, R=Q_{K^{\prime}, Y}, g^{\prime} U_{X}=g U_{X}, g^{\prime \prime} U_{Y}=g U_{Y}\right\} .
\end{aligned}
$$

Set $\mathfrak{r}=\tilde{\mathfrak{d}}^{\prime} \mathfrak{y}: \mathfrak{S}_{u} \rightarrow Z_{K^{\prime}, D}, \mathfrak{s}=\tilde{\mathfrak{d}} \tilde{\mathfrak{a}} \mathfrak{x}: \mathfrak{S}_{u} \rightarrow Z_{K, D}$. Then

$$
\begin{aligned}
& \mathfrak{r}\left(X, Y, g\left(U_{X} \cap U_{Y}\right), g^{\prime} U_{P}, g^{\prime \prime} U_{R}\right)=\left(P, g^{\prime} U_{P}\right), \\
& \mathfrak{s}\left(X, Y, g\left(U_{X} \cap U_{Y}\right), g^{\prime} U_{P}, g^{\prime \prime} U_{R}\right)=\left(R, g^{\prime \prime} U_{R}\right) .
\end{aligned}
$$

We have

$$
\begin{aligned}
& \mathfrak{B}_{u}=\tilde{\mathfrak{d}}_{!}^{\prime} \tilde{\mathfrak{c}}^{\prime *} \mathfrak{h} ! \mathfrak{j}^{*} \tilde{\mathfrak{c}}_{!} \tilde{\mathfrak{d}}^{*} A^{\prime}=\tilde{\mathfrak{d}}_{!}^{\prime} \tilde{\mathfrak{c}}^{*} \mathfrak{h} ! \tilde{\mathfrak{b}}_{!} \tilde{\mathfrak{a}}^{*} \tilde{\mathfrak{d}}^{*} A^{\prime} \\
& =\tilde{\mathfrak{d}} ! \tilde{\mathfrak{c}}^{\prime *}(\mathfrak{h} \tilde{\mathfrak{b}}) !(\tilde{\mathfrak{d}} \tilde{\mathfrak{a}})^{*} A^{\prime}=\tilde{\mathfrak{d}} ! \mathfrak{y} ! \mathfrak{x}^{*}(\tilde{\mathfrak{d}} \tilde{\mathfrak{a}})^{*} A^{\prime}=(\tilde{\mathfrak{d}} \mathfrak{y}) !(\tilde{\mathfrak{d}} \tilde{\mathfrak{a}})^{*} A^{\prime}=\mathfrak{r} ! \mathfrak{s}^{*} A^{\prime}
\end{aligned}
$$


We show that $\mathfrak{q}_{u} ! \mathfrak{p}_{u}^{*} A^{\prime}=\mathfrak{r}_{!} \mathfrak{s}^{*} A^{\prime}\left[\left[-m_{u}\right]\right]$. We have a commutative diagram

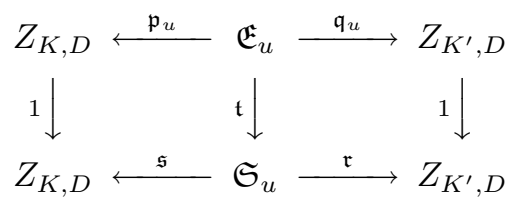

where $\mathfrak{t}\left(P, R, g U_{Q}\right)=\left(P^{R}, R^{P}, g\left(U_{P^{R}} \cap U_{R^{P}}\right), g U_{P}, g U_{R}\right)$ is well defined since $U_{Q} \subset$ $U_{P}, U_{Q} \subset U_{R}, U_{Q} \subset U_{P^{R}}, U_{Q} \subset U_{R^{P}}$. We continue the proof assuming that

(a) $\mathfrak{t}$ is an affine space bundle with fibres of dimension $m_{u}$.

For any $\tilde{A} \in \mathcal{D}\left(\mathfrak{S}_{u}\right)$ we have $\mathfrak{t}_{!} \mathbf{t}^{*}(\tilde{A})=\tilde{A}\left[\left[-m_{u}\right]\right]$. Hence

$$
\mathfrak{r}_{!} \mathfrak{s}^{*} A^{\prime}\left[\left[-m_{u}\right]\right]=\mathfrak{r}_{!} \mathfrak{t}_{!} t^{*} \mathfrak{s}^{*} A^{\prime}=(\mathfrak{r t})_{!}(\mathfrak{s t})^{*} A^{\prime}=\mathfrak{q}_{u} ! \mathfrak{p}_{u}^{*} A^{\prime}
$$

as required.

We prove (a). We only show that each fibre of $\mathfrak{t}$ is an affine space of dimension $m_{u}$. First we show that $\mathfrak{t}: \mathfrak{E}_{u} \rightarrow \mathfrak{S}_{u}$ is surjective. Assume that we are given $\left(X, Y, g_{0}\left(U_{X} \cap U_{Y}\right), g_{0}^{\prime} U_{P}, g_{0}^{\prime \prime} U_{R}\right) \in \mathfrak{S}_{u}$. Then $g_{0}, g_{0}^{\prime}, g_{0}^{\prime \prime} \in D, v^{\prime}=g_{0}^{-1} g_{0}^{\prime} \in U_{X}$, $v^{\prime \prime}=g_{0}^{-1} g_{0}^{\prime \prime} \in U_{Y}$. We must show that there exists $g \in D$ such that $g_{0} \in g\left(U_{X} \cap\right.$ $\left.U_{Y}\right), g_{0}^{\prime} \in g U_{P}, g_{0}^{\prime \prime} \in g U_{R}$. Setting $y=g^{-1} g_{0}$, we must show that there exists $y \in U_{X} \cap U_{Y}$ such that $y v^{\prime} \in U_{P}, y v^{\prime \prime} \in U_{R}$. We have $v^{\prime}=v_{1}^{\prime} v_{2}^{\prime}$ where $v_{1}^{\prime} \in U_{R} \cap P$, $v_{2}^{\prime} \in U_{P}$ and $v^{\prime \prime}=v_{1}^{\prime \prime} v_{2}^{\prime \prime}$ where $v_{1}^{\prime \prime} \in U_{P} \cap R, v_{2}^{\prime \prime} \in U_{R}$. Then $v_{1}^{\prime} \in U_{X} \cap U_{Y}, v_{1}^{\prime \prime} \in$ $U_{X} \cap U_{Y}$. Setting $y=\left(v_{1}^{\prime} v_{1}^{\prime \prime}\right)^{-1} \in U_{X} \cap U_{Y}$ we have $y v^{\prime}=v_{1}^{\prime \prime-1} v_{2}^{\prime} \in U_{P}$ and $y v^{\prime \prime}=v_{1}^{\prime \prime-1} v_{1}^{\prime-1} v_{1}^{\prime \prime} v_{2}^{\prime \prime} \in v_{1}^{\prime \prime-1} U_{R} v_{1}^{\prime \prime} U_{R}=U_{R}$, as desired.

It remains to show that, if $\left(P, R, g U_{Q}\right) \in \mathfrak{E}_{u}$, then

(b) $F=\left\{\left(P^{\prime}, R^{\prime}, g^{\prime} U_{Q^{\prime}}\right) \in E_{u} ; \mathfrak{t}\left(P^{\prime}, R^{\prime}, g^{\prime} U_{Q^{\prime}}\right)=\mathfrak{t}\left(P, R, g U_{Q}\right)\right\}$ is an affine space of dimension $m_{u}$.

For $\left(P^{\prime}, R^{\prime}, g^{\prime} U_{Q^{\prime}}\right) \in F$, both $P, P^{\prime}$ contain $P^{R}=\left(P^{\prime}\right)^{R^{\prime}}$ and have the same type, hence $P=P^{\prime}$. Similarly, $R=R^{\prime}, Q=Q^{\prime}$. Hence

$$
\begin{aligned}
& F \cong\left\{g^{\prime} U_{Q} ; g U_{P}=g^{\prime} U_{P}, g U_{R}=g^{\prime} U_{R}, g\left(U_{P^{R}} \cap U_{R^{P}}\right)=g^{\prime}\left(U_{P^{R}} \cap U_{R^{P}}\right)\right\} \\
& =\left\{g^{\prime} U_{Q} ; g^{-1} g^{\prime} \in U_{P} \cap U_{R} \cap U_{P^{R}} \cap U_{R^{P}}\right\} \\
& =\left\{g^{\prime} U_{Q} ; g^{-1} g^{\prime} \in U_{P} \cap U_{R}\right\} \cong\left(U_{P} \cap U_{R}\right) / U_{Q},
\end{aligned}
$$

and (b) follows. This completes the proof.

37.3. For any $n \in \mathbf{N}_{\mathbf{k}}^{*}$ we have a $G^{0} \times \mathbf{T}$-action on $Z_{\emptyset, D}$ given by

(a) $\left(g_{1}, t\right):\left(B, g U_{B}\right) \mapsto\left(g_{1} B g_{1}^{-1}, g_{1} g b^{-n} g_{1}^{-1} U_{g_{1} B g_{1}^{-1}}\right)$

where $b \in B$ is such that $f_{B}(t)=b U_{B}\left(f_{B}\right.$ as in 28.3). Let $u \in \mathbf{W}$. We consider another $G^{0} \times \mathbf{T}$-action on $Z_{\emptyset, D}$ given by

(b) $\left(g_{1}, t\right):\left(B, g U_{B}\right) \mapsto\left(g_{1} B g_{1}^{-1}, g_{1} g b_{1}^{-n} g_{1}^{-1} U_{g_{1} B g_{1}^{-1}}\right)$

where $b_{1} \in B$ is such that $f_{B}\left(u^{-1}(t)\right)=b_{1} U_{B}\left(f_{B}\right.$ as in 28.3).

Using 36.3 we see that a simple perverse sheaf on $Z_{\emptyset, D}$ is a character sheaf if and only if it is $G^{0} \times \mathbf{T}$-equivariant for the action (a) for some $n$ or equivalently if it is $G^{0} \times \mathbf{T}$-equivariant for the action (b) for some $n$.

Let $\Upsilon_{u, 0}$ be like $\Upsilon_{u}$ in 37.1 but with $K, K^{\prime}$ replaced by $\emptyset, \emptyset$. We have a diagram $Z_{\emptyset, D} \stackrel{\mathfrak{j}_{0}}{\longrightarrow} \Upsilon_{u, 0} \stackrel{\mathfrak{h}_{0}}{\longrightarrow} Z_{\emptyset, D}\left(\right.$ a special case of the diagram in 37.1 with $\left.K=K^{\prime}=\emptyset\right)$. Set $\Phi_{u}^{\emptyset}=\mathfrak{h}_{0 !} \mathfrak{j}_{0}^{*}: \mathcal{D}\left(Z_{\emptyset, D}\right) \rightarrow \mathcal{D}\left(Z_{\emptyset, D}\right)$. We show that

(c) $\Phi_{u}^{\emptyset}$ restricts to a functor $\mathcal{D}^{c s}\left(Z_{\emptyset, D}\right) \rightarrow \mathcal{D}^{c s}\left(Z_{\emptyset, D}\right)$. 
It is enough to show that if $A$ is a character sheaf on $Z_{\emptyset, D}$, then $\Phi_{u}^{\emptyset}(A) \in \mathcal{D}^{c s}\left(Z_{\emptyset, D}\right)$. Now $G^{0} \times \mathbf{T}$ acts on $\Upsilon_{u, 0}$ by

$\left(g_{1}, t\right):\left(B, B^{\prime}, g\left(U_{B} \cap U_{B^{\prime}}\right)\right) \mapsto\left(g_{1} B g_{1}^{-1}, g_{1} B^{\prime} g_{1}^{-1}, g_{1} g b^{-n} g_{1}^{-1}\left(U_{g_{1} B g_{1}^{-1}} \cap U_{g_{1} B g_{1}^{-1}}\right)\right)$

where $b \in B \cap B^{\prime}$ is such that $f_{B}(t)=b U_{B}\left(f_{B}\right.$ as in 28.3). Note that $\mathfrak{j}_{0}$ (resp. $\left.\mathfrak{h}_{0}\right)$ is $G^{0} \times \mathbf{T}$-equivariant where $G^{0} \times \mathbf{T}$ acts on $Z_{\emptyset, D}$ by (a) (resp. by (b)). Since $A$ is equivariant for the action (a) for some $n$, it follows that ${ }^{p} H^{j}\left(\Phi_{u}^{\emptyset}(A)\right)$ is equivariant for the action (b). Hence any simple subquotient of ${ }^{p} H^{j}\left(\Phi_{u}^{\emptyset}(A)\right)$ is a character sheaf. This proves (c).

Let $K, K^{\prime} \subset \mathbf{I}$ and let $u \in{ }^{K} \mathbf{W}^{K^{\prime}}$. Let $H=K \cap \operatorname{Ad}(u) K^{\prime}, H^{\prime}=K^{\prime} \cap$ $\operatorname{Ad}\left(u^{-1}\right) K=\operatorname{Ad}\left(u^{-1}\right) H$. Let $\Phi_{u}: \mathcal{D}\left(Z_{H, D}\right) \rightarrow \mathcal{D}\left(Z_{H^{\prime}, D}\right)$ be as in 37.1. We show that

(d) $\Phi_{u} \mathfrak{f}_{\emptyset, H}(A)=\mathfrak{f}_{\emptyset, H^{\prime}} \Phi_{u}^{\emptyset}(A)$ for any $A \in \mathcal{D}\left(Z_{\emptyset, D}\right)$.

We have a diagram

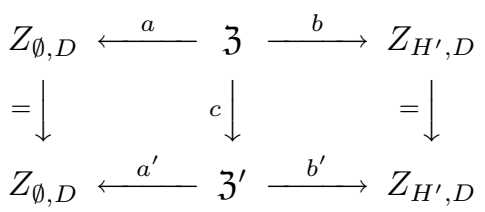

where

$$
\begin{gathered}
\mathfrak{Z}=\left\{\left(B, P, P^{\prime}, g\left(U_{P} \cap U_{P^{\prime}}\right) ; B \in \mathcal{B}, P \in \mathcal{P}_{H}, P^{\prime}\right.\right. \\
\left.\quad \in \mathcal{P}_{H^{\prime}}, g \in D, B \subset P, \operatorname{pos}\left(P, P^{\prime}\right)=u\right\}, \\
\mathfrak{Z}^{\prime}=\left\{\left(B, B^{\prime}, P^{\prime}, g\left(U_{B} \cap U_{B^{\prime}}\right), g^{\prime} U_{P^{\prime}}\right) ; B, B^{\prime} \in \mathcal{B}, P^{\prime}\right. \\
\left.\quad \in \mathcal{P}_{H^{\prime}}, g \in D ; g^{\prime} \in D, g U_{B^{\prime}}=g^{\prime} U_{B^{\prime}}, B^{\prime} \subset P^{\prime}, \operatorname{pos}\left(B, B^{\prime}\right)=u\right\},
\end{gathered}
$$

$a$ is $\left(B, P, P^{\prime}, g\left(U_{P} \cap U_{P^{\prime}}\right) \mapsto\left(B, g U_{B}\right)\right.$,

$b$ is $\left(B, P, P^{\prime}, g\left(U_{P} \cap U_{P^{\prime}}\right) \mapsto\left(P^{\prime}, g U_{P^{\prime}}\right)\right.$,

$a^{\prime}$ is $\left(B, B^{\prime}, P^{\prime}, g\left(U_{B} \cap U_{B^{\prime}}\right), g^{\prime} U_{P^{\prime}}\right) \mapsto\left(B, g U_{B}\right)$,

$b^{\prime}$ is $\left(B, B^{\prime}, P^{\prime}, g\left(U_{B} \cap U_{B^{\prime}}\right), g^{\prime} U_{P^{\prime}}\right) \mapsto\left(P^{\prime}, g U_{P^{\prime}}\right)$,

$c$ is $\left(B, P, P^{\prime}, g\left(U_{P} \cap U_{P^{\prime}}\right) \mapsto\left(\left(B, B^{\prime}, P^{\prime}, g\left(U_{B} \cap U_{B^{\prime}}\right), g U_{P^{\prime}}\right)\right.\right.$ with $B^{\prime}=$ $\left(B \cap P^{\prime}\right) U_{P^{\prime}}$.

From the definition and the change of base theorem we see that $\Phi_{u} \mathfrak{f}_{\emptyset, H}(A)=b_{!} a^{*} A$, $\mathfrak{f}_{\emptyset, H^{\prime}} \Phi_{u}^{\emptyset}(A)=b_{!}^{\prime} a^{\prime *} A$. Since $c$ is an isomorphism, the diagram above shows that $b_{!} a^{*} A=b_{!}^{\prime} a^{\prime *} A$. This proves (d).

We show that

(e) $\Phi_{u}$ restricts to a functor $\mathcal{D}^{c s}\left(Z_{H, D}\right) \rightarrow \mathcal{D}^{c s}\left(Z_{H^{\prime}, D}\right)$.

It is enough to show that if $A$ is a character sheaf on $Z_{H, D}$, then $\Phi_{u}(A) \in$ $\mathcal{D}^{c s}\left(Z_{H^{\prime}, D}\right)$. We can find a character sheaf $A^{\prime}$ on $Z_{\emptyset, D}$ and $m \in \mathbf{Z}$ such that $A[m]$ is a direct summand of $\mathfrak{f}_{\emptyset, H} A^{\prime}$. Hence $\Phi_{u}(A)[n]$ is a direct summand of $\Phi_{u} \mathfrak{f}_{\emptyset, H} A^{\prime}$. By (d), $\Phi_{u}(A)[n]$ is a direct summand of $\mathfrak{f}_{\emptyset, H^{\prime}} \Phi_{u}^{\emptyset}\left(A^{\prime}\right)$. It is enough to show that $\mathfrak{f}_{\emptyset, H^{\prime}} \Phi_{u}^{\emptyset}\left(A^{\prime}\right) \in \mathcal{D}^{c s}\left(Z_{H^{\prime}, D}\right)$. By (c), we have $\Phi_{u}^{\emptyset}\left(A^{\prime}\right) \in \mathcal{D}^{c s}\left(Z_{\emptyset, D}\right)$. Since $\mathfrak{f}_{\emptyset, H^{\prime}}$ carries $\mathcal{D}^{c s}\left(Z_{\emptyset, D}\right)$ into $\mathcal{D}^{c s}\left(Z_{\emptyset, H^{\prime}}\right)$, we see that (e) holds.

In the remainder of this section we assume that we are in the setup of 36.8. From 37.2 we deduce that for $K, K^{\prime}, J \subset \mathbf{I}$ such that $K \subset J, K^{\prime} \subset J$ we have

$$
\mathfrak{e}_{K^{\prime}, J} \mathfrak{f}_{K, J}=\sum_{u \in{ }^{K} \mathbf{W}^{K^{\prime}} \cap \mathbf{W}_{J}} v^{2 m_{u}} \mathfrak{f}_{K^{\prime} \cap \operatorname{Ad}\left(u^{-1}\right) K, K^{\prime}} \Phi_{u} \mathfrak{e}_{K \cap \operatorname{Ad}(u) K^{\prime}, K}
$$


as $\mathcal{A}$-linear maps $\mathfrak{K}\left(Z_{K, D}\right) \rightarrow \mathfrak{K}\left(Z_{K^{\prime}, D}\right)$, where the $\mathcal{A}$-linear map

$$
\operatorname{gr}\left(\Phi_{u}\right): \mathfrak{K}\left(Z_{K \cap \operatorname{Ad}(u) K^{\prime}, D}\right) \rightarrow \mathfrak{K}\left(Z_{K^{\prime} \cap \operatorname{Ad}\left(u^{-1}\right) K, D}\right)
$$

(see (e) and 36.8) is denoted again by $\Phi_{u}$.

37.4. Let $K, K^{\prime} \subset J$ and let $x \in \mathfrak{K}\left(Z_{K, D}\right)^{K}, x^{\prime} \in \mathfrak{K}\left(Z_{K^{\prime}, D}\right)^{K^{\prime}}$. We show that

$$
\left(\mathfrak{f}_{K, J} x: \mathfrak{f}_{K^{\prime}, J} x^{\prime}\right)=\sum_{u \in{ }^{K} \mathbf{W}^{K^{\prime}} \cap \mathbf{W}_{J} ; K=\operatorname{Ad}(u) K^{\prime}} v^{2 m_{u}}\left(\Phi_{u} x: x^{\prime}\right) .
$$

Here $m_{u}, \Phi_{u}$ are as in 37.3 ; in our case, $\Phi_{u}: \mathfrak{K}\left(Z_{K, D}\right) \rightarrow \mathfrak{K}\left(Z_{K^{\prime}, D}\right)$. Using 37.3 we have

$$
\begin{aligned}
& \left(\mathfrak{f}_{K, J} x: \mathfrak{f}_{K^{\prime}, J} x^{\prime}\right)=\left(\mathfrak{e}_{K^{\prime}, J} \mathfrak{f}_{K, J} x: x^{\prime}\right) \\
& =\left(\sum_{u \in \mathbf{W}^{K^{\prime}} \cap \mathbf{W}_{J}} v^{2 m_{u}} \mathfrak{f}_{K^{\prime} \cap \operatorname{Ad}\left(u^{-1}\right) K, K^{\prime}} \Phi_{u} \mathfrak{e}_{K \cap \operatorname{Ad}(u) K^{\prime}, K} x: x^{\prime}\right) \\
& =\sum_{u \in{ }^{K} \mathbf{W}^{K^{\prime}} \cap \mathbf{W}_{J}} v^{2 m_{u}}\left(\Phi_{u} \mathfrak{e}_{K \cap \operatorname{Ad}(u) K^{\prime}, K} x: \mathfrak{e}_{K^{\prime} \cap \operatorname{Ad}\left(u^{-1}\right) K, K^{\prime}} x^{\prime}\right) \\
& =\sum_{u \in{ }^{K} \mathbf{W}^{K^{\prime}} \cap \mathbf{W}_{J} ; K \cap \operatorname{Ad}(u) K^{\prime}=K, K^{\prime} \cap \operatorname{Ad}\left(u^{-1}\right) K=K^{\prime}} v^{2 m_{u}}\left(\Phi_{u} x: x^{\prime}\right) .
\end{aligned}
$$

The condition that $K \cap \operatorname{Ad}(u) K^{\prime}=K, K^{\prime} \cap \operatorname{Ad}\left(u^{-1}\right) K=K^{\prime}$ is equivalent to $K \subset \operatorname{Ad}(u) K^{\prime}, K^{\prime} \subset \operatorname{Ad}\left(u^{-1}\right) K$, that is, to $K=\operatorname{Ad}(u) K^{\prime}$. This proves (a).

Consider the equivalence relation on the set of subsets of $J$ given by $K_{1} \sim K_{2}$ if $\operatorname{Ad}(u) K_{1}=K_{2}$ for some $u \in \mathbf{W}_{J}$. For any equivalence class $\mathfrak{o}$ under $\sim$ we set

$$
\underline{\mathfrak{K}}\left(Z_{J, D}\right)^{\mathfrak{o}}=\sum_{H \subset J ; H \in \mathfrak{o}} f_{H, J}\left(\underline{\mathfrak{K}}\left(Z_{H, D}\right)^{H}\right) \subset \underline{\mathfrak{K}}\left(Z_{J, D}\right) .
$$

Note that $\underline{\mathfrak{K}}\left(Z_{J, D}\right)^{\mathfrak{0}}$ is homogeneous.

Proposition 37.5. We have

$$
\underline{\mathfrak{K}}\left(Z_{J, D}\right)=\bigoplus_{\mathfrak{o}} \underline{\mathfrak{K}}\left(Z_{J, D}\right)^{\mathfrak{o}}
$$

where $\mathfrak{o}$ runs over the equivalence classes for $\sim$.

If $J=\emptyset$ we have $\underline{\mathfrak{K}}\left(Z_{J, D}\right)=\underline{\mathfrak{K}}\left(Z_{J, D}\right)^{J}$ and the result is obvious. We may assume that $J \neq \emptyset$ and that the result is true when $J$ is replaced by a strictly smaller subset. Using 36.13(d) and the induction hypothesis we have

$$
\underline{\mathfrak{K}}\left(Z_{J, D}\right)=\underline{\mathfrak{K}}\left(Z_{J, D}\right)^{J}+\sum_{L \varsubsetneqq J} \mathfrak{f}_{L, J} \sum_{J^{\prime} ; J^{\prime} \subset L} \mathfrak{f}_{J^{\prime}, L} \underline{\mathfrak{K}}\left(Z_{J^{\prime}, D}\right)^{J^{\prime}} \subset \sum_{J^{\prime} ; J^{\prime} \subset J} \mathfrak{f}_{J^{\prime}, J} \underline{\mathfrak{K}}\left(Z_{J^{\prime}, D}\right)^{J^{\prime}} .
$$

Thus, $\underline{\mathfrak{K}}\left(Z_{J, D}\right)=\sum_{\mathfrak{o}} \underline{\mathfrak{K}}\left(Z_{J, D}\right)^{\mathfrak{o}}$. Next we show that $\left(\underline{\mathfrak{K}}\left(Z_{J, D}\right)^{\mathfrak{o}}: \underline{\mathfrak{K}}\left(Z_{J, D}\right)^{\mathfrak{o}^{\prime}}\right)=0$ if $\mathfrak{o} \neq \mathfrak{o}^{\prime}$. It is enough to show that $\left(\mathfrak{f}_{H, J}\left(\mathfrak{K}\left(Z_{H, D}\right)^{H}\right): \mathfrak{f}_{H^{\prime}, J}\left(\mathfrak{K}\left(Z_{H^{\prime}, D}\right)^{H^{\prime}}\right)\right)=0$ if $H, H^{\prime} \subset J, H \nsim H^{\prime}$. This follows from 37.4(a). It remains to use the following (easily verified) statement: If $V$ is a finite dimensional vector space with a nonsingular symmetric bilinear form (:) and $V_{1}, V_{2}, \ldots, V_{k}$ are subspaces such that $\left(V_{i}: V_{j}\right)=0$ for $i \neq j$ and $\sum_{i} V_{i}=V$, then $V=\bigoplus_{i} V_{i}$. 


\section{Duality}

38.1. We fix a connected component $D$ of $G$ that generates $G$. In this section we study an involution of the set of isomorphism classes of character sheaves on $D$ called duality.

We write $\epsilon$ instead of $\epsilon_{D}: \mathbf{W} \rightarrow \mathbf{W}$ (see 26.2). For $J \subset \mathbf{I}$ such that $\epsilon(J)=J$ we set, as in 30.3 ,

$$
V_{J, D}=\left\{\left(P, g U_{P}\right) ; P \in \mathcal{P}_{J}, g U_{P} \in N_{D} P / U_{P}\right\} .
$$

This is the same as ${ }^{1} Z_{J, D}$; see 36.2. We also set $\mathbf{W}_{J}^{\epsilon}=\left\{w \in \mathbf{W}_{J} ; \epsilon(w)=w\right\}$ where $\mathbf{W}_{J}$ is as in 26.1. Let $J_{\epsilon}$ be the set of orbits of the restriction of $\epsilon$ to $J$.

For any $P_{0} \in \mathcal{P}_{J}$ we have a functor $A_{0} \mapsto A_{0}^{b}$ (see 30.3) from the category of $P_{0} / U_{P_{0}}$-equivariant perverse sheaves on the connected component $N_{D} P_{0} / U_{P_{0}}$ of $N_{G} P_{0} / U_{P_{0}}$ to the category of perverse sheaves on $V_{J, D}$.

Let $C S\left(V_{J, D}\right)$ be the full subcategory of the category of perverse sheaves on $V_{J, D}$ whose objects are isomorphic to objects of the form $A_{0}^{b}$ where $A_{0}$ is a direct sum of character sheaves on $N_{D} P_{0} / U_{P_{0}}$. Equivalently, $C S\left(V_{J, D}\right)$ is the category of perverse sheaves on $V_{J, D}$ that are direct sums of perverse sheaves in ${ }^{1} \hat{Z}_{J, D}$; see 36.2 . We write also $C S(D)$ instead of $C S\left(V_{\mathbf{I}, D}\right)$. Note that $A_{0} \mapsto A_{0}^{b}$ is an equivalence of categories $C S\left(N_{D} P_{0} / U_{P_{0}}\right) \stackrel{\sim}{\longrightarrow} C S\left(V_{J, D}\right)$.

For $J \subset J^{\prime} \subset \mathbf{I}$ such that $\epsilon(J)=J, \epsilon\left(J^{\prime}\right)=J^{\prime}$ we have functors $f_{J, J^{\prime}}: \mathcal{D}\left(V_{J, D}\right) \rightarrow$ $\mathcal{D}\left(V_{J^{\prime}, D}\right)$ and $e_{J, J^{\prime}}: \mathcal{D}\left(V_{J^{\prime}, D}\right) \rightarrow \mathcal{D}\left(V_{J, D}\right)$; see 30.4. From definitions, for $J \subset J^{\prime} \subset$ $J^{\prime \prime} \subset$ I such that $\epsilon(J)=J, \epsilon\left(J^{\prime}\right)=J^{\prime}, \epsilon\left(J^{\prime \prime}\right)=J^{\prime \prime}$, we have

$$
f_{J, J^{\prime \prime}}=f_{J^{\prime}, J^{\prime \prime}} f_{J, J^{\prime}}, \quad e_{J, J^{\prime \prime}}=e_{J, J^{\prime}} e_{J^{\prime}, J^{\prime \prime}}
$$

Clearly, $f_{J, J}=1, e_{J, J}=1$.

38.2. We show that

(a) for $J \subset J^{\prime}$ as above, e $e_{J, J^{\prime}}$ restricts to a functor $C S\left(V_{J^{\prime}, D}\right) \rightarrow C S\left(V_{J, D}\right)$ denoted again by $e_{J, J^{\prime}}$.

Let $P \in \mathcal{P}_{J}, P^{\prime} \in \mathcal{P}_{J^{\prime}}$ be such that $P \subset P^{\prime}$. Let $D_{0}=N_{D} P / U_{P}, D_{0}^{\prime}=N_{D} P^{\prime} / U_{P^{\prime}}$. Let $C_{0} \in C S\left(D_{0}^{\prime}\right)$ and let $C=C_{0}^{b}$ be the corresponding object of $C S\left(V_{J^{\prime}, D}\right)$. From 31.14 we see that $\operatorname{res}_{D_{0}^{\prime}}^{D_{0}}\left(C_{0}\right) \in C S\left(D_{0}\right)$ and from $30.4(\mathrm{~b})$ we see that $e_{J, J^{\prime}} C$ is the perverse sheaf $\left(\operatorname{res}_{D_{0}^{\prime}}^{D_{0}}\left(C_{0}\right)\right)^{b}$ on $V_{J, D}$ hence $e_{J, J^{\prime}} C \in C S\left(V_{J, D}\right)$. This proves (a).

38.3. We show that

(a) for $J \subset J^{\prime}$ as above, $f_{J, J^{\prime}}$ restricts to a functor $C S\left(V_{J, D}\right) \rightarrow C S\left(V_{J^{\prime}, D}\right)$ denoted again by $f_{J, J^{\prime}}$.

Let $P, P^{\prime}, D_{0}, D_{0}^{\prime}$ be as in 38.2. Let $A_{0} \in C S\left(D_{0}\right)$ and let $A=A_{0}^{b}$ be the corresponding object of $C S\left(V_{J, D}\right)$. By $30.4(\mathrm{a}), f_{J, J^{\prime}} A=A_{0}^{\prime \text { b }}$ where $A_{0}^{\prime}=\operatorname{ind}_{D_{0}}^{D_{0}^{\prime}} A_{0}$ is a direct sum of simple admissible perverse sheaves on $D_{0}^{\prime}$. It remains to show that $A_{0}^{\prime} \in C S\left(D_{0}^{\prime}\right)$. To do this we may assume that $J^{\prime}=\mathbf{I}$, hence $P^{\prime}=G^{0}, D_{0}^{\prime}=D$. Let $\alpha=\operatorname{dim} U_{P}$. Let $L$ be a Levi of $P$. We can identify naturally $N_{G} P / U_{P}$ with $H=N_{G} P \cap N_{G} L$, a reductive group with $H^{0}=L$. Then $D_{0}$ becomes $H \cap D$. We identify the canonical torus of $L$ with the canonical torus $\mathbf{T}$ of $G^{0}$, and the Weyl group of $L$ with the subgroup $\mathbf{W}_{J}$ of $\mathbf{W}$ as in 29.1. Let $\mathcal{L} \in \mathfrak{s}$. Let $\mathbf{s}=\left(s_{1}, s_{2}, \ldots, s_{r}\right)$ be a sequence in $J \cup\{1\}$ such that $s_{1} s_{2} \ldots s_{r} \underline{D} \in \mathbf{W}_{\mathcal{L}}^{\bullet}$ (see 28.3). Let $\bar{Z}_{\emptyset, \mathbf{I}, D}^{\mathbf{s}}, \bar{K}_{D}^{\mathbf{s}, \mathcal{L}} \in \mathcal{D}(D)$ be as in $28.12, \overline{\mathcal{L}}$ as in 28.9 , and let $\bar{Z}_{\emptyset, J, D_{0}}^{\mathbf{s}}, \bar{K}_{D_{0}}^{\mathbf{s}, \mathcal{L}} \in \mathcal{D}\left(D_{0}\right)$, 
$\overline{\mathcal{L}}_{0}$, be the analogous objects defined in terms of $H, D_{0}$ instead of $G, D$. Consider the commutative diagram

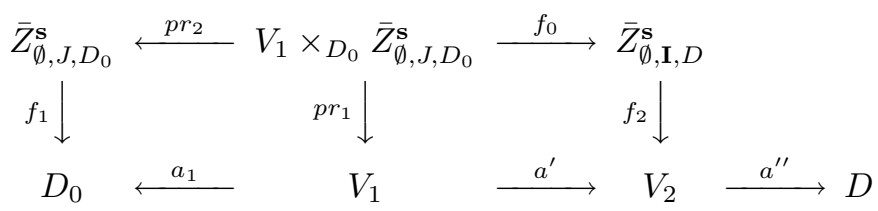

where

$V_{1}=\left\{(g, x) \in D \times G^{0} ; x^{-1} g x \in N_{D} P\right\}$,

$V_{2}=\left\{(g, x P) \in D \times G^{0} / P ; x^{-1} g x \in N_{D} P\right\}$,

$a_{1}(g, x)=g^{\prime}$ where $g_{0} \in D_{0}$ is such that $x^{-1} g x \in g_{0} U_{P}$,

$a^{\prime}(g, x)=(g, x P), a^{\prime \prime}(g, x P)=g, f_{1}\left(\beta_{0}, \beta_{1}, \ldots, \beta_{r}, g_{0}\right)=g_{0}$,

$f_{2}\left(B_{0}, B_{1}, \ldots, B_{r}, g\right)=\left(g, x_{0} P\right)$ where $x_{0} \in G^{0}$ is such that $x_{0}^{-1} B_{0} x_{0} \subset P$,

$f_{0}\left((g, x),\left(\beta_{0}, \beta_{1}, \ldots, \beta_{r}, g_{0}\right)\right)=\left(x \beta_{0} U_{P} x^{-1}, x \beta_{1} U_{P} x^{-1}, \ldots, x \beta_{r} U_{P} x^{-1}, g\right)$.

Both squares in the diagram are cartesian and the maps $a^{\prime}, f_{0}$ (resp. $a_{1}, p r_{2}$ ) are smooth with connected fibres of $\operatorname{dimension} \operatorname{dim} G-\alpha$ (resp. $\operatorname{dim} G+\alpha)$. From definitions we have $p r_{2}^{*} \overline{\mathcal{L}}^{\prime}=f_{0}^{*} \overline{\mathcal{L}}$. Hence

$$
a_{1}^{*}\left(\bar{K}_{D_{0}}^{\mathbf{s}, \mathcal{L}}\right)=a_{1}^{*} f_{1 !} \overline{\mathcal{L}}_{0}=p r_{1 !} p r_{2}^{*} \overline{\mathcal{L}}_{0}=p r_{1 !} f_{0}^{*} \overline{\mathcal{L}}=a^{\prime *} f_{2 !} \overline{\mathcal{L}} .
$$

We see that

$$
a_{1}^{\star}\left(\bar{K}_{D_{0}}^{\mathbf{s}, \mathcal{L}}\right)[-\operatorname{dim} G-\alpha]=a^{\prime \star} \tilde{K}[-\operatorname{dim} G+\alpha]
$$

where $\tilde{K}=f_{2 !} \overline{\mathcal{L}}$, that is, $a_{1}^{\star}\left(\bar{K}_{D_{0}}^{\mathbf{s}, \mathcal{L}}\right)=a^{\prime \star} \tilde{K}[2 \alpha]$. Hence

$$
\begin{aligned}
& a_{1}^{\star}\left({ }^{p} H^{i}\left(\bar{K}_{D_{0}}^{\mathbf{s}, \mathcal{L}}\right)\right)={ }^{p} H^{i}\left(a_{1}^{\star}\left(\bar{K}_{D_{0}}^{\mathbf{s}, \mathcal{L}}\right)\right)={ }^{p} H^{i}\left(a^{\prime \star} \tilde{K}[2 \alpha]\right)=a^{\prime \star}\left({ }^{p} H^{i}(\tilde{K}[2 \alpha])\right) \\
& =a^{\prime \star}\left({ }^{p} H^{i+2 \alpha} \tilde{K}\right) .
\end{aligned}
$$

From this and definition (27.1) we see that

$$
\operatorname{ind}_{D_{0}}^{D}\left({ }^{p} H^{i}\left(\bar{K}_{D_{0}}^{\mathbf{s}, \mathcal{L}}\right)\right)=a_{!}^{\prime \prime}\left({ }^{p} H^{i+2 \alpha} \tilde{K}\right) .
$$

We have

$$
\bigoplus_{i} \operatorname{ind}_{D_{0}}^{D}\left({ }^{p} H^{i}\left(\bar{K}_{D_{0}}^{\mathbf{s}, \mathcal{L}}\right)\right)[-i]=\bigoplus_{i}^{p} H^{i+2 \alpha}\left(\bar{K}_{D}^{\mathbf{s}, \mathcal{L}}\right)[-i] \in \mathcal{D}(D) .
$$

Indeed, the left-hand side is

$$
\begin{aligned}
\bigoplus_{i} & \left.a_{!}^{\prime \prime}\left({ }^{p} H^{i+2 \alpha} \tilde{K}\right)[-i]=a_{!}^{\prime \prime}(\tilde{K}[2 \alpha])=a_{!}^{\prime \prime} f_{2 !} \overline{\mathcal{L}}[2 \alpha]=\bar{K}_{D}^{\mathbf{s}, \mathcal{L}}\right)[2 \alpha] \\
& =\bigoplus_{i}{ }^{p} H^{i+2 \alpha}\left(\bar{K}_{D}^{\mathbf{s}, \mathcal{L}}\right)[-i] \in \mathcal{D}(D),
\end{aligned}
$$

where we use that $\tilde{K}$ and $\bar{K}_{D}^{\text {s, } \mathcal{L}}$ are semisimple complexes (a consequence of the decomposition theorem [BBD] $)$. Since ${ }^{p} H^{i}\left(\bar{K}_{D_{0}}^{\mathbf{s}, \mathcal{L}}\right)$ is a direct sum of character sheaves on $D_{0}$, we see, using $30.6(\mathrm{a})$, that $\operatorname{ind}_{D_{0}}^{D}\left({ }^{p} H^{i}\left(\bar{K}_{D_{0}}^{\mathbf{s}, \mathcal{L}}\right)\right)$ is a perverse sheaf on $D$. Taking ${ }^{p} H^{i}$ for both sides of (b) we therefore find

$$
\operatorname{ind}_{D_{0}}^{D}\left({ }^{p} H^{i}\left(\bar{K}_{D_{0}}^{\mathbf{s}, \mathcal{L}}\right)\right)={ }^{p} H^{i+2 \alpha}\left(\bar{K}_{D}^{\mathbf{s}, \mathcal{L}}\right)
$$

for any $i \in \mathbf{Z}$. To prove (a) it is enough to verify the following statement.

(d) If $A_{1} \in \hat{D}_{0}^{\mathcal{L}}$, then $\operatorname{ind}_{D_{0}}^{D}\left(A_{1}\right)$ is a direct sum of character sheaves in $\hat{D}^{\mathcal{L}}$. 
We may assume that $A_{1}$ is a direct summand of ${ }^{p} H^{i}\left(\bar{K}_{D_{0}}^{\mathbf{s}, \mathcal{L}}\right)$. Then $\operatorname{ind}_{D_{0}}^{D}\left(A_{1}\right)$ is a direct summand of $\operatorname{ind}_{D_{0}}^{D}\left({ }^{p} H^{i}\left(\bar{K}_{D_{0}}^{\mathbf{s}, \mathcal{L}}\right)\right)$. From (c) we see that $\operatorname{ind}_{D_{0}}^{D}\left(A_{1}\right)$ is a direct summand of ${ }^{p} H^{i+2 \alpha}\left(\bar{K}_{D}^{\mathbf{s}, \mathcal{L}}\right)$ which is a direct sum of character sheaves in $\hat{D}^{\mathcal{L}}$. This proves (d) hence also (a).

38.4. For $J \subset J^{\prime} \subset \mathbf{I}$ such that $\epsilon(J)=J, \epsilon\left(J^{\prime}\right)=J^{\prime}$ we have functors $\tilde{f}_{J, J^{\prime}}$ : $\mathcal{D}\left(V_{J, D}\right) \rightarrow \mathcal{D}\left(V_{J^{\prime}, D}\right), \tilde{e}_{J, J^{\prime}}: \mathcal{D}\left(V_{J^{\prime}, D}\right) \rightarrow \mathcal{D}\left(V_{J, D}\right)$; see 30.4.

Let $K, K^{\prime}, J \subset \mathbf{I}$ be such that $K \subset J, K^{\prime} \subset J, \epsilon(J)=J, \epsilon(K)=K, \epsilon\left(K^{\prime}\right)=K^{\prime}$. For any $u \in{ }^{K} \mathbf{W}^{K^{\prime}} \in \mathbf{W}_{J}^{\epsilon}$ let

$$
\begin{aligned}
& \Xi_{u}=\left\{\left(X, Y, g\left(U_{X} \cap U_{Y}\right)\right) ; X \in \mathcal{P}_{K \cap \operatorname{Ad}(u) K^{\prime}}, Y \in \mathcal{P}_{K^{\prime} \cap \operatorname{Ad}\left(u^{-1}\right) K},\right. \\
&\left.g\left(U_{X} \cap U_{Y}\right) \in\left(N_{D} X \cap N_{D} Y\right) /\left(U_{X} \cap U_{Y}\right), \operatorname{pos}(X, Y)=u\right\} .
\end{aligned}
$$

We have a diagram

$$
V_{K \cap \operatorname{Ad}(u) K^{\prime}, D} \stackrel{j}{\leftarrow} \Xi_{u} \stackrel{h}{\rightarrow} V_{K^{\prime} \cap \operatorname{Ad}\left(u^{-1}\right) K, D}
$$

where $j\left(X, Y, g\left(U_{X} \cap U_{Y}\right)\right)=\left(X, g U_{X}\right), h\left(X, Y, g\left(U_{X} \cap U_{Y}\right)\right)=\left(Y, g U_{Y}\right)$. Set $\Psi_{u}=$ $h_{!} j^{*}: \mathcal{D}\left(V_{K \cap \operatorname{Ad}(u) K^{\prime}, D}\right) \rightarrow \mathcal{D}\left(V_{K^{\prime} \cap \operatorname{Ad}\left(u^{-1}\right) K, D}\right)$.

Lemma 38.5. Let $A^{\prime} \in \mathcal{D}\left(V_{K, D}\right)$. We set $\tilde{\mathfrak{C}}=\tilde{e}_{K^{\prime}, J} \tilde{f}_{K, J} A^{\prime} \in \mathcal{D}\left(V_{K^{\prime}, D}\right)$. For any $u \in{ }^{K} \mathbf{W}^{K^{\prime}} \cap \mathbf{W}_{J}^{\epsilon}$ we set $\tilde{\mathfrak{C}}_{u}=\tilde{f}_{K^{\prime} \cap \operatorname{Ad}\left(u^{-1}\right) K, K^{\prime}} \Psi_{u} \tilde{e}_{K \cap \operatorname{Ad}(u) K^{\prime}, K} A^{\prime} \in \mathcal{D}\left(V_{K^{\prime}, D}\right)$ and $m_{u}=\operatorname{dim}\left(U_{P} \cap U_{R}\right) / U_{Q}$ where $P \in \mathcal{P}_{K}, R \in \mathcal{P}_{K^{\prime}}, \operatorname{pos}(P, R)=u$ and $Q=Q_{J, P}=$ $Q_{J, R} \in \mathcal{P}_{J}$ (notation of 36.4). We have

$$
\tilde{\mathfrak{C}} \approx\left\{\tilde{\mathfrak{C}}_{u}\left[\left[-m_{u}\right]\right] ; u \in{ }^{K} \mathbf{W}^{K^{\prime}} \cap \mathbf{W}_{J}^{\epsilon}\right\}
$$

where $\approx$ is as in 32.15.

The proof is very similar to that of Proposition 37.2. We have a commutative diagram with a cartesian square

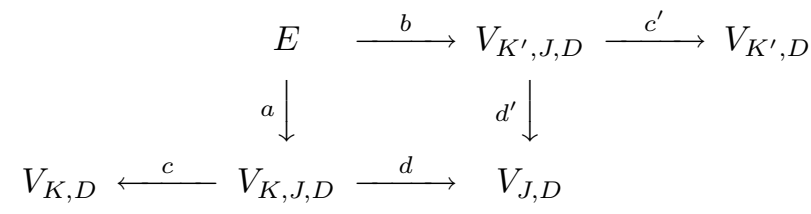

Here

$$
\begin{aligned}
& E=\left\{\left(P, R, g U_{Q}\right) ; P \in \mathcal{P}_{K}, R \in \mathcal{P}_{K^{\prime}}, g U_{Q}\right. \\
& \left.\quad \in\left(N_{D} P \cap N_{D} R\right) / U_{Q}, Q=Q_{J, P}=Q_{J, R}\right\}, \\
& c\left(P, g U_{Q}\right)=\left(P, g U_{P}\right), d\left(P, g U_{Q}\right)=\left(Q, g U_{Q}\right) \text { with } Q=Q_{J, P}, \\
& c^{\prime}\left(R, g U_{Q}\right)=\left(R, g U_{R}\right), d^{\prime}\left(R, g U_{Q}\right)=\left(Q, g U_{Q}\right), \text { with } Q=Q_{J, R}, \\
& a\left(P, R, g U_{Q}\right)=\left(P, g U_{Q}\right), b\left(P, R, g U_{Q}\right)=\left(R, g U_{Q}\right) .
\end{aligned}
$$

We have

$$
\tilde{\mathfrak{C}}=c_{!}^{\prime} d^{\prime *} d_{!} c^{*} A^{\prime}=c_{!}^{\prime} b_{!} a^{*} c^{*} A^{\prime}=\left(c^{\prime} b\right)_{!}(c a)^{*} A^{\prime}=q_{!} p^{*} A^{\prime}
$$

where $q=c^{\prime} b: E \rightarrow V_{K^{\prime}, D}, p=c a: E \rightarrow V_{K, D}$ are given by $q\left(P, R, g U_{Q}\right)=$ $\left(R, g U_{R}\right), p\left(P, R, g U_{Q}\right)=\left(P, g U_{P}\right)$. We have a partition

$$
E=\bigsqcup_{u \in \mathbf{W}^{K} \mathbf{W}^{\prime} \cap \mathbf{W}_{J}^{\epsilon}} E_{u}
$$


where $E_{u}=\left\{\left(P, R, g U_{Q}\right) \in E ; \operatorname{pos}(P, R)=u\right\}$ is locally closed in $E$. Let $p_{u}=$ $\left.p\right|_{E_{u}}: E_{u} \rightarrow V_{K, D}, q_{u}=\left.q\right|_{E_{u}}: E_{u} \rightarrow V_{K^{\prime}, D}$. By 32.15 , we have

$$
q ! p^{*} A^{\prime} \approx\left\{q_{u} ! p_{u}^{*} A^{\prime} ; u \in{ }^{K} \mathbf{W}^{K^{\prime}} \cap \mathbf{W}_{J}^{\epsilon}\right\}
$$

It remains to show that, for $u$ as above, we have $q_{u} p_{u}^{*} A^{\prime}=\tilde{\mathfrak{C}}_{u}\left[\left[-m_{u}\right]\right]$. We have a commutative diagram

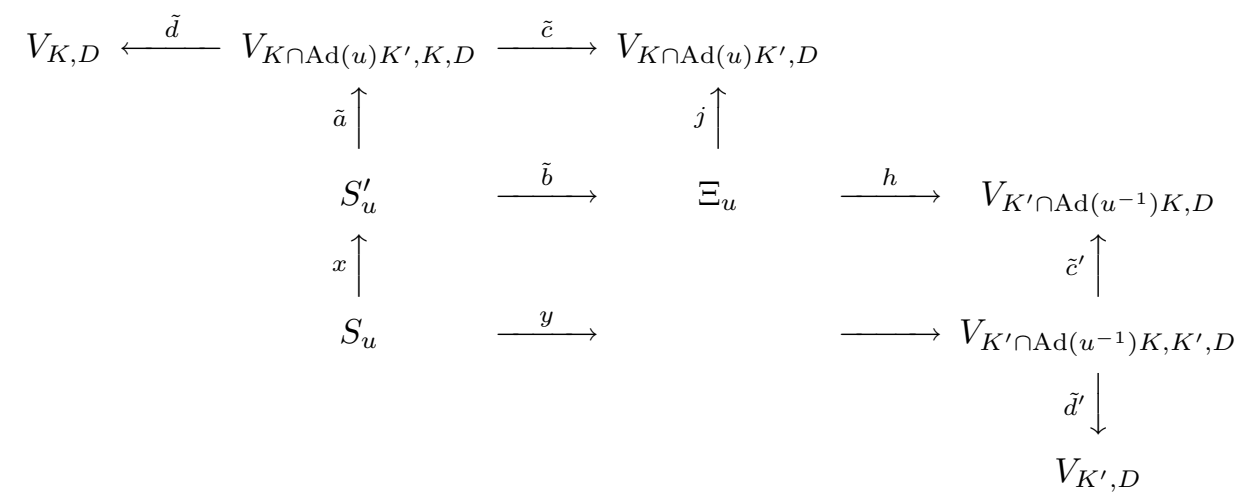

Here

$\tilde{c}\left(X, g U_{P}\right)=\left(X, g U_{X}\right), \tilde{d}\left(X, g U_{P}\right)=\left(P, g U_{P}\right)$,

$\tilde{c}^{\prime}\left(Y, g U_{R}\right)=\left(Y, g U_{Y}\right), \tilde{d}^{\prime}\left(Y, g U_{R}\right)=\left(R, g U_{R}\right)$,

$S_{u}^{\prime}, \tilde{a}, \tilde{b}$ are defined so that the square $(\tilde{a}, \tilde{b}, \tilde{c}, j)$ is cartesian;

$S_{u}, x, y$ are defined so that the square $\left(x, y, h \tilde{b}, \tilde{c}^{\prime}\right)$ is cartesian.

Then

$$
\begin{aligned}
& S_{u}=\left\{\left(X, Y, g\left(U_{X} \cap U_{Y}\right), g^{\prime} U_{P}, g^{\prime \prime} U_{R}\right) ; X \in \mathcal{P}_{K \cap \operatorname{Ad}(u) K^{\prime}}, Y \in \mathcal{P}_{K^{\prime} \cap \operatorname{Ad}\left(u^{-1}\right) K},\right. \\
& g\left(U_{X} \cap U_{Y}\right) \in\left(N_{D} X \cap N_{D} Y\right) /\left(U_{X} \cap U_{Y}\right), g^{\prime} U_{P} \in N_{D} X / U_{P}, \\
& g^{\prime \prime} U_{R} \in N_{D} Y / U_{R}, \operatorname{pos}(X, Y)=u, P=Q_{K, X}, R=Q_{K^{\prime}, Y} \\
& \left.g^{\prime} U_{X}=g U_{X}, g^{\prime \prime} U_{Y}=g U_{Y}\right\} .
\end{aligned}
$$

Set $r=\tilde{d}^{\prime} y: S_{u} \rightarrow V_{K^{\prime}, D}, s: \tilde{d} \tilde{a} x: S_{u} \rightarrow V_{K, D}$. Then

$$
\begin{aligned}
& r\left(X, Y, g\left(U_{X} \cap U_{Y}\right), g^{\prime} U_{P}, g^{\prime \prime} U_{R}\right)=\left(P, g^{\prime} U_{P}\right), \\
& s\left(X, Y, g\left(U_{X} \cap U_{Y}\right), g^{\prime} U_{P}, g^{\prime \prime} U_{R}\right)=\left(R, g^{\prime \prime} U_{R}\right) .
\end{aligned}
$$

We have

$$
\begin{aligned}
& \tilde{\mathfrak{C}}_{u}=\tilde{d}_{!}^{\prime} \tilde{c}^{\prime *} h_{!} j^{*} \tilde{c}_{!} \tilde{d}^{*} A^{\prime}=\tilde{d}_{!}^{\prime} \tilde{c}^{* *} h_{!} \tilde{b}_{!} \tilde{a}^{*} \tilde{d}^{*} A^{\prime}=\tilde{d}_{!}^{\prime} \tilde{c}^{*}(h \tilde{b}) !(\tilde{d} \tilde{a})^{*} A^{\prime} \\
& =\tilde{d}_{!}^{\prime} y ! x^{*}(\tilde{d} \tilde{a})^{*} A^{\prime}=\left(\tilde{d}^{\prime} y\right)_{!}(\tilde{d} \tilde{a} x)^{*} A^{\prime}=r ! s^{*} A^{\prime}
\end{aligned}
$$

We show that $q_{u} ! p_{u}^{*} A^{\prime}=r ! s^{*} A^{\prime}\left[\left[-m_{u}\right]\right]$. We have a commutative diagram

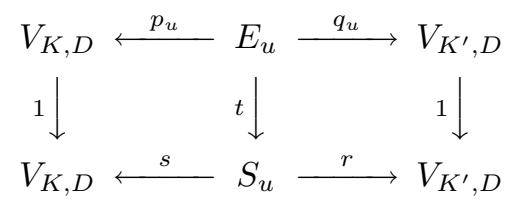

where $t\left(P, R, g U_{Q}\right)=\left(P^{R}, R^{P}, g\left(U_{P^{R}} \cap U_{R^{P}}\right), g U_{P}, g U_{R}\right)$ is well defined since $U_{Q} \subset$ $U_{P}, U_{Q} \subset U_{R}, U_{Q} \subset U_{P^{R}}, U_{Q} \subset U_{R^{P}}$. We continue the proof assuming that

(a) $t$ is an affine space bundle with fibres of dimension $m_{u}$. 
For any $\tilde{A} \in \mathcal{D}\left(S_{u}\right)$ we have $t_{!} t^{*}(\tilde{A})=\tilde{A}\left[\left[-m_{u}\right]\right]$. Hence

$$
r_{!} s^{*} A^{\prime}\left[\left[-m_{u}\right]\right]=r_{!} t_{!} t^{*} s^{*} A^{\prime}=(r t)_{!}(s t)^{*} A^{\prime}=q_{u} ! p_{u}^{*} A^{\prime},
$$

as required.

We prove (a). Consider the commutative diagram

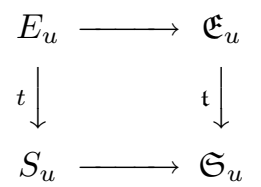

where $\mathfrak{E}_{u} \stackrel{\mathfrak{t}}{\rightarrow} \mathfrak{S}_{u}$ is as in the proof of 37.2 and the horizontal maps are the obvious imbeddings. Clearly, this diagram is cartesian. Hence (a) is a consequence of the analogous statement $37.2\left(\right.$ a) for $\mathfrak{E}_{u} \stackrel{\mathfrak{t}}{\rightarrow} \mathfrak{S}_{u}$. This completes the proof.

38.6. In the setup of 38.4 , let $u \in{ }^{K} \mathbf{W}^{K^{\prime}} \cap \mathbf{W}_{J}^{\epsilon}$. Let $\delta=\operatorname{dim}\left(U_{X_{u}} /\left(U_{X_{u}} \cap U_{Y_{u}}\right)\right)=$ $\operatorname{dim}\left(U_{Y_{u}} /\left(U_{X_{u}} \cap U_{Y_{u}}\right)\right)$ where $\left(X_{u}, Y_{u}, g\left(U_{X_{u}} \cap U_{Y_{u}}\right)\right) \in \Xi_{u}$. Let $\alpha_{u}=\operatorname{dim} U_{X_{u}}=$ $\operatorname{dim} U_{Y_{u}}, \alpha=\operatorname{dim} U_{P}, \alpha^{\prime}=\operatorname{dim} U_{R}$ where $P \in \mathcal{P}_{K}, R \in \mathcal{P}_{K^{\prime}}$. We show that

(a) $h$ and $j$ in 38.4 are affine space bundles with fibres of dimension $\delta$.

It is enough to prove the statements on $j$ (the statement on $h$ is entirely similar). We show that $j$ is surjective. Let $X, g$ be such that $\left(X, g U_{X}\right) \in V_{K \cap \operatorname{Ad}(u) K^{\prime}, D}$. We must show that there exist $Y \in \mathcal{P}_{K^{\prime} \cap \operatorname{Ad}\left(u^{-1}\right) K}, g^{\prime} \in N_{D} X \cap N_{D} Y$ such that $\operatorname{pos}(X, Y)=u$ and $g^{\prime} U_{X}=g U_{X}$. Setting $g^{-1} g^{\prime}=v$, it is enough to show that for any $Y \in \mathcal{P}_{K^{\prime} \cap \operatorname{Ad}\left(u^{-1}\right) K}$ with $\operatorname{pos}(X, Y)=u$ there exists $v \in U_{X}$ such that $g v \in N_{G} Y$. Now $X, Y$ contain a common Levi $M$. Since $g \in N_{G} X$, we can find $v \in U_{X}$ such that $g^{\prime}=g v \in N_{G} X \cap N_{G} M$. There is a unique parabolic $Y^{\prime}$ of the same type as $Y$ such that $Y^{\prime}$ has Levi $M$ and $\operatorname{pos}\left(X, Y^{\prime}\right)=u$. Then $\operatorname{pos}\left(g^{\prime} X g^{\prime-1}, g^{\prime} Y g^{\prime-1}\right)=u, g^{\prime} Y g^{\prime-1}$ has Levi $g^{\prime} M g^{\prime-1}=M$. By uniqueness, we have $Y^{\prime}=Y$. Thus, $g^{\prime} \in N_{G} Y$.

We show that the fibres of $j$ are affine spaces of dimension $\delta$. Let $\left(X, Y, g\left(U_{X} \cap\right.\right.$ $\left.\left.U_{Y}\right)\right) \in \Xi_{u}$. We must show that $F=\left\{\left(X, Y^{\prime}, g^{\prime}\left(U_{X} \cap U_{Y}^{\prime}\right)\right) \in \Xi_{u} ; g U_{X}=g^{\prime} U_{X}\right\}$ is an affine space. Fix $Y^{\prime} \in \mathcal{P}_{K^{\prime} \cap \operatorname{Ad}\left(u^{-1}\right) K}$ such that $\operatorname{pos}\left(X, Y^{\prime}\right)=u$. (The set of such $Y^{\prime}$ is a homogeneous space $U_{X} /\left(U_{X} \cap Y\right)$, hence is an affine space of dimension $\delta$.) It is enough to show that $\left\{g^{\prime}\left(U_{X} \cap U_{Y}^{\prime}\right) \in\left(N_{D} X \cap N_{D} Y\right) /\left(U_{X} \cap U_{Y}\right) ; g^{\prime} \in g U_{X}\right\}$ is a point. Now $g=g_{0} v_{0}$ where $g_{0} \in N_{D} X \cap N_{D} Y, v_{0} \in U_{X}$. It is enough to show that $\{v \in$ $\left.U_{X} ; v_{0} v \in N_{G} X \cap N_{G} Y\right\} /\left(U_{X} \cap U_{Y}\right)$ is a point or that $\left(U_{X} \cap N_{G} X \cap N_{G} Y\right) /\left(U_{X} \cap U_{Y}\right)$ is a point or that $U_{X} \cap Y=U_{X} \cap U_{Y}$. This is clear.

38.7. Let $u \in{ }^{K} \mathbf{W}^{K^{\prime}} \cap \mathbf{W}_{J}^{\epsilon}$. We set $H=K \cap \operatorname{Ad}(u) K^{\prime}, H^{\prime}=K^{\prime} \cap \operatorname{Ad}\left(u^{-1}\right) K=$ $\operatorname{Ad}\left(u^{-1}\right) H$. Let $\Psi_{u}^{\prime}=\Psi_{u}[[\delta]]: \mathcal{D}\left(V_{H, D}\right) \rightarrow \mathcal{D}\left(V_{H^{\prime}, D}\right)$. We show that

(a) If $A \in C S\left(V_{H, D}\right)$, then $\Psi_{u}^{\prime}(A) \in C S\left(V_{H^{\prime}, D}\right)$.

We have a commutative diagram in which the upper squares are cartesian and the 
left and right vertical arrows are smooth with connected fibres:

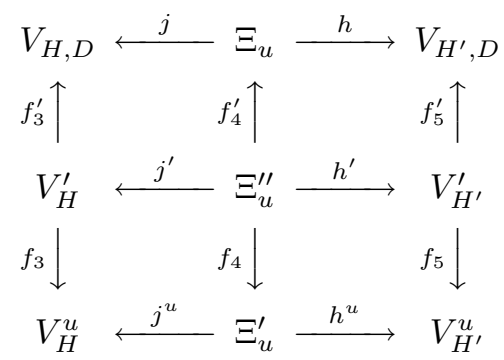

Here

$$
\begin{aligned}
& V_{H}^{u}=\left\{\left(X, g U_{X}\right) \in V_{H, D} ; X=X_{u}\right\}, V_{H^{\prime}}^{u}=\left\{\left(Y, g U_{Y}\right) \in V_{H^{\prime}, D} ; Y=Y_{u}\right\} \\
& \Xi_{u}^{\prime}=\left\{\left(X, Y, g\left(U_{X} \cap U_{Y}\right)\right) \in \Xi_{u} ; X=X_{u}, Y=Y_{u}\right\} \\
& V_{H}^{\prime}=G^{0} / U_{X_{u}} \times V_{H}^{u}, V_{H^{\prime}}^{\prime}=G^{0} / U_{Y_{u}} \times V_{H^{\prime}}^{u}, \\
& \Xi_{u}^{\prime \prime}=G^{0} /\left(U_{X_{u}} \cap U_{Y_{u}}\right) \times \Xi_{u}^{\prime}, \\
& j^{u}, h^{u} \text { are the restrictions of } j, h, \\
& j^{\prime}\left(x\left(U_{X_{u}} \cap U_{Y_{u}}\right),\left(X_{u}, Y_{u}, g\left(U_{X_{u}} \cap U_{Y_{u}}\right)\right)=\left(x U_{X_{u}},\left(X_{u}, g U_{X_{u}}\right)\right),\right. \\
& h^{\prime}\left(x\left(U_{X_{u}} \cap U_{Y_{u}}\right),\left(X_{u}, Y_{u}, g\left(U_{X_{u}} \cap U_{Y_{u}}\right)\right)=\left(x U_{Y_{u}},\left(Y_{u}, g U_{Y_{u}}\right)\right),\right. \\
& \left.f_{3}^{\prime}\left(x U_{X_{u}},\left(X_{u}, g U_{X_{u}}\right)\right)=\left(x X_{u} x^{-1}, x g x^{-1} U_{x X_{u} x^{-1}}\right)\right), \\
& f_{3}\left(x U_{X_{u}},\left(X_{u}, g U_{X_{u}}\right)\right)=\left(X_{u}, g U_{X_{u}}\right), \\
& f_{4}^{\prime}\left(x\left(U_{X_{u}} \cap U_{Y_{u}}\right),\left(X_{u}, Y_{u}, g\left(U_{X_{u}} \cap U_{Y_{u}}\right)\right)=\left(x X_{u} x^{-1}, x Y_{u} x^{-1}, x g x^{-1}\left(U_{x X_{u} x^{-1}} \cap\right.\right.\right. \\
& U_{\left.\left.x Y_{u} x^{-1}\right)\right)} \\
& f_{5}^{\prime}\left(x U_{Y_{u}},\left(Y_{u}, g U_{Y_{u}}\right)\right)=\left(x Y_{u} x^{-1}, x g x^{-1} U_{x Y_{u} x^{-1}}\right), \\
& f_{4}\left(x\left(U_{X_{u}} \cap U_{Y_{u}}\right),\left(X_{u}, Y_{u}, g\left(U_{X_{u}} \cap U_{Y_{u}}\right)\right)=\left(X_{u}, Y_{u}, g\left(U_{X_{u}} \cap U_{Y_{u}}\right)\right),\right. \\
& f_{5}\left(x Y_{u},\left(Y_{u}, g U_{Y_{u}}\right)\right)=\left(Y_{u}, g U_{Y_{u}}\right) .
\end{aligned}
$$

We may identify $V_{H}^{u}=N_{D} X_{u} / U_{X_{u}}, V_{H^{\prime}}^{u}=N_{D} Y_{u} / U_{Y_{u}}$ in an obvious way. We can find $C \in C S\left(N_{D} X_{u} / U_{X_{u}}\right)$ such that $f_{3}^{\prime \star} A=f_{3}^{\star} C$. Hence $f_{3}^{\prime *} A\left[\operatorname{dim} X_{u} / U_{X_{u}}\right]=$ $f_{3}^{*} C\left[\operatorname{dim} G / U_{X_{u}}\right]$ and $f_{3}^{\prime *} A=f_{3}^{*} A_{1}$ where $A_{1}=C\left[\alpha_{u}\right]$. Let $A_{2}=h_{!}^{u} j^{u *} A_{1}, A_{2}^{\prime}=$ $h_{!} j^{*} A$. Using 38.6(a) and the fact that $h^{u}$ is an isomorphism, we have

$$
\begin{aligned}
& f_{5}^{\prime *} A_{2}^{\prime}=f_{5}^{\prime *} h_{!} j^{*} A=h_{!}^{\prime} f_{4}^{\prime *} j^{*} A=h_{!}^{\prime} j^{\prime *} f_{3}^{\prime *} A=h_{!}^{\prime} j^{* *} f_{3}^{*} A_{1}=h_{!}^{\prime} f_{4}^{*} j^{u *} A_{1} \\
& =h_{!}^{\prime} f_{4}^{*} h^{u *} h_{!}^{u} j^{u *} A_{1}=h_{!}^{\prime} h^{\prime *} f_{5}^{*} h_{!}^{u} j^{u *} A_{1}=f_{5}^{*} h_{!}^{u} j^{u *} A_{1}[[-\delta]]=f_{5}^{*} A_{2}[[-\delta]] .
\end{aligned}
$$

Thus, $f_{5}^{*} A_{2}[[-\delta]]=f_{5}^{\prime *} A_{2}^{\prime}$. Hence

$$
f_{5}^{\star} A_{2}\left[-\operatorname{dim} G^{0} / U_{Y_{u}}\right][[-\delta]]=f_{5}^{\prime \star} A_{2}^{\prime}\left[-\operatorname{dim} Y_{u} / U_{Y_{u}}\right], f_{5}^{\star} A_{2}\left[-\alpha_{u}\right]=f_{5}^{\prime \star} A_{2}^{\prime}[[\delta]] .
$$

Since $h^{u}, j^{u}$ are isomorphisms we see that $A_{2}\left[-\alpha_{u}\right]$ is a perverse sheaf. Hence so is $f_{5}^{\star} A_{2}\left[-\alpha_{u}\right]$. Hence $f_{5}^{\prime \star} A_{2}^{\prime}[[\delta]]$ is perverse. Hence $A_{2}^{\prime}[[\delta]]$ is perverse and $h_{!} j^{*} A[[\delta]]$ is perverse. To show that $h_{!} j^{*} A[[\delta]]=A_{2}^{\prime}[[\delta]] \in C S\left(V_{H^{\prime}, D}\right)$, it is enough to show that $A_{2}\left[-\alpha_{u}\right] \in C S\left(N_{D} Y_{u} / U_{Y_{u}}\right)$ or that $h_{!}^{u} j{ }^{u *} C \in C S\left(N_{D} Y_{u} / U_{Y_{u}}\right)$.

Let $M$ be a common Levi subgroup of $X_{u}, Y_{u}$. Let $\tilde{M}=N_{G} X_{u} \cap N_{G} Y_{u} \cap N_{G} M$. Then $\tilde{M}$ is a reductive group with $\tilde{M}^{0}=M$ and $\tilde{M}^{1}=N_{D} X_{u} \cap N_{D} Y_{u} \cap N_{D} M$ is a connected component of $\tilde{M}$. Moreover, the obvious maps $\tilde{M}^{1} \rightarrow N_{D} X_{u} / U_{X_{u}}$, $\tilde{M}^{1} \rightarrow N_{D} Y_{u} / U_{Y_{u}}, \tilde{M}^{1} \rightarrow\left(N_{D} X_{u} \cap N_{D} Y_{u}\right) /\left(U_{X_{u}} \cap U_{Y_{u}}\right)$ are isomorphisms (see 1.25). Hence the bottom row $V_{H}^{u} \stackrel{j^{u}}{\longleftarrow} \Xi_{u}^{\prime} \stackrel{h^{u}}{\longrightarrow} V_{H^{\prime}}^{u}$ of the commutative diagram above may be identified with $\tilde{M}^{1} \leftarrow \tilde{M}^{1} \rightarrow \tilde{M}^{1}$ where both maps are the identity. Thus $h_{!}^{u} j^{u *} C \in C S\left(N_{D} Y_{u} / U_{Y_{u}}\right)$ follows immediately from $C \in C S\left(N_{D} X_{u} / U_{X_{u}}\right)$. This proves (a).

We now show that (b) for $A$ as above we have $f_{H^{\prime}, J} \Psi_{u}^{\prime}(A)=f_{H, J}(A)$. 
Let $Q \in \mathcal{P}_{J}$ be such that $X_{u} \subset Q \supset Y_{u}$. Let $M_{1}$ be the unique Levi of $Q$ such that $M \subset M_{1}$. Let $\tilde{M}_{1}=N_{G} Q \cap N_{G} M_{1}$. Then $\tilde{M}_{1}$ is a reductive group with $\tilde{M}_{1}^{0}=M_{1}$ and $\tilde{M}_{1}^{1}=N_{D} Q \cap N_{D} M_{1}$ is a connected component of $\tilde{M}_{1}$. Moreover, $X_{u} \cap \tilde{M}_{1}, Y_{u} \cap \tilde{M}_{1}$ are parabolic subgroups of $M_{1}$ with a common Levi, $M$. Let $C \in C S\left(N_{D} X_{u} / U_{X_{u}}\right)=C S\left(\tilde{M}^{1}\right)$ be as above. We may assume that $A$ is simple so that $C$ is also simple. Using the proof of (a) and that of 38.2 we see that it is enough to verify that $\operatorname{ind}_{\tilde{M}^{1}}^{\tilde{M}_{1}^{1}}(C)$ (defined in terms of the parabolic $X_{u} \cap \tilde{M}_{1}$ ) is isomorphic to $\operatorname{ind}_{\tilde{M}^{1}}^{\tilde{M}_{1}^{1}}(C)$ (defined in terms of the parabolic $Y_{u} \cap \tilde{M}_{1}$ ). Since $C$ is an admissible complex on $\tilde{M}^{1}$ (see 30.12), this follows from 27.2(d) which shows (for $G$ instead of $\left.\tilde{M}_{1}\right)$ that $\operatorname{ind}_{\tilde{M}_{1}^{1}}^{\tilde{M}_{1}^{1}}(C)$ can be defined without reference to a choice of parabolic. This proves (b).

Proposition 38.8. Let $K, K^{\prime}, J$ be as in 38.4. Let $A \in C S\left(V_{K, D}\right)$. We have

$$
e_{K^{\prime}, J} f_{K, J} A \cong \bigoplus_{u \in{ }^{K} \mathbf{W}^{K^{\prime}} \cap \mathbf{W}_{J}^{\epsilon}} f_{K^{\prime} \cap \operatorname{Ad}\left(u^{-1}\right) K, K^{\prime}} \Psi_{u}^{\prime} e_{K \cap \operatorname{Ad}(u) K^{\prime}, K} A
$$

in $C S\left(V_{K^{\prime}, D}\right)$.

We set $\mathfrak{C}=e_{K^{\prime}, J} f_{K, J} A \in \mathcal{D}\left(V_{K, D}\right)$. For any $u \in{ }^{K} \mathbf{W}^{K^{\prime}} \cap \mathbf{W}_{J}^{\epsilon}$ we set $\mathfrak{C}_{u}=$ $f_{K^{\prime} \cap \operatorname{Ad}\left(u^{-1}\right) K, K^{\prime}} \Psi_{u}^{\prime} e_{K \cap \operatorname{Ad}(u) K^{\prime}, K} A \in \mathcal{D}\left(V_{K^{\prime}, D}\right)$. Assume that we can show that

$$
\mathfrak{C} \approx\left\{\mathfrak{C}_{u} ; u \in{ }^{K} \mathbf{W}^{K^{\prime}} \cap \mathbf{W}_{J}^{\epsilon}\right\} .
$$

From the definition of $\approx$ (see 32.15$)$ it would then follow that

$$
\sum_{i}(-1)^{i}\left({ }^{p} H^{i}(\mathfrak{C})\right)=\sum_{u \in{ }^{K} \mathbf{W}^{K^{\prime}} \cap \mathbf{W}_{J}^{\epsilon}} \sum_{i}(-1)^{i}\left({ }^{p} H^{i}\left(\mathfrak{C}_{u}\right)\right)
$$

in the Grothendieck group of the category of perverse sheaves on $V_{K^{\prime}, D}$. From 38.2(a), 38.3(a), 38.7(a) we see that $\mathfrak{C}, \mathfrak{C}_{u} \in C S\left(V_{K^{\prime}, D}\right)$ (hence are perverse sheaves); hence the previous equality implies that $\mathfrak{C}=\sum_{u \in{ }^{K} \mathbf{W}^{K^{\prime}} \cap \mathbf{W}_{J}^{\epsilon}} \mathfrak{C}_{u}$ in the Grothendieck group of the category of perverse sheaves on $V_{K^{\prime}, D}$. Since $\mathfrak{C}, \mathfrak{C}_{u}$ are semisimple perverse sheaves (being in $C S\left(V_{K^{\prime}, D}\right)$ ) it follows that $\mathfrak{C} \cong \bigoplus_{u \in{ }^{K} \mathbf{W}^{K^{\prime}} \cap \mathbf{W}_{J}^{\epsilon}} \mathfrak{C}_{u}$, as desired.

Assume that $P \in \mathcal{P}_{K}$ contains $X_{u}$ and $R \in \mathcal{P}_{K^{\prime}}$ contains $Y_{u}$ so that $\operatorname{pos}(P, R)=$ $u$. $\left(X_{u}, Y_{u}\right.$ as in 38.6.) Let $Q \in \mathcal{P}_{J}$ be such that $P \subset Q \supset R$. Let $\beta=\operatorname{dim} U_{Q}$. We have

$\mathfrak{C}=\tilde{\mathfrak{C}}\left[\alpha+\alpha^{\prime}-2 \beta\right]\left(\alpha^{\prime}-\beta\right)$,

$\mathfrak{C}_{u}=\tilde{\mathfrak{C}}_{u}\left[\alpha_{u}-\alpha\right]\left(\alpha_{u}-\alpha\right)\left[\alpha_{u}-\alpha^{\prime}\right][2 \delta](\delta)$.

(Notation of 38.5.) Hence it is enough to show

$\tilde{\mathfrak{C}}\left[\alpha+\alpha^{\prime}-2 \beta\right]\left(\alpha^{\prime}-\beta\right) \approx\left\{\tilde{\mathfrak{C}}_{u}\left[2 \alpha_{u}-\alpha-\alpha^{\prime}+2 \delta\right]\left(\alpha_{u}-\alpha+\delta\right) ; u \in{ }^{K} \mathbf{W}^{K^{\prime}} \cap \mathbf{W}_{J}^{\epsilon}\right\}$

or that

$$
\tilde{\mathfrak{C}} \approx\left\{\tilde{\mathfrak{C}}_{u}\left[2 \alpha_{u}-2 \alpha-2 \alpha^{\prime}+2 \delta-2 \beta\right]\left(\alpha_{u}-\alpha-\alpha^{\prime}+\delta+\beta\right) ; u \in{ }^{K} \mathbf{W}^{K^{\prime}} \cap \mathbf{W}_{J}^{\epsilon}\right\} .
$$

By 38.5, it is enough to show that for any $u$ we have $\alpha_{u}-\alpha-\alpha^{\prime}+\delta+\beta=-m_{u}$ or that

$$
\operatorname{dim} U_{P}+\operatorname{dim} U_{R}-\operatorname{dim}\left(U_{P} \cap U_{R}\right)=\operatorname{dim} U_{X_{u}}+\operatorname{dim} U_{Y_{u}}-\operatorname{dim}\left(U_{X_{u}} \cap U_{Y_{u}}\right) .
$$


It is enough to show that

$$
\begin{aligned}
& \operatorname{dim} \operatorname{Lie} U_{P}+\operatorname{dim} \operatorname{Lie} U_{R}-\operatorname{dim}\left(\operatorname{Lie} U_{P} \cap \text { Lie } U_{R}\right) \\
& =\operatorname{dim} \operatorname{Lie} U_{X_{u}}+\operatorname{dim} \operatorname{Lie} U_{Y_{u}}-\operatorname{dim}\left(\operatorname{Lie} U_{X_{u}} \cap \operatorname{Lie} U_{Y_{u}}\right)
\end{aligned}
$$

or that $\operatorname{dim}\left(\right.$ Lie $U_{P}+$ Lie $\left.U_{R}\right)=\operatorname{dim}\left(\right.$ Lie $U_{X_{u}}+$ Lie $\left.U_{Y_{u}}\right)$. We have

Lie $U_{X_{u}}=$ Lie $U_{P}+\left(\right.$ Lie $P \cap$ Lie $\left.U_{R}\right)$, Lie $U_{Y_{u}}=$ Lie $U_{R}+\left(\right.$ Lie $R \cap$ Lie $\left.U_{P}\right)$, hence Lie $U_{X_{u}}+$ Lie $U_{Y_{u}} \subset$ Lie $U_{P}+$ Lie $U_{R}$. The opposite inclusion is clear since Lie $U_{P} \subset$ Lie $U_{X_{u}}$, Lie $U_{R} \subset$ Lie $U_{Y_{u}}$. Thus we have

$$
\text { Lie } U_{X_{u}}+\text { Lie } U_{Y_{u}}=\text { Lie } U_{P}+\text { Lie } U_{R} \text {. }
$$

This completes the proof.

38.9. For $J \subset \mathbf{I}$ such that $\epsilon(J)=J$ let $\mathcal{K}\left(V_{J, D}\right)$ be the Grothendieck group of $C S\left(V_{J, D}\right)$. Similarly let $\mathcal{K}(D)$ be the Grothendieck group of $C S(D)$. For $A, A^{\prime} \in$ $C S\left(V_{J, D}\right)$ we set $\left(A, A^{\prime}\right)=\operatorname{dim} \operatorname{Hom}\left(A, A^{\prime}\right)$. This induces a symmetric bilinear pairing $(,)_{J}: \mathcal{K}\left(V_{J, D}\right) \times \mathcal{K}\left(V_{J, D}\right) \rightarrow \mathbf{Z}$.

For $J \subset J^{\prime} \subset \mathbf{I}$ such that $\epsilon(J)=J, \epsilon\left(J^{\prime}\right)=J^{\prime}$, the functors $f_{J, J^{\prime}}: C S\left(V_{J, D}\right) \rightarrow$ $C S\left(V_{J^{\prime}, D}\right)$ and $e_{J, J^{\prime}}: C S\left(V_{J^{\prime}, D}\right) \rightarrow C S\left(V_{J, D}\right)$ are compatible with direct sums hence they induce homomorphisms $\mathcal{K}\left(V_{J, D}\right) \rightarrow \mathcal{K}\left(V_{J^{\prime}, D}\right), \mathcal{K}\left(V_{J^{\prime}, D}\right) \rightarrow \mathcal{K}\left(V_{J, D}\right)$ denoted again by $f_{J, J^{\prime}}, e_{J, J^{\prime}}$. From 30.5 we see that

$$
\left(e_{J, J^{\prime}} A^{\prime}, A\right)_{J}=\left(A^{\prime}, f_{J, J^{\prime}} A\right)_{J^{\prime}}
$$

for $A \in \mathcal{K}\left(V_{J, D}\right), A^{\prime} \in \mathcal{K}\left(V_{J, D}\right)$.

In the setup of 38.7 , for $u \in{ }^{K} \mathbf{W}^{K^{\prime}} \in \mathbf{W}_{J}^{\epsilon}$, the functor

$$
\Psi_{u}^{\prime}: C S\left(V_{K \cap \operatorname{Ad}(u) K^{\prime}, D}\right) \rightarrow C S\left(V_{K^{\prime} \cap \operatorname{Ad}\left(u^{-1}\right) K, D}\right)
$$

is compatible with direct sums hence induces a homomorphism

$$
\mathcal{K}\left(V_{K \cap \operatorname{Ad}(u) K^{\prime}, D}\right) \rightarrow \mathcal{K}\left(V_{K^{\prime} \cap \operatorname{Ad}\left(u^{-1}\right) K, D}\right.
$$

denoted again by $\Psi_{u}^{\prime}$. Below we shall need the following identity:

$$
\sum_{\substack{K^{\prime} ; K^{\prime} \subset J \\ \epsilon\left(K^{\prime}\right)=K}} \sum_{\substack{u \in \mathbf{W}^{K^{\prime}} \cap \mathbf{W}_{J}^{\epsilon} \\ K \cap \operatorname{Ad}(u) K^{\prime}=H}}(-1)^{\left|K_{\epsilon}^{\prime}\right|}=(-1)^{\left|H_{\epsilon}\right|}
$$

for any $H \subset K \subset J$ such that $\epsilon(H)=H, \epsilon(K)=K$. In the case where $J=\mathbf{I}, \epsilon=1$ this is proved in $[\mathrm{Cu}, 2.5]$; the general case can be reduced to this special case by replacing $\mathbf{W}$ by $\mathbf{W}_{J}^{\epsilon}$ which is itself a Weyl group with simple reflections in bijection with $J_{\epsilon}$.

38.10. For $J \subset \mathbf{I}$ such that $\epsilon(J)=J$ we define a homomorphism $\mathbf{d}_{J}: \mathcal{K}\left(V_{J, D}\right) \rightarrow$ $\mathcal{K}\left(V_{J, D}\right)$ by

$$
\mathbf{d}_{J} A=\sum_{K ; K \subset J, \epsilon(K)=K}(-1)^{\left|K_{\epsilon}\right|} f_{K, J} e_{K, J} A .
$$

Using 38.9(a) we see that for $A, A^{\prime} \in \mathcal{K}\left(V_{J, D}\right)$ we have

$$
\left(\mathbf{d}_{J}(A), A^{\prime}\right)_{J}=\left(A, \mathbf{d}_{J}\left(A^{\prime}\right)_{J} .\right.
$$

We show that, for $K \subset J \subset \mathbf{I}$ such that $\epsilon(K)=K, \epsilon(J)=J$ and $A \in \mathcal{K}\left(V_{K, D}\right)$, we have

$$
\mathbf{d}_{J} f_{K, J} A=f_{K, J} \mathbf{d}_{K} A .
$$


Using 38.8, 38.7(b), 38.9(b), 38.1(a) we have

$$
\begin{aligned}
& \mathbf{d}_{J} f_{K, J} A=\sum_{K^{\prime} ; K^{\prime} \subset J, \epsilon\left(K^{\prime}\right)=K^{\prime}}(-1)^{\left|K_{\epsilon}^{\prime}\right|} f_{K^{\prime}, J} e_{K^{\prime}, J} f_{K, J} A \\
& =\sum(-1)^{\left|K_{\epsilon}^{\prime}\right|} f_{K^{\prime}, J} f_{K^{\prime} \cap \operatorname{Ad}\left(u^{-1}\right) K, K^{\prime}} \Psi_{u}^{\prime} e_{K \cap \operatorname{Ad}(u) K^{\prime}, K} A \\
& =\sum(-1)^{\left|K_{\epsilon}^{\prime}\right|} f_{\operatorname{Ad}\left(u^{-1}\right) H, J} \Psi_{u}^{\prime} e_{H, K} A \\
& =\sum(-1)^{\left|K_{\epsilon}^{\prime}\right|} f_{H, J} e_{H, K} A \\
& =\sum(-1)^{\left|K_{\epsilon}^{\prime}\right|} f_{H, J} e_{H, K} A=\sum_{H ; H \subset K, \epsilon(H)=H}(-1)^{\left|H_{\epsilon}\right|} f_{H, J} e_{H, K} A \\
& =\sum_{H ; H \subset K, \epsilon(H)=H}(-1)^{\left|H_{\epsilon}\right|} f_{K, J} f_{H, K} e_{H, K} A=f_{K, J} \mathbf{d}_{K} A
\end{aligned}
$$

and (c) is proved.

We show that, for $J \subset \mathbf{I}$ such that $\epsilon(J)=J$ and $A \in \mathcal{K}\left(V_{J, D}\right)$, we have

$$
\mathbf{d}_{J} \mathbf{d}_{J} A=A \text {. }
$$

Using (c), 38.1(a), we have

$$
\begin{aligned}
& \mathbf{d}_{J} \mathbf{d}_{J} A=\sum_{K ; K \subset J, \epsilon(K)=K}(-1)^{\left|K_{\epsilon}\right|} \mathbf{d}_{J} f_{K, J} e_{K, J} A \\
& =\sum_{K ; K \subset J, \epsilon(K)=K}(-1)^{\left|K_{\epsilon}\right|} f_{K, J} \mathbf{d}_{K} e_{K, J} A \\
& =\sum_{K, K^{\prime} ; K^{\prime} \subset K \subset J, \epsilon(K)=K, \epsilon\left(K^{\prime}\right)=K^{\prime}}(-1)^{\left|K_{\epsilon}\right|}(-1)^{\left|K_{\epsilon}^{\prime}\right|} f_{K, J} f_{K^{\prime}, K} e_{K^{\prime}, K} e_{K, J} A \\
& =\sum_{K, K^{\prime} ; K^{\prime} \subset K \subset J, \epsilon(K)=K, \epsilon\left(K^{\prime}\right)=K^{\prime}}(-1)^{\left|K_{\epsilon}\right|}(-1)^{\left|K_{\epsilon}^{\prime}\right|} f_{K^{\prime}, J} e_{K^{\prime}, J} A \\
& =\sum_{K^{\prime} ; K^{\prime} \subset J, \epsilon\left(K^{\prime}\right)=K^{\prime}}(-1)^{\left|K_{\epsilon}^{\prime}\right|} \sum_{K^{\prime} ; K^{\prime} \subset J, \epsilon\left(K^{\prime}\right)=K^{\prime}}^{K ; K^{\prime} \subset K \subset J, \epsilon(K)=K}(-1)^{\left|K_{\epsilon}\right|} f_{K^{\prime}, J} e_{K^{\prime}, J} A \\
& =\sum_{\epsilon}^{\left|K_{\epsilon}^{\prime}\right|} \delta_{K^{\prime}, J}(-1)^{K_{\epsilon}^{\prime}} f_{K^{\prime}, J} e_{K^{\prime}, J} A=f_{J, J} e_{J, J} A=A
\end{aligned}
$$

and $(\mathrm{d})$ is proved.

We show that, for $J \subset \mathbf{I}$ such that $\epsilon(J)=J$ and $A, A^{\prime} \in \mathcal{K}\left(V_{J, D}\right)$, we have

$$
\left(\mathbf{d}_{J} A, \mathbf{d}_{J} A^{\prime}\right)_{J}=\left(A, A^{\prime}\right)_{J} .
$$

Using (b),(d) we have $\left(\mathbf{d}_{J} A, \mathbf{d}_{J} A^{\prime}\right)_{J}=\left(A, \mathbf{d}_{J} \mathbf{d}_{J} A^{\prime}\right)_{J}=\left(A, A^{\prime}\right)_{J}$ as desired.

38.11. We write $\mathbf{d}$ instead of $\mathbf{d}_{\mathbf{I}}$ and $($,$) instead of (,)_{\mathbf{I}}$. We call $\mathbf{d}$ the duality operator on character sheaves. If $A$ is a character sheaf on $D=V_{\mathbf{I}, D}$, then $(A, A)=1$ (where $A$ is regarded as an element of $\mathcal{K}(D)$ ) hence, by 38.10(e), we have $(\mathbf{d}(A), \mathbf{d}(A))=1$. Since $\mathbf{d}(A)$ is a $\mathbf{Z}$-linear combination of isomorphism classes of character sheaves (which form an orthonormal basis of $\mathcal{K}(D)$ for $($,$) ) it follows that$ $\mathbf{d}(A)= \pm A^{\prime}$ where $A^{\prime}$ is a well-defined character sheaf on $D$ (up to isomorphism). The sign can be described as follows. By 30.6(d) we can find a parabolic $P_{0}$ of $G^{0}$ such that $N_{D} P_{0} \neq \emptyset$ and a cuspidal character sheaf $A_{0}$ on $D_{0}:=N_{D} P_{0} / U_{P_{0}}$ such 
that $A$ is a direct summand of $\operatorname{ind}_{D_{0}}^{D}\left(A_{0}\right)$. We have $P_{0} \in \mathcal{P}_{J}$ where $J \subset \mathbf{I}, \epsilon(J)=J$. Then

$$
\mathbf{d}(A)=(-1)^{\left|J_{\epsilon}\right|} A^{\prime}
$$

Indeed, let $A_{0}^{b} \in C S\left(V_{D, J}\right)$ be the perverse sheaf corresponding to $A_{0}$ as in 30.3 . Then $e_{J^{\prime}, J} A_{0}^{b}=0$ for any $J^{\prime} \varsubsetneqq J$ such that $\epsilon\left(J^{\prime}\right)=J^{\prime}$; see 38.2. Hence $\mathbf{d}_{J}\left(A_{0}^{b}\right)=$ $(-1)^{\left|J_{\epsilon}\right|} A_{0}^{b}$. Now $A$ is a direct summand of $f_{J, \mathbf{I}} A_{0}^{b}$ and by $38.10(\mathrm{c})$ we have

$$
\mathbf{d} f_{J, \mathbf{I}} A_{0}^{b}=f_{J, \mathbf{I}} \mathbf{d}_{J} A_{0}^{b}=(-1)^{\left|J_{\epsilon}\right|} f_{J, \mathbf{I}} A_{0}^{b} .
$$

In $\mathcal{K}(D)$ we have $f_{J, \mathbf{I}} A_{0}^{b}=\sum_{k=1}^{k_{0}} n_{k} A_{k}$ where $A_{k}$ are distinct character sheaves on $D, n_{k} \in \mathbf{Z}_{>0}$ and $A_{1}=A$. We have $\mathbf{d}\left(A_{k}\right)=\iota_{k} A_{k}^{\prime}$ where $\iota_{k}= \pm 1$ and $A_{k}^{\prime}$ are distinct character sheaves on $D, n_{k} \in \mathbf{Z}_{>0}$ and $A_{1}=A$. We see that $\sum_{k} n_{k} \iota_{k} A_{k}^{\prime}=(-1)^{\left|J_{\epsilon}\right|} \sum_{k} n_{k} A_{k}$. Since $\left\{A_{k}\right\}$ and $\left\{A_{k}^{\prime}\right\}$ are parts of the same basis of $\mathcal{K}(D)$ we see that $\iota_{k} /(-1)^{\left|J_{\epsilon}\right|}>0$ for any $k$. Hence $\iota_{k}=(-1)^{\left|J_{\epsilon}\right|}$ for any $k$. In particular, this holds for $k=1$ and (a) follows.

Note that, by $38.10(\mathrm{~d}), A \mapsto A^{\prime}$ is an involution of the set of isomorphism classes of character sheaves on $D$.

38.12. The definition of the duality operator for character sheaves in 38.11 is entirely similar to that of a duality operator for representations of a reductive group over a finite field (given again by an alternating sum of compositions of a parabolic restriction and a parabolic induction) which was found by the author in 1977, who conjectured that it takes irreducibles to \pm irreducibles and is involutive. In 1977 I communicated this conjecture to C.W. Curtis and N. Kawanaka (see [Ka, p.412]); the conjecture was proved in $[\mathrm{Cu},[\mathrm{Al}]$ and in $\mathrm{Ka}$.

\section{Quasi-Rationality}

39.1. The main result of this section is Proposition 39.7 which gives a quasirationality property of representations of certain extensions of a Weyl group. This is needed to prove a key property of character sheaves (Corollary 39.8).

Let $W, I$ be a Weyl group ( $I$ is the set of simple reflections). We have canonically $W=\prod_{j \in J} W_{j}$ where $W_{j}$ is an irreducible Weyl group (with set of simple reflections $\left.I_{j}=I \cap W_{j}\right)$. We identify $W_{j}$ with a subgroup of $W$. Let $\mathrm{R}(W)=\bigcup_{w \in W} w I w^{-1}$. We have $\mathrm{R}(W)=\bigsqcup_{j \in J} \mathrm{R}\left(W_{j}\right)$. For any $j \in J$ the set $\mathrm{R}\left(W_{j}\right)$ is a single $W_{j^{-}}$ conjugacy class if $W_{j}$ is of type $A, D$ or $E$; it is a union of two $W_{j}$-conjugacy classes, otherwise. A subset $\mathcal{X}$ of $\mathrm{R}(W)$ is said to be special if $\mathcal{X}=\bigsqcup_{j \in J} \mathcal{X}_{j}$ where $\mathcal{X}_{j}$ is a $W_{j}$-conjugacy class in $\mathrm{R}\left(W_{j}\right)$. Clearly, a special subset of $\mathrm{R}(W)$ exists; we assume that a special subset $\mathcal{X}$ of $\mathrm{R}(W)$ is given. Let $\operatorname{Aut}(W, I, \mathcal{X})$ be the group of automorphisms of $W$ that preserve $I$ and $\mathcal{X}$.

Lemma 39.2. Let $\Gamma$ be a finite group. Let $\gamma \mapsto \rho_{\gamma}$ be a homomorphism $\Gamma \rightarrow$ $\operatorname{Aut}(W, I, \mathcal{X})$. Let $E$ be a simple $\mathbf{Q}[W]$-module such that $\operatorname{tr}\left(\rho_{\gamma}(w), E\right)=\operatorname{tr}(w, E)$ for any $\gamma \in \Gamma, w \in W$. Assume that either

(i) $|J|=1$, or

(ii) $\Gamma$ is an extension of a cyclic group by a cyclic group.

Then there exists a homomorphism $\Gamma \rightarrow \operatorname{Aut}_{\mathbf{Q}}(E), \gamma \mapsto t_{\gamma}$ such that $t_{\gamma}(w(e))=$ $\rho_{\gamma}(w) t_{\gamma}(e)$ for any $\gamma \in \Gamma, w \in W, e \in E$. 
$\Gamma$ acts on $J$ by $\gamma: j \mapsto \gamma(j)$ where $W_{\gamma(j)}=\rho_{\gamma}\left(W_{j}\right)$. We may identify $E=$ $\bigotimes_{j \in J} E_{j}$ as $\mathbf{Q}[W]$-modules where $E_{j}$ is a simple $\mathbf{Q}\left[W_{j}\right]$-module for any $j \in J$. From our assumption we see that for any $j \in J, \gamma \in \Gamma$ there exists a Q-linear isomorphism

(a) $H_{j}^{\gamma}: E_{j} \stackrel{\sim}{\longrightarrow} E_{\gamma(j)}$ with $H_{j}^{\gamma}\left(w_{j} e\right)=\rho_{\gamma}\left(w_{j}\right) H_{j}^{\gamma}(e)$ for all $e \in E_{j}, w_{j} \in W_{j}$; moreover, $H_{j}^{\gamma}$ is unique up to multiplication by an element of $\mathbf{Q}^{*}$. It follows that

(b) $H_{\gamma(j)}^{\gamma^{\prime}} H_{j}^{\gamma} \in \mathbf{Q}^{*} H_{j}^{\gamma^{\prime} \gamma}$ for $j \in J$ and $\gamma, \gamma^{\prime} \in \Gamma$.

For $\gamma \in \Gamma$ we define $\tilde{t}_{\gamma} \in \operatorname{Aut}_{\mathbf{Q}}(E)$ by

(c) $\tilde{t}_{\gamma}\left(\otimes_{j} e_{j}\right)=\bigotimes_{j} e_{j}^{\prime}$ where $e_{j} \in E_{j}$ and $e_{j}^{\prime}=H_{\gamma^{-1}(j)}^{\gamma}\left(e_{\gamma^{-1}(j)}\right) \in E_{j}$.

From definitions we have $\tilde{t}_{\gamma}(w(e))=\rho_{\gamma}(w) \tilde{t}_{\gamma}(e)$ for any $w \in W, e \in E$; moreover, for $\gamma, \gamma^{\prime} \in \Gamma$, the maps $\tilde{t}_{\gamma^{\prime} \gamma}, \tilde{t}_{\gamma^{\prime}} \tilde{t}_{\gamma}$ are equal up to a factor in $\mathbf{Q}^{*}$. Thus the maps $\tilde{t}_{\gamma}$ provide a homomorphism $\Gamma \rightarrow P G L(E)$ rather than a homomorphism $\Gamma \rightarrow G L(E)$.

We prove the lemma in the setup of (i). Replacing $\Gamma$ by its image under $\gamma \mapsto \rho_{g}$ we may assume that $\Gamma \subset \operatorname{Aut}(W, I, \mathcal{X})$ and $\gamma \mapsto \rho_{\gamma}$ is the inclusion. We form the semidirect product $W \Gamma$ with $W$ normal. It is enough to show that $E$ extends to a $W \Gamma$-module. We may assume that $\Gamma \neq\{1\}$. Then $W$ is of type $A_{n}(n \geq 2), D_{n}$ or $E_{6}$. If $\Gamma=\operatorname{Aut}(W, I, \mathcal{X})$, then $W \Gamma$ is itself a Weyl group, of type $A_{n} \times A_{1}, D_{2 n+1} \times A_{1}, B_{2 n}, F_{4}, E_{6} \times A_{1}$ for $W$ of type $A_{n}(n \geq 2), D_{2 n+1}(n \geq$ 2), $D_{2 n}(n \geq 3), D_{4}, E_{6}$ respectively and the desired result follows easily from the known properties of representations of such Weyl groups (in particular, from their rationality); the same applies if $W$ is of type $D_{4}$ and $|\Gamma|=2$ (in this case, $W \Gamma$ is a Weyl group of type $B_{4}$ ). In the only remaining case ( $W$ of type $D_{4},|\Gamma|=3$ ), the result follows by an argument in [L14, 3.2].

Next we prove the lemma in the setup of (ii). Now $\Gamma$ has two generators $a, c$ and relations $a^{M}=1, c^{N}=a^{u}, c a c^{-1}=a^{k}$ where $M, N, k$ are integers $\geq 1$ such that $k^{N}=1 \bmod M$ and $u \in \mathbf{N}$ satisfies $u k=k \bmod M$.

We consider separately 3 cases in increasing order of generality.

Case 1. Assume that $J$ is a single orbit of $a: J \rightarrow J$. We may identify $J=\mathbf{Z} / m$ so that $a(i)=i+1, c(i)=k i-r$ for $i \in \mathbf{Z} / m$; here $r \in \mathbf{N}$ is independent of $i$. Since $a^{M}=1: J \rightarrow J$ we see that $m$ divides $M$. For any $i$ we have $i+u=c^{N}(i)=k^{N} i-\left(1+k+k^{2}+\cdots+k^{N-1}\right) r$ in $\mathbf{Z} / m$. Since $k^{N}=1 \bmod M$ (hence $k^{N}=1 \bmod m$ ) we have $u+\left(1+k+k^{2}+\cdots+k^{N-1}\right) r=f m$ for some $f \in \mathbf{N}$. We set $c^{\prime}=a^{r} c$. Then $c^{\prime}(i)=k i$ for $i \in \mathbf{Z} / m$. Let $\Gamma^{\prime}$ be the subgroup of $\Gamma$ generated by $a^{m}, c^{\prime}$. In $\Gamma^{\prime}$ we have

$$
\left(a^{m}\right)^{M / m}=1, c^{N}=\left(a^{m}\right)^{f}, c^{\prime} a^{m} c^{-1}=\left(a^{m}\right)^{k} .
$$

Since $a^{m}(0)=0, c^{\prime}(0)=0$, the action of $\Gamma$ on $W$ restricts to an action of $\Gamma^{\prime}$ on $W_{0}$. Using the lemma (setup of (i)) for $W_{0}, E_{0}, \Gamma^{\prime}$ instead of $W, E, \Gamma$ we obtain a homomorphism $\Gamma^{\prime} \rightarrow \operatorname{Aut}_{\mathbf{Q}}\left(E_{0}\right)$ such that, denoting by $s_{0}, s_{1}$ the images of $a^{m}, c^{\prime}$ under this homomorphism, we have $s_{0}^{M / m}=1, s_{1}^{N}=s_{0}^{f}, s_{1} s_{0} s_{1}^{-1}=s_{0}^{k}, s_{0}(w(e))=$ $\rho_{a}^{m}(w) s_{0}(e), s_{1}(w(e))=\rho_{c^{\prime}}(w) s_{1}(e)$ for any $w \in W_{0}, e \in E_{0}$.

For $i \in \mathbf{Z} / m$, let $H_{i}^{a}: E_{i} \stackrel{\sim}{\longrightarrow} E_{i+1}$ be as in (a). Then $s_{2}:=H_{m-1}^{a} \ldots H_{1}^{a} H_{0}^{a}$ : $E_{0} \stackrel{\sim}{\longrightarrow} E_{0}$ satisfies $s_{2}(w(e))=\rho_{a}^{m}(w) s_{2}(e)$ for any $w \in W_{0}, e \in E_{0}$. Hence $s_{2}^{-1} s_{0}(w(e))=w\left(s_{2}^{-1} s_{0}(e)\right)$ for any $w \in W_{0}, e \in E_{0}$. By the absolute irreducibility of the $W_{0}$-module $E_{0}$ we see that $s_{2}^{-1} s_{0}: E_{0} \rightarrow E_{0}$ is a $\mathbf{Q}^{*}$-multiple of the identity 
map. Hence, replacing $H_{0}^{a}$ by a $\mathbf{Q}^{*}$-multiple, we may assume that $s_{2}=s_{0}$, that is, $H_{m-1}^{a} \ldots H_{1}^{a} H_{0}^{a}=s_{0}$. For any $h \in \mathbf{N}$ we set

$$
b_{h}=H_{h k-1}^{a} H_{h k-2}^{a} \ldots H_{1}^{a} H_{0}^{a} s_{1}\left(H_{h-1}^{a} \ldots H_{1}^{a} H_{0}^{a}\right)^{-1}: E_{h} \stackrel{\sim}{\longrightarrow} E_{k h}
$$

(there are $h k$ factors to the left of $s_{1}$ and $h$ factors to the right of $s_{1}$ ). For $h \geq 1$ we have

(d) $b_{h}=H_{h k-1}^{a} H_{h k-2}^{a} \ldots H_{h k-k}^{a} b_{h-1}\left(H_{h-1}^{a}\right)^{-1}$.

We show that $b_{h+m}=b_{h}$ for $h \in \mathbf{N}$. We argue by induction on $h$. Assume first that $h=0$. We must show that $H_{m k-1}^{a} H_{m k-2}^{a} \ldots H_{1}^{a} H_{0}^{a} s_{1}\left(H_{m-1}^{a} \ldots H_{1}^{a} H_{0}^{a}\right)^{-1}=s_{1}$; this is a reformulation of the already known equality $s_{0}^{k} s_{1} s_{0}^{-1}=s_{1}$. Assume next that $h \geq 1$. Using (d) twice and the induction hypothesis we have

$$
\begin{aligned}
b_{h+m} & =H_{h k+m k-1}^{a} H_{h k+m k-2}^{a} \ldots H_{h k+m k-k}^{a} b_{h+m-1}\left(H_{h+m-1}^{a}\right)^{-1} \\
& =H_{h k-1}^{a} H_{h k-2}^{a} \ldots H_{h k-k}^{a} b_{h-1}\left(H_{h-1}^{a}\right)^{-1}=b_{h},
\end{aligned}
$$

as desired. We see that $b_{h}$ depends only on the image of $h$ in $\mathbf{Z} / m$.

We set $k=k^{N-1}$. Then $k k^{\prime}=1 \bmod M$. Define $t_{a}, t_{c^{\prime}} \in \operatorname{Aut}_{\mathbf{Q}}(E)$ by $t_{a}\left(\bigotimes_{i \in \mathbf{Z} / m} e_{i}\right)=\bigotimes_{i \in \mathbf{Z} / m} e_{i}^{\prime}$ where $e_{i} \in E_{i}$ and $e_{i}^{\prime}=H_{i-1}^{a}\left(e_{i-1}\right) \in E_{i}, t_{c^{\prime}}\left(\bigotimes_{i \in \mathbf{Z} / m} e_{i}\right)$ $=\bigotimes_{i \in \mathbf{Z} / m} e_{i}^{\prime \prime}$ where $e_{i} \in E_{i}$ and $e_{i}^{\prime \prime}=b_{i k^{\prime}}\left(e_{i k^{\prime}}\right) \in E_{i k^{\prime} k}=E_{i}$. From definitions we have $t_{a}(w(e))=\rho_{a}(w) t_{a}(e), t_{c^{\prime}}(w(e))=\rho_{c^{\prime}}(w) t_{c^{\prime}}(e)$ for any $w \in W, e \in E$.

We have $t_{c^{\prime}} t_{a} t_{c^{\prime}}^{-1}=t_{a}^{k}$. This follows from the identity

$$
b_{h k^{\prime}} H_{h k^{\prime}-1}^{a} b_{h k^{\prime}-1}^{-1}=H_{h-1}^{a} H_{h-2}^{a} \ldots H_{h-k}^{a}: E_{h-k} \rightarrow E_{h}
$$

for $h=0,1, \ldots, m-1$ (here $b_{-1}$ is taken to be $b_{m-1}$ ); an equivalent identity is

$$
b_{i} H_{i-1}^{a} b_{i-1}^{-1}=H_{i k-1}^{a} H_{i k-2}^{a} \ldots H_{i k-k}^{a}: E_{i k-k} \rightarrow E_{i k}
$$

for $i=0,1, \ldots, m-1$, which is the same as (d).

We have $t_{a}^{M}=1$. This follows from the identity $H_{i+M-1}^{a} \ldots H_{i+1}^{a} H_{i}^{a}=1: E_{i} \rightarrow$ $E_{i}$ for $i=0,1, \ldots, m-1$ which is equivalent to the known equality $s_{0}^{M / m}=1$.

We show that

(e) $t_{c^{\prime}}^{N}=t_{a}^{m f}$.

This follows from the identity

$$
b_{i k^{\prime}} b_{i k^{\prime 2}} \ldots b_{i k^{\prime N}}=H_{i-1}^{a} H_{i-2}^{a} \ldots H_{i-m f}^{a} \quad \text { for } i=0,1, \ldots, m-1
$$

or equivalently

$$
b_{i k^{N-1}} b_{i k^{N-2}} \ldots b_{i}=H_{i-1}^{a} H_{i-2}^{a} \ldots H_{i-m f}^{a} \quad \text { for } i=0,1, \ldots, m-1 .
$$

This is the same as

$$
H_{i k^{N}-1}^{a} H_{i k^{N}-2}^{a} \ldots H_{1}^{a} H_{0}^{a} s_{1}^{N}\left(H_{i-1}^{a} \ldots H_{1}^{a} H_{0}^{a}\right)^{-1}=H_{i-1}^{a} H_{i-2}^{a} \ldots H_{i-m f}^{a}
$$

(with $i k^{N}$ factors to the left of $s_{1}^{N}$ ). We have $i k^{N}-i=i M l$ for some $l \in \mathbf{N}$. From $s_{0}^{M / m}=1$ we deduce $s_{0}^{i M l / m}=1$ hence $H_{i k^{N}-1}^{a} H_{i k^{N}-2}^{a} \ldots H_{i}^{a}=1\left(\right.$ with $i k^{N}-i$ factors). Since $s_{1}^{N}=s_{0}^{f}$, it remains to show that

$$
H_{i-1}^{a} \ldots H_{1}^{a} H_{0}^{a} s_{0}^{f}\left(H_{i-1}^{a} \ldots H_{1}^{a} H_{0}^{a}\right)^{-1}=H_{i-1}^{a} H_{i-2}^{a} \ldots H_{i-m f}^{a} .
$$

If $f=0$, this is obvious. Assume now that $f \geq 1$. Then $i-m f<0$ and we see that it is enough to show $s_{0}^{f}\left(H_{i-1}^{a} \ldots H_{1}^{a} H_{0}^{a}\right)^{-1}=H_{-1}^{a} H_{-2}^{a} \ldots H_{i-m f}^{a}$, that is, $s_{0}^{f}=H_{-1}^{a} H_{-2}^{a} \ldots H_{i-m f}^{a}\left(H_{i-1}^{a} \ldots H_{1}^{a} H_{0}^{a}\right)$. This can be rewritten in the form $s_{0}^{f}=$ 
$H_{-1}^{a} H_{-2}^{a} \ldots H_{i-m f}^{a} H_{i-m f-1}^{a} \ldots H_{1-m f}^{a} H_{-m f}^{a}$ which follows from $s_{0}=H_{m-1}^{a} \ldots$ $H_{1}^{a} H_{0}^{a}$. This proves (e).

We set $t_{c}=t_{a}^{-r} t_{c^{\prime}} \in \operatorname{Aut}_{\mathbf{Q}}(E)$. From definitions we have $t_{c}(w(e))=\rho_{c}(w) t_{c}(e)$ for any $w \in W, e \in E$.

We have $t_{c} t_{a} t_{c}^{-1}=t_{a}^{k}$. This follows from the identity $t_{c^{\prime}} t_{a} t_{c^{\prime}}^{-1}=t_{a}^{k}$.

We have $t_{c}^{N}=t_{a}^{u}$. Indeed,

$$
\left(t_{a}^{-r} t_{c^{\prime}}\right)^{N}=t_{a}^{-r\left(1+k+k^{2}+\cdots+k^{N-1}\right)} t_{c^{\prime}}^{N}=t_{a}^{-m f+u} t_{a}^{m f}=t_{a}^{u} .
$$

We see that $t_{a}, t_{c} \in \operatorname{Aut}_{\mathbf{Q}}(E)$ satisfy the relations of $\Gamma$, hence they define a homomorphism $\Gamma \rightarrow \operatorname{Aut}_{\mathbf{Q}}(E)$. This has the required properties.

Case 2. Assume that $J$ is a single $\Gamma$-orbit. Let $\langle a\rangle$ be the subgroup of $\Gamma$ generated by $a$. If $X$ is an $\langle a\rangle$-orbit in $J$, then $c X$ is again an $\langle a\rangle$-orbit. (We must show that, if $j \in X$ and $i \geq 1$, then $c a^{i} j, c j$ are in the same $\langle a\rangle$-orbit. But $c a^{i} j=a^{i k} c j$.) Hence $c^{h} X$ is an $\langle a\rangle$-orbit in $J$ for any $h \in \mathbf{N}$. We can find an integer $z \geq 1$ such that $X, c X, \ldots, c^{z-1} X$ are distinct and $c^{z} X=X$. (Clearly, $z$ is a divisor of $N$.) Hence the notation $X_{h}=c^{h} X$ for $h \in \mathbf{Z} / z$ is meaningful. Now $X_{0}, X_{1}, \ldots, X_{z-1}$ are distinct. The union $\bigcup_{h \in[0, z-1]} X_{h}$ is $c$-stable and $a$-stable, hence it is equal to $J$ (by our assumption on $J)$. We see that $|J|=|X| z$. For $h \in \mathbf{Z} / z$ we set $W^{h}=\prod_{j \in X_{h}} W_{j}$, $E^{h}=\bigotimes_{j \in X_{h}} E_{j}$. We have naturally $W=\prod_{h \in[0, z-1]} W^{h}, E=\bigotimes_{h \in[0, z-1]} E^{h}$. Let $\Gamma^{\prime \prime}$ be the subgroup of $\Gamma$ generated by $a, c^{z}$. In $\Gamma^{\prime \prime}$ we have

$$
a^{M}=1,\left(c^{z}\right)^{N / z}=a^{u}, c^{z} a c^{-z}=a^{k^{z}} .
$$

Since $a\left(X_{0}\right)=X_{0}, c^{z}\left(X_{0}\right)=X_{0}$, the action of $\Gamma$ on $W$ restricts to an action of $\Gamma^{\prime \prime}$ on $W^{0}$. Using case 1 for $W^{0}, E^{0}, \Gamma^{\prime \prime}$ instead of $W, E, \Gamma$ we obtain a homomorphism $\Gamma^{\prime \prime} \rightarrow \operatorname{Aut}_{\mathbf{Q}}\left(E^{0}\right)$ such that, denoting by $S_{0}, S_{1}$ the images of $a, c^{z}$ under this homomorphism, we have $S_{0}^{M}=1, S_{1}^{N / z}=S_{0}^{u}, S_{1} S_{0} S_{1}^{-1}=S_{0}^{k^{z}}, S_{0}(w(e))=\rho_{a}(w) S_{0}(e)$, $S_{1}(w(e))=\rho_{c}^{z}(w) S_{1}(e)$ for any $w \in W^{0}, e \in E^{0}$.

For $h \in \mathbf{Z} / z$ there exists a Q-linear isomorphism $K_{h}: E^{h} \stackrel{\sim}{\longrightarrow} E^{h+1}$ with $K_{h}(w e)=\rho_{c}(w) K_{h}(e)$ for all $e \in E^{h}, w \in W^{h}$ (note that $\rho_{c}(w) \in W^{h+1}$ ); moreover, $K_{h}$ is unique up to multiplication by an element of $\mathbf{Q}^{*}$. (For example we can take $K_{h}$ of the form $\bigotimes_{j \in X_{h}} H_{j}^{c}$ where $H_{j}^{c}: E_{j} \stackrel{\sim}{\rightarrow} E_{c j}$ are as in (a).) Then $S_{2}:=K_{z-1} \ldots K_{1} K_{0}: E^{0} \stackrel{\sim}{\longrightarrow} E^{0}$ satisfies $S_{2}(w(e))=\rho_{c}^{z}(w) S_{2}(e)$ for any $w \in W^{0}, e \in E^{0}$. Hence $S_{2}^{-1} S_{1}(w(e))=w\left(S_{2}^{-1} S_{1}(e)\right)$ for any $w \in W^{0}, e \in E^{0}$. By the absolute irreducibility of the $W^{0}$-module $E^{0}$ we see that $S_{2}^{-1} S_{1}: E^{0} \rightarrow E^{0}$ is a $\mathbf{Q}^{*}$-multiple of the identity map. Hence, replacing $K_{0}$ by a $\mathbf{Q}^{*}$-multiple, we may assume that $S_{2}=S_{1}$ that is, $K_{z-1} \ldots K_{1} K_{0}=S_{1}$.

For any $h \in \mathbf{N}$ we define $\beta_{-h} \in \operatorname{Aut}_{\mathbf{Q}}\left(E^{-h}\right)$ by

$$
\beta_{-h}=K_{-h}^{-1} \ldots K_{-2}^{-1} K_{-1}^{-1} S_{0}^{k^{h}} K_{-1} K_{-2} \ldots K_{-h}
$$

(for $h=0$ this is interpreted as $\beta_{0}=S_{0}$ ). We show that $\beta_{-h-z}=\beta_{-h}$. We argue by induction on $h$. For $h=0$ we must verify that

$$
K_{-z}^{-1} \ldots K_{-2}^{-1} K_{-1}^{-1} S_{0}^{k^{z}} K_{-1} K_{-2} \ldots K_{-z}=S_{0} \text {; }
$$

this follows from the known equality $S_{1}^{-1} S_{0}^{k^{z}} S_{1}=S_{0}$. For $h \geq 1$ we have, using the induction hypothesis,

$$
\beta_{-h-z}=K_{-h-z}^{-1} \beta_{-h-z+1}^{k} K_{-h-z}=K_{-h}^{-1} \beta_{-h+1}^{k} K_{-h}=\beta_{-z} .
$$


We see that $\beta_{-h}$ depends only on the image of $h$ in $\mathbf{Z} / z$. Define $t_{a}, t_{c} \in \operatorname{Aut}_{\mathbf{Q}}(E)$ by

$\tau_{a}\left(\bigotimes_{h \in \mathbf{Z} / z} e_{h}\right)=\bigotimes_{h \in \mathbf{Z} / z} e_{h}^{\prime}$ where $e_{h} \in E^{h}$ and $e_{h}^{\prime}=\beta_{h}\left(e_{h}\right) \in E^{h}$,

$\tau_{c}\left(\otimes_{h \in \mathbf{Z} / z} e_{h}\right)=\bigotimes_{h \in \mathbf{Z} / z} e_{h}^{\prime \prime}$ where $e_{h} \in E^{h}$ and $e_{h}^{\prime \prime}=K_{h-1}\left(e_{h-1}\right) \in E^{h}$.

From definitions we have $t_{a}(w(e))=\rho_{a}(w) t_{a}(e), t_{c}(w(e))=\rho_{c}(w) t_{c}(e)$ for any $w \in W, e \in E$.

We have $t_{c} t_{a} t_{c}^{-1}=t_{a}^{k}$. This follows from the identity $\beta_{h-1}=K_{h-1}^{-1} \beta_{h}^{k} K_{h-1}$ for $h \in \mathbf{Z} / z$.

We have $t_{a}^{M}=1$. This follows from the identity $\beta_{-h}^{M}=1$ for any $h \in \mathbf{N}$. An equivalent statement is $K_{-h}^{-1} \ldots K_{-2}^{-1} K_{-1}^{-1} S_{0}^{k^{h} M} K_{-1} K_{-2} \ldots K_{-h}=1$ which follows from $S_{0}^{M}=1$.

We show that $t_{c}^{N}=t_{a}^{u}$. It is enough to show that $K_{-h-1} K_{-h-2} \ldots K_{-h-N}=$ $b_{-h}^{u}: E_{-h} \rightarrow E_{-h}$ for $h=0,1, \ldots, z-1$. An equivalent statement is

$$
\left(K_{-h}^{-1} \ldots K_{-1}^{-1}\right)\left(K_{-1} K_{-2} \ldots K_{-N}\right)\left(K_{-1} \ldots K_{-h}\right)=K_{-h}^{-1} \ldots K_{-1}^{-1} S_{0}^{k^{h} u} K_{-1} \ldots K_{-h} .
$$

The left-hand side is $\left(K_{-h}^{-1} \ldots K_{-1}^{-1}\right) S_{1}^{N / z}\left(K_{-1} \ldots K_{-h}\right)$. It is enough to show that $S_{1}^{N / z}=S_{0}^{k^{h} u}$ or that $S_{0}^{u}=S_{0}^{k^{h} u}$ for $h=0,1, \ldots, z-1$. Since $S_{0}^{M}=1$, it is enough to show that $u=k^{h} u \bmod M$. This follows from $u k=u \bmod M$.

We see that $t_{a}, t_{c} \in \operatorname{Aut}_{\mathbf{Q}}(E)$ satisfy the relations of $\Gamma$, hence they define a homomorphism $\Gamma \rightarrow \operatorname{Aut}_{\mathbf{Q}}(E)$. This has the required properties.

Case 3. We now consider the general case. For any $\Gamma$-orbit $Y$ on $J$ we set $W^{Y}=$ $\prod_{j \in Y} W_{j}, E^{Y}=\bigotimes_{j \in Y} E_{j}$. We have naturally $W=\prod_{Y} W^{Y}, E=\bigotimes_{Y} E^{Y}$ where $Y$ runs over the $\Gamma$-orbits in $J$. Now the $\Gamma$-action on $W$ restricts to a $\Gamma$-action on $W^{Y}$ for each $Y$. Using case 2 for $W^{Y}, E^{Y}, \Gamma$ instead of $W, E, \Gamma$ we obtain for each $Y$ a homomorphism $\Gamma \rightarrow \operatorname{Aut}_{\mathbf{Q}}\left(E^{Y}\right)$. We define a homomorphism $\Gamma \rightarrow \operatorname{Aut}_{\mathbf{Q}}(E)$ by $\gamma: \bigotimes_{Y} e_{Y} \mapsto \bigotimes_{Y}\left(\gamma\left(e_{Y}\right)\right)$; here $e_{Y} \in E^{Y}$. This has the required properties.

This completes the proof in the setup of (ii). The lemma is proved.

39.3. Let $\Gamma$ be a finite group. Assume that $\Gamma$ is a semidirect product of a normal subgroup $\Gamma^{\prime}$ with a cyclic group of order $n$ with generator $b$. Let $\overline{\mathfrak{U}}$ be an algebraic closed field of characteristic 0 . Let $E$ be a simple $\overline{\mathfrak{U}}[\Gamma]$-module which is isotypical as a $\overline{\mathfrak{U}}\left[\Gamma^{\prime}\right]$-module. We show that (a) $E$ is simple as a $\overline{\mathfrak{U}}\left[\Gamma^{\prime}\right]$-module.

There exists a simple $\overline{\mathfrak{U}}\left[\Gamma^{\prime}\right]$-module $E^{\prime}$ such that, setting $V=\operatorname{Hom}_{\overline{\mathfrak{U}}\left[\Gamma^{\prime}\right]}\left(E^{\prime}, E\right)$ we have $V \otimes E^{\prime} \stackrel{\sim}{\longrightarrow} E, f \otimes e^{\prime} \mapsto f\left(e^{\prime}\right)$. For any $\gamma^{\prime} \in \Gamma^{\prime}$ we have $\operatorname{tr}\left(b \gamma^{\prime} b^{-1}, E^{\prime}\right)=$ $\operatorname{tr}\left(\gamma^{\prime}, E^{\prime}\right)$. Hence there exists $\xi \in \operatorname{Aut}_{\overline{\mathfrak{U}}}\left(E^{\prime}\right)$ such that $\xi\left(\gamma^{\prime} e^{\prime}\right)=\left(b \gamma^{\prime} b^{-1}\right) \xi\left(e^{\prime}\right)$ for $e^{\prime} \in E^{\prime}, w^{\prime} \in \Gamma^{\prime}$. Then $\xi^{n} \in \operatorname{Aut}_{\overline{\mathfrak{U}}}\left(E^{\prime}\right)$ commutes with the $\Gamma^{\prime}$-action hence it is a scalar on $E^{\prime}$. Replacing $\xi$ by an $\overline{\mathfrak{U}}^{*}$-multiple we can assume that $\xi^{n}=1$. Then $E^{\prime}$ becomes a $\overline{\mathfrak{U}}[\Gamma]$-module with $b$ acting as $\xi$. Define $\eta: V \rightarrow V$ by $f \mapsto \eta(f)$ where $\eta(f)\left(e^{\prime}\right)=b f\left(\xi^{-1}\left(e^{\prime}\right)\right)$ for $e^{\prime} \in E^{\prime}$. We regard $V$ as a $\overline{\mathfrak{U}}[\Gamma]$-module in which $\Gamma^{\prime}$ acts trivially and $b$ acts as $\eta$. Then the isomorphism $V \otimes E^{\prime} \stackrel{\sim}{\longrightarrow} E$ considered above is an isomorphism of $\overline{\mathfrak{U}}[\Gamma]$-modules. Since $E$ is simple it follows that $V$ is a simple $\overline{\mathfrak{U}}[\Gamma]$-module. Since $\Gamma$ acts on $V$ through a cyclic quotient, we see that $\operatorname{dim} V=1$. It follows that $E \cong E^{\prime}$ as a $\overline{\mathfrak{U}}\left[\Gamma^{\prime}\right]$-module; (a) follows.

39.4. Let $W, I, \mathcal{X}$ be as in 39.1. Let $\Gamma$ be a finite group with generators $a, c$ and relations $a^{M}=1, c^{N}=1, c a c^{-1}=a^{k}$ where $M, N, k$ are integers $\geq 1$ such that $k^{N}=1 \bmod M$. Let $\langle a\rangle$ (resp. $\langle c\rangle$ ) be the subgroup of $\Gamma$ generated by $a$ (resp. 
$c)$. Let $\gamma \mapsto \rho_{\gamma}$ be a homomorphism $\Gamma \rightarrow \operatorname{Aut}(W, I, \mathcal{X})$. We form the semidirect product $W \Gamma, W\langle a\rangle$ with $W$ normal. Note that $W\langle a\rangle$ is a subgroup of $W \Gamma$.

Lemma 39.5. In the setup of 39.4 let $E$ be a simple $\overline{\mathfrak{U}}[W \Gamma]$-module. Assume that either $c x c^{-1}=x$ for any $x \in\langle a\rangle$, or $c x c^{-1}=x^{-1}$ for any $x \in\langle a\rangle$. Let $x \in\langle a\rangle$. There exists $\zeta$, a root of 1 in $\overline{\mathfrak{U}}$, such that $\operatorname{tr}(w x c, E) \in \zeta \mathbf{Z}$ for any $w \in W$.

Let $W^{\prime}=W\langle a\rangle$. We can write canonically $E=\bigoplus_{t \in T} E^{t}$ where $E^{t}$ are isotypical $\overline{\mathfrak{U}}\left[W^{\prime}\right]$-modules. Now $\Gamma$ acts on $T$ by $\gamma E^{t}=E_{\gamma(t)}$. This action is transitive since $E$ is simple as a $\overline{\mathfrak{U}}[W \Gamma]$-module. If $c(t) \neq t$ for any $t \in T$, then for any $w \in$ $W$, wxc $: E \rightarrow E$ permutes the summands $E^{t}$ and no summand is stable, hence $\operatorname{tr}(w x c, E)=0$. In this case the lemma is clear. Thus we may assume that $c(t)=t$ for some $t \in T$. Then $c E^{t}=E^{t}$, hence $E^{t}$ is a $\overline{\mathfrak{U}}[W \Gamma]$-submodule of $E$. Since $E$ is simple, we have $E=E^{t}$. Thus, $E$ is isotypical as a $\overline{\mathfrak{U}}\left[W^{\prime}\right]$-module. Using 39.3(a) for $W \Gamma, W^{\prime},\langle c\rangle$ instead of $\Gamma, \Gamma^{\prime}, C$ we see that $E$ is simple as a $\overline{\mathfrak{U}}\left[W^{\prime}\right]$-module.

We can write canonically $E=\bigoplus_{h \in H} E_{h}$ where $E_{h}$ are isotypical $\overline{\mathfrak{U}}[W]$-modules. Now $\Gamma$ acts on $H$ by $\gamma E_{h}=E_{\gamma(h)}$. This action is transitive since $E$ is simple as a $\mathfrak{U}[W \Gamma]$-module. The restriction of this action to $\langle a\rangle$ is also transitive since $E$ is simple as a $\overline{\mathfrak{U}}\left[W^{\prime}\right]$-module. For $h \in H$ let $\langle a\rangle_{h}$ be the stabilizer of $h$ in $\langle a\rangle$. Let $W_{h}^{\prime}=W\langle a\rangle_{h} \subset W^{\prime}$. Then $E_{h}$ is a $W_{h}^{\prime}$-submodule of $E$ and the $W^{\prime}$-module $E$ is induced by the $W_{h}^{\prime}$-module $E_{h}$. Since $E$ is simple as a $W^{\prime}$-module, it follows that $E_{h}$ is simple as a $W_{h}^{\prime}$-module. Using $39.3\left(\right.$ a) for $W_{h}^{\prime}, W, E_{h}$ instead of $\Gamma, \Gamma^{\prime}, E$, we see that $E_{h}$ is simple as a $W$-module.

For $h \in H$ let $\Gamma_{h}=\{\gamma \in \Gamma ; \gamma(h)=h\}$. Then $E_{h}$ is a $W \Gamma_{h}$-submodule of $E$. Note that $\Gamma_{h}$ is an extension of a cyclic group (the image of $\Gamma_{h}$ under $\Gamma \rightarrow \Gamma /\langle a\rangle$ ) by a cyclic group (the intersection $\Gamma_{h} \cap\langle a\rangle$ ). Let $H_{0}=\{h \in H ; x c(h)=h\}$. For any $w \in W$ we have $\operatorname{tr}(w x c, E)=\sum_{h \in H_{0}} \operatorname{tr}\left(w x c, E_{h}\right)$. In particular, if $H_{0}=\emptyset$, then $\operatorname{tr}(w x c, E)=0$ so that the lemma is clear in this case. Thus we may assume that $H_{0} \neq \emptyset$. Let $h \in H_{0}$. We can find a simple $\mathbf{Q}[W]$-submodule $E_{h}^{0}$ of $E^{h}$ such that $E^{h}=\overline{\mathfrak{U}} \otimes_{\mathbf{Q}} E_{h}^{0}$ as $\overline{\mathfrak{U}}[W]$-modules. By Lemma 39.2 applied to $W, \Gamma_{h}, E_{h}^{0}$ instead of $W, \Gamma, E$ we see that the $\mathbf{Q}[W]$-structure on $E_{h}^{0}$ extends to a $\mathbf{Q}\left[W \Gamma_{h}\right]$-module structure. (The hypotheses of that lemma are satisfied since the $\overline{\mathfrak{U}}[W]$-module structure on $E_{h}$ extends to a $\overline{\mathfrak{U}}$-module structure.) For $\gamma \in \Gamma_{h}$ let $t_{\gamma} \in \operatorname{Aut}_{\mathbf{Q}}\left(E_{h}^{0}\right)$ be the action of $\gamma$ in this $\mathbf{Q}\left[W \Gamma_{h}\right]$-module. By extension of scalars, $t_{\gamma}$ defines an element $\tilde{t}_{\gamma} \in \operatorname{Aut}_{\mathbf{Q}}\left(E_{h}\right)$. For $e \in E_{h}, w \in W$ we have $\tilde{t}_{\gamma}(w e)=\left(\gamma w \gamma^{-1}\right)\left(\tilde{t}_{\gamma}(e)\right)$. Hence $\gamma^{-1}\left(\tilde{t}_{\gamma}(w e)\right)=w \gamma^{-1} \tilde{t}_{g}(e)$. Thus $\gamma^{-1} \tilde{t}_{\gamma}: E_{h} \rightarrow E_{h}$ commutes with the action of $W$. Since $E_{h}$ is simple we see that $\gamma^{-1} \tilde{t}_{\gamma}: E_{h} \rightarrow E_{h}$ is a scalar $\lambda_{h}(\gamma) \in \overline{\mathfrak{U}}^{*}$. Thus $\tilde{t}_{\gamma}=\lambda_{h}(\gamma) \gamma: E_{h} \rightarrow E_{h}$. Clearly, $\gamma \mapsto \lambda_{h}(\gamma)$ is a homomorphism $\Gamma_{h} \rightarrow \overline{\mathfrak{U}}^{*}$. For $w \in W$ we have $w x c=\lambda_{h}(x c)^{-1} w \tilde{t}_{x c}: E_{h} \rightarrow E_{h}$, hence

$$
\operatorname{tr}\left(w x c, E_{h}\right)=\lambda_{h}(x c)^{-1} \operatorname{tr}\left(w \tilde{t}_{x c}, E_{h}\right)=\lambda_{x c}^{-1} \operatorname{tr}\left(w t_{x c}, E_{h}^{0}\right) .
$$

Note that $\operatorname{tr}\left(w t_{x c}, E_{h}^{0}\right) \in \mathbf{Z}$ since $E_{h}^{0}$ is a $\mathbf{Q}\left[W \Gamma_{h}\right]$-module. We deduce

$$
\operatorname{tr}(w x c, E)=\sum_{h \in H_{0}} \lambda_{h}(x c)^{-1} \operatorname{tr}\left(w t_{x c}, E_{h}^{0}\right) .
$$

Let $H_{1}=\left\{h \in H_{0} ; \operatorname{tr}\left(w^{\prime} t_{x c}, E_{h}^{0}\right) \neq 0\right.$ for some $\left.w^{\prime} \in W\right\}$. Then, clearly,

$$
\operatorname{tr}(w x c, E)=\sum_{h \in H_{1}} \lambda_{h}(x c)^{-1} \operatorname{tr}\left(w t_{x c}, E_{h}^{0}\right) .
$$


Since $\lambda_{h}(x c)$ is a root of 1 , it is enough to verify the following statement: If $h, h^{\prime} \in$ $H_{1}$, then $\lambda_{h}(x c)= \pm \lambda_{h^{\prime}}(x c)$. Since $\langle a\rangle$ acts transitively on $H$, we can find $y \in\langle a\rangle$ such that $h=y h^{\prime}$. We have $y E_{h^{\prime}}=E_{h}, y \Gamma_{h^{\prime}} y^{-1}=\Gamma_{h}$. Also, for any $w^{\prime} \in W$ we have

$$
\operatorname{tr}\left(w^{\prime} x c, E_{h^{\prime}}\right)=\operatorname{tr}\left(y w^{\prime} x c y^{-1}, E_{h}\right)=\operatorname{tr}\left(y w^{\prime} y^{-1} y x c y^{-1}, E_{h}\right),
$$

hence

$$
\lambda_{h^{\prime}}(x c) \operatorname{tr}\left(w^{\prime} x c, E_{h^{\prime}}^{0}\right)=\lambda_{h}\left(y x c y^{-1}\right) \operatorname{tr}\left(y w^{\prime} y^{-1} w^{\prime} x c y^{-1}, E_{h}^{0}\right) .
$$

We take here $w^{\prime} \in W$ such that $\operatorname{tr}\left(w^{\prime} x c, E_{h^{\prime}}^{0}\right) \neq 0$. Dividing by $\operatorname{tr}\left(w^{\prime} x c, E_{h^{\prime}}^{0}\right)$ we deduce $\lambda_{h^{\prime}}(x c) \in \mathbf{Q} \lambda_{h}\left(y x c y^{-1}\right)$. Since $\lambda_{h^{\prime}}(x c), \lambda_{h}\left(y x c y^{-1}\right)$ are roots of 1 , it follows that $\lambda_{h^{\prime}}(x c)= \pm \lambda_{h}\left(y x c y^{-1}\right)$. Thus it is enough to show that $\lambda_{h}(x c)=$ $\pm \lambda_{h}\left(y x c y^{-1}\right)$ or that $\lambda_{h}\left(c^{-1} x^{-1} y x c y^{-1}\right)= \pm 1$ or that $\lambda_{h}\left(c^{-1} y c y^{-1}\right)= \pm 1$. More generally, we will verify the following statement: If $h \in H_{0}, y \in\langle a\rangle, c^{-1} y c y^{-1} \in$ $\langle a\rangle_{h}$, then $\lambda_{h}\left(c^{-1} y c y^{-1}\right)= \pm 1$. If $c y=y c=1$, this is obvious. Therefore, we may assume that $c y^{\prime}=y^{\prime-1} c$ for any $y^{\prime} \in\langle a\rangle$.

Let $u \in\langle a\rangle_{h}$. Since $u, x c$ belong to $\Gamma_{h}$ we have $\lambda_{h}\left((x c)^{-1} u x c u^{-1}\right)=1$, that is, $\lambda_{h}\left(c^{-1} u c u^{-1}\right)=1$ (we use $u x=x u$ ). But $c^{-1} u c=u^{-1}$. Hence $\lambda_{h}\left(u^{-2}\right)=1$, that is, $\lambda_{h}(u)^{-2}=1$ and $\lambda_{h}(u)= \pm 1$. We apply this with $u=c^{-1} y_{1} c y_{1}^{-1} \in\langle a\rangle_{h}$. We see that $\lambda_{h}\left(c^{-1} y_{1} c y_{1}^{-1}\right)= \pm 1$. The lemma is proved.

39.6. Let $W, I, \mathcal{X}$ are as in 39.1. We assume that $(W, I)$ is irreducible. Let $\tilde{I}=$ $I \sqcup\{\omega\}$ where $\omega$ is a symbol. We define a map $\pi: \tilde{I} \rightarrow W$ as follows: $\pi(s)=s$ if $s \in I$ and $\pi(\omega)$ is the unique reflection of maximal length in $\mathcal{X}$. The restriction of $\pi$ to $\tilde{I}$ is injective if $|I| \geq 2$; if $|I|=1$, it maps both elements of $\tilde{I}$ to the unique element of $I$. Let $\Omega$ be the group of all permutations $\sigma: \tilde{I} \stackrel{\sim}{\longrightarrow} \tilde{I}$ such that there exist $w \in W$ with $w \pi(\sigma) w^{-1}=\pi(\sigma(x))$ for all $x \in \tilde{I}$. Then $\sigma \mapsto\left[w_{1} \mapsto\right.$ $\left.w w_{1} w^{-1}\right]$ is a homomorphism of $\Omega$ into $\operatorname{Inn}(W)$, the group of inner automorphisms of $W$. Let $K$ be a subset of $\tilde{I}$ such that $K \neq \tilde{I}$. Then $\pi$ restricts to a bijection $K \stackrel{\sim}{\longrightarrow} \pi(K)$. Let $\Omega^{K}=\{\sigma \in \Omega ; \sigma(K)=K\}$. Let $W^{(K)}$ be the subgroup of $W$ generated by $\pi(K)$. From the theory of affine Weyl groups we see that $W^{(K)}$ is a (finite) Coxeter group on the generators $\pi(K)$. We have canonically $W^{(K)}=$ $\prod_{z \in Z} W_{z}^{(K)}$ where $W_{z}^{(K)}$ is an irreducible Weyl group with set of simple reflections $K_{z}=K \cap W_{z}^{(K)}$. For $z \in Z$ we set $\mathcal{X}_{z}^{K}=\mathrm{R}\left(W_{z}^{(K)}\right)$ if $\mathrm{R}\left(W_{z}^{(K)}\right)$ is a single $W_{z}^{(K)}$ conjugacy class and $\mathcal{X}_{z}^{K}=\mathcal{X} \cap W_{z}^{(K)}$ if $\mathrm{R}\left(W_{z}^{(K)}\right)$ is a union of two $W_{z}^{(K)}$-conjugacy classes; in any case, $\mathcal{X}_{z}^{K}$ is a single $W_{z}^{(K)}$-conjugacy class in $\mathrm{R}\left(W_{z}^{(K)}\right)$. (We use the following fact: If $s \in I$ is such that $s \pi(\omega)$ has order $\geq 4$, then $s, \pi(\omega)$ are not conjugate under $W$.) Then $\mathcal{X}^{K}=\bigsqcup_{z \in Z} \mathcal{X}_{z}^{K}$ is a special subset of $\mathrm{R}\left(W^{(K)}\right)$ (see 39.1). Now the image of $\Omega^{K}$ under $\Omega \rightarrow \operatorname{Inn}(W)$ (as above) is contained in the group $\operatorname{Aut}\left(W^{(K)}, K, \mathcal{X}^{K}\right)$ of automorphisms of $W^{(K)}$ which preserve $K$ and $\mathcal{X}^{K}$. Thus we have a homomorphism $\Omega^{K} \rightarrow \operatorname{Aut}\left(W^{(K)}, K, \mathcal{X}^{K}\right)$. Restricting this to a subgroup $C$ of $\Omega^{K}$ we obtain a homomorphism $C \rightarrow \operatorname{Aut}\left(W^{(K)}, K, \mathcal{X}^{K}\right)$. Let $c \in \operatorname{Aut}(W, I, \mathcal{X})$ be such that $c(K)=K$. We have $c^{N}=1$ for some $N \geq 1$. We extend the bijection $c: I \stackrel{\sim}{\longrightarrow} I$ (restriction of $c: W \rightarrow W$ ) to a bijection $c: \tilde{I} \stackrel{\sim}{\longrightarrow} \tilde{I}$ by $c(\omega)=\omega$. We have $c\left(W^{(K)}\right)=W^{(K)}, c\left(\mathcal{X}^{K}\right)=\mathcal{X}^{K}$, hence $c$ restricts to an element of $\operatorname{Aut}\left(W^{(K)}, K, \mathcal{X}^{K}\right)$. For any $\sigma \in \Omega$ we define an element $c(\sigma) \in \Omega$ by $c(\sigma)(x)=\sigma\left(c^{-1} x\right)$ for $x \in \tilde{I}$. Then $c: \Omega \stackrel{\sim}{\longrightarrow} \Omega$ preserves $\Omega^{K}$. Assume that $c(C)=C$. For $\sigma \in C, w \in W^{(K)}$ we have $c(\sigma(w))=c(\sigma)(c(w))$. Let $\langle c\rangle$ be a cyclic group of order $N$ with generator $c$. On the set $W^{(K)} \times C \times\langle c\rangle$ we have 
a group structure $\left(w, \sigma, c^{n}\right)\left(w^{\prime}, \sigma^{\prime}, c^{n^{\prime}}\right)=\left(w \sigma\left(c^{n}\left(w^{\prime}\right)\right), \sigma c^{n}\left(\sigma^{\prime}\right), c^{n+n^{\prime}}\right)$. This is the semidirect product $W^{(K)} \Gamma$ of $W^{(K)}$ with the group $\Gamma=C \times\langle c\rangle$ with group structure $\left(\sigma, c^{n}\right)\left(\sigma^{\prime}, c^{n^{\prime}}\right)=\left(\sigma c^{n}\left(\sigma^{\prime}\right), c^{n+n^{\prime}}\right)$.

Proposition 39.7. In the setup of 39.6 let $E$ be a simple $\overline{\mathfrak{U}}\left[W^{(K)} \Gamma\right]$-module. Let $\gamma \in \Gamma$. There exists $\zeta$, a root of 1 in $\overline{\mathfrak{U}}$, such that $\operatorname{tr}(w \gamma, E) \in \zeta \mathbf{Z}$ for any $w \in W^{(K)}$.

From the theory of affine Weyl groups it is known that one of the following holds:

(i) $\Omega$ is cyclic and $c(\sigma)=\sigma$ for all $\sigma \in \Omega$,

(ii) $\Omega$ is cyclic and $c(\sigma)=\sigma^{-1}$ for all $\sigma \in \Omega$,

(iii) $\Omega \cong \mathbf{Z} / 2 \times \mathbf{Z} / 2$.

Moreover, $C \subset \Omega$ (compatibly with the action of $c$ ). In cases (i),(ii), $C$ is cyclic and the assumptions of Lemma 39.5 are satisfied (with $W^{(K)}$ instead of $W$ ). Hence the result follows from 39.5 .

In the remainder of the proof we assume that we are in case (iii). If $C$ is cyclic, then $c(\sigma)=\sigma=\sigma^{-1}$ for all $\sigma \in C$. The result follows again from Lemma 39.5. Hence we may assume that $C$ is not cyclic so that $C=\Omega$. Let $n$ be the order of $c: C \rightarrow C$. Then $n \in\{1,2,3\}$ and $n$ divides $N$. Note that $c^{N / n}$ is in the center of $\Gamma$. Tensoring $E$ by a suitable one-dimensional representation (which is trivial on $W^{(K)} C$ ) we may assume that $c^{N / n}$ acts trivially on $E$, hence $E$ factors through the quotient of $W^{(K)} \Gamma$ by the subgroup generated by $c^{N / n}$. Hence we may assume that $N=n$. If $n=1$, then $\Gamma \cong \mathbf{Z} / 2 \times \mathbf{Z} / 2$ is a group as in 39.5 and the result follows from 39.5. If $n=2$, then $\Gamma$ is a dihedral group of order 8 which is again a group as in 39.5 (an extension of $\mathbf{Z} / 2$ by $\mathbf{Z} / 4$ ) and the result follows from 39.5. If $n=3$ then $W$ must be of type $D_{4}, W^{(K)}$ is an elementary abelian 2-group, $E$ is one-dimensional and there exists a homomorphism $\mu: W^{(K)} \Gamma \rightarrow \overline{\mathfrak{U}}^{*}$ such that $\operatorname{tr}\left(w \gamma^{\prime}, E\right)=\mu\left(w \gamma^{\prime}\right)$ for all $w \in W^{(K)}, \gamma^{\prime} \in \Gamma$. Then $\zeta=\mu(\gamma)$ is a root of 1 . For any $w \in W^{(K)}$ we have $\mu(w \gamma)=\zeta \mu(w)$ and $\mu(w)= \pm 1$, since $w^{2}=1$. Thus, $\operatorname{tr}(w \gamma, E)= \pm \zeta$. The proposition is proved.

Corollary 39.8. Assume that $G, D, A, u$ are as in 35.22 and that $G^{0} / \mathcal{Z}_{G^{0}}$ is simple. Then $b_{A, u}^{v} \in \eta \mathbf{Q}$ for some $\eta$, a root of 1 (with $b_{A, u}^{v}$ as in 34.19.)

This follows from 35.22 and 39.7.

\section{REFERENCES}

[Al] D. Alvis, The duality operation in the character ring of a finite Chevalley group, Bull. Amer. Math. Soc. 1 (1979), 907-911. MR0546315 (81e:20012)

[BBD] A. Beilinson, J. Bernstein, P. Deligne, Faisceaux pervers, Astérisque 100 (1982). MR0751966 (86g:32015)

$[\mathrm{Cu}]$ C.W. Curtis, Truncation and duality in the character ring of a finite group of Lie type, J. Algebra 62 (1980), 320-332. MR0563231 (81e:20011)

[De] P. Deligne, La conjecture de Weil, II, Publ. Math. I.H.E.S. 52 (1980), 137-252. MR0601520 (83c:14017)

[Ka] N. Kawanaka, Fourier transforms of nilpotently supported invariant functions on a simple Lie algebra over a finite field, Invent. Math. 69 (1982), 411-435. MR0679766 (84c:20053)

[L14] G. Lusztig, Characters of reductive groups over a finite field, Ann. Math. Studies 107, Princeton Univ. Press, 1984. MR 0742472 (86j:20038)

[L3] G. Lusztig, Character sheaves, I, Adv. Math. 56 (1985), 193-237; II, vol. 57, 1985, pp. 226265; III, vol. 57, 1985, pp. 266-315; IV, vol. 59, 1986, pp. 1-63; V, vol. 61 , 1986, pp. 103155. MR0792706 (87b:20055) MR0806210 (87m:20118a) MR0825086 (87m:20118b) $\operatorname{MR} 0849848(87 \mathrm{~m}: 20118 \mathrm{c})$ 
[L9] G. Lusztig, Character sheaves on disconnected groups, I, Represent. Theory (electronic) 7 (2003), 374-403; II, vol. 8, 2004, pp. 72-124; III, vol. 8, 2004, pp. 125-144; IV, vol. 8, 2004, pp. 145-178; Errata , vol. 8, 2004, pp. 179-179; V, vol. 8, 2004, pp. 346-376; VI, vol. 8, 2004, pp. 377-413; VII, vol. 9, 2005, pp. 209-266. MR2017063(2006d:20090a) MR2048588 (2006d:20090b) MR2048589|(2006d:20090c) MR 2048590|(2006d:20090d) MR 2058725 MR2077486 (2005h:20111) MR:2084488 (2005h:20112) MR2133758(2006e:20089)

[L10] G. Lusztig, Parabolic character sheaves, I, Moscow Math. J. 4 (2004), 153-179. MR2074987 (2006d:20091a)

Department of Mathematics, Massachusetts Institute of Technology, Cambridge, MASSACHUSETTS 02139 